\title{
Difference Approximation of Nonlinear Evolution Equations and Semigroups of Nonlinear Operators
}

\author{
By \\ Nobuyuki KenMOCHI* and Sinnosuke OHARU**
}

\section{Introduction}

In this paper we introduce a notion of weak solution of a nonlinear differential equation

$$
(\mathrm{d} / \mathrm{dt}) u(t) \in A u(t), \quad 0<t<\tau
$$

in a Banach space $X$ and discuss the construction of weak solutions of the Cauchy problem for (DE) from the viewpoint of the difference approximation. The results obtained are applied to the generation problem of nonlinear semigroups.

We shall treat (DE) with the initial condition

$$
\lim _{t \rightarrow+0} u(t)=u_{0}
$$

and shall use the following approximating difference scheme:

$$
\begin{aligned}
& h_{p}^{-1}\left(u_{p, k}-u_{p, k-1}\right)-v_{p, k}=\varepsilon_{p, k}, \quad u_{p, 0}=u_{0} \\
& v_{p, k} \in A u_{p, k}, \quad k=1,2, \ldots, h_{p} \backslash 0 .
\end{aligned}
$$

The $h_{p}$ stands for the mesh size of the difference and $\varepsilon_{p, k}$ is the error which may occur at $k$-th step with mesh size $h_{p}$. If (DS) admits an approximate difference solution $\left\{u_{p, k} ; p, k=1,2, \ldots\right\}$ and if

Communicated by S. Matsuura, November 21, 1973.

* Department of Mathematics, Hiroshima University, Hiroshima.

** Department of Mathematics, Waseda University, Tokyo and Department of Mathematics, Georgetown University, Washington, D. C., U. S. A. 


$$
u(t)=\lim _{\substack{k h_{p} \rightarrow t \\ p \rightarrow \infty}} u_{p, k}, \quad 0<t<\tau
$$

holds, then the limit $u(t)$ gives a "solution" of the Cauchy problem for (DE).

Recently, Crandall and Liggett [6; Theorem I] proved that if $A$ is a dissipative operator in $X$ satisfying the "range condition"

$$
R(I-h A) \supset D(A) \text { for } 0<h<h_{0},
$$

where $D(A)$ and $R(I-h A)$ denote the domain of $A$ and the range of $I-h A$ respectively, then $T(t) u=\lim _{k h \rightarrow t, h \rightarrow 0}(I-h A)^{-k} u$ exists for $u \in D(A)$ and $t \geqq 0$. The limit operators $T(t), t \geqq 0$, admit continuous extensions $\bar{T}(t)$ onto $\overline{D(A)}$ and $\{\bar{T}(t) ; t \geqq 0\}$ forms a semigroup of nonlinear contractions on $\overline{D(A)}$. Crandall and Liggett's result states that $u_{p, k}=(I-$ $\left.h_{p} A\right)^{-k} u_{0}, \quad k=0,1,2, \ldots$, gives a unique solution of (DS) with $\varepsilon_{p, k}=0$ such that (CV) holds. That is, condition (R) can be regarded as a sufficient condition for (DS) to have an exact solution and their result gives an answer to our problem. Our first purpose is to weaken condition (R) to a condition which guarantees the existence of approximate difference solution with errors $\left\{\varepsilon_{p, k}\right\}$ satisfying a certain stability condition and then to derive sufficient conditions for (CV).

On the other hand, Miyadera [18] improved another result of Crandall-Liggett [6; Theorem II], and showed that if $u(t)=\bar{T}(t) u_{0}$ obtained as above is strongly differentiable almost everywhere on $[0, \infty)$, then its derivative satisfies $(\mathrm{d} / \mathrm{dt}) u(t) \in \bar{A} u(t)$ for almost all $t>0$. This fact suggests that $\bar{T}(t) u_{0}$ should be called a "solution" of the Cauchy problem for (DE). It is unfortune, however, that $u(t)=\bar{T}(t) u_{0}$ is not necessarily differentiable. Therefore, it is necessary to introduce an approximate notion of generalized solution of (DE). For instance, Kato [10], Webb [25] and others show that if $A$ is a particular type of dissipative operator of semilinear form, then the limit $u(t)=\bar{T}(t) u_{0}$ is a solution of an integral equation which is deduced from (DE) for the $A$. This kind of solution is called a mild solution. Crandall [7] and Oharu-Takahashi [22] treat accretive and dissipative operators which are associated with certain first order quasilinear equations and show that the semigroups determined 
by those operators give generalized solutions of Kružkov's type of the Cauchy problems for the equations. Moreover, Bénilan [2] introduced a notion of "integral solution" together with "pseudo-generator". Our second purpose is to introduce a notion of "weak solution" and then to investigate the relationship between the limit obtained by (CV) and the solution of the Cauchy problem for (DE). Bénilan proves that an integral solution is unique under a certain condition which is an extended form of the range condition ( $\mathrm{R})$. Our weak solution is an integral solution and is unique, under the assumption that $A-\omega$ be dissipative for some $\omega \geqq 0$. Moreover, we shall consider the relationship between the generalized domain $\hat{D}(A)$ of $A$ and the Lipschitz continuity of weak solutions of (DE).

The arguments mentioned above can be considered from the viewpoint of the theory of nonlinear semigroups. Our third purpose is to discuss the generation of a nonlinear semigroup associated with the given operator $A$.

This paper consists of five sections. Section 1 contains some special notations used in this paper, some basic notions and the fundamental facts concerning those notions. In Section 2, a notion of weak solution is introduced and a Cauchy problem in a weak sense for (DE) is formulated. Moreover, in that section, an approximating difference scheme for the Cauchy problem is introduced and our main results are given. Section 3 deals with the convergence of the difference approximation. In Section 4, the unicity of a weak solution is proved and some basic properties of weak solutions are studied. Finally, Section 5 treats the generation problem of semigroups of local type.

\section{§1. Preliminaries}

In this section we list some notations, basic notions and some of their fundamental properties.

Let $X$ be a real Banach space with norm $\|\cdot\|$. By an operator $A$ in $X$ we mean a (possibly multi-valued) operator with domain $D(A)$ and range $R(A)$ in $X$, that is, $A$ assigns to each $u \in X$ a subset $A u$ of 
$X . \quad D(A)$ is the set $\{u \in X ; A u \neq \emptyset\}$ and $R(A)=\bigcup_{u \in X} A u ; A u$ is the empty set if $u \notin D(A)$. A single-valued operator is referred to as a special case of a multi-valued operator in which $A u, u \in D(A)$, denotes the value of $A$ at $u$ or the singleton set consisting of this element.

By $G(A)$ we denote the graph of the operator $A$, i.e.,

$$
G(A)=\{(u, v) \in X \times X ; v \in A u, u \in D(A)\}
$$

We can identify the operator $A$ with its graph $G(A)$. For each $u \in D(A)$, we write $\|A u\|$ for $\inf \{\|v\| ; v \in A u\}$.

Let $S \subset X$. We write $A[S]$ for $\cup_{u \in S} A u$. By the restriction of $A$ to $S$, denoted by $A \mid S$, we mean an operator such that $D(A \mid S)=D(A) \cap S$ and $(A \mid S) u=A u$ for $u \in D(A) \cap S . \quad \bar{S}$ denotes the closure of $S$ in $X$. Let $A$ and $B$ be operators in $X . \quad B$ is called the closure of $A$ if $G(B)=$ $\overline{G(A)}$ in $X \times X$; we write $B=\bar{A}$. We say that $B$ is an extension of $A$, and $A$ is a restriction of $B$ (denoted by $A \subset B$ ), if $G(A) \subset G(B)$. For the notations of addition, scalar multiplication and composition of operators in $X$, we use the same notations as in Oharu [21]. We sometimes write $\gamma$ for the operator $\gamma I$, where $I$ denotes the identity operator on $X$ and $\gamma \in R$. For an operator $A, A^{-1}$ stands for the inverse of $A$ which is defined by

$$
G\left(A^{-1}\right)=\{(v, u) ;(u, v) \in G(A)\} .
$$

Moreover, given $z \in X, A+z$ denotes the operator defined by the correspondence $u \mapsto A u+z, u \in D(A)$.

Let $A$ be a single-valued operator in $X$ such that $R(A) \subset D(A)$. Then for positive integers $i$, we can define the iterations $A^{i}$ on $D(A)$ by $A^{i} u=$ $A\left(A^{i-1} u\right)$; we write $A^{0}=I$.

We denote by $\left\langle u, u^{*}\right\rangle$ the natural pairing between $u \in X$ and $u^{*} \in X^{*}$. By the duality map $F$ of $X$ we mean the (multi-valued) mapping from $X$ into its dual space $X^{*}$ defined by

$$
F u=\left\{u^{*} \in X^{*} ;\left\langle u, u^{*}>=\|u\|^{2}=\left\|u^{*}\right\|^{2}\right\}, \quad u \in X .\right.
$$

We define functionals $<,>_{i}: X \times X \rightarrow R^{1}$ and $<,>_{s}: X \times X \rightarrow R^{1}$ by 


$$
<u, v\rangle_{i}=\inf \left\{<u, v^{*}>; v^{*} \in F(v)\right\}
$$

and

$$
<u, v\rangle_{s}=\sup \left\{<u, v^{*}>; v^{*} \in F(v)\right\}
$$

respectively. These functionals have the following properties (CrandallLiggett [6; Lemma 2.16]):

Proposition 1.1. Let $u, v, w \in X$. Then we have:

(a) $\left.\langle u, v\rangle_{s}=-<-u, v\right\rangle_{i}$.

(b) $<\lambda v+u, v>_{j}=\lambda\|v\|^{2}+<u, v>_{j}$ for $\lambda \in R^{1}$ and $j=i$ or $s$.

(c) $<\lambda u, \mu v>_{j}=\lambda \mu<u, v>_{j} \quad$ for $\lambda \mu \geqq 0$ and $j=i$ or $s$.

(d) $\left\langle w+u, v>_{j} \leqq\|w\| \cdot\|v\|+\left\langle u, v>_{j}\right.\right.$ for $j=i$ or $s$.

(e) $\langle w+u, v\rangle_{s} \leqq\langle w, v\rangle_{s}+\langle u, v\rangle_{s}$ and $\langle w-u, v\rangle_{s} \geqq\langle w, v\rangle_{s}$ $-\langle u, v\rangle_{s}$.

(f) $<,>_{s}: X \times X \rightarrow R^{1}$ is upper semicontinuous with respect to the strong topology of $X \times X$.

(g) For any one $j$ of $s$ and $i$, there exists a $v^{*} \in F v$ such that $<u, v\rangle_{j}=\left\langle u, v^{*}>\right.$.

The canonical injection from $X$ into its bidual $X^{* *}$ is isometrically isomorphic, hence the duality mapping $F^{* *}$ of $X^{* *}$ is an extension of $F$. We denote by the same notations $<,>$ and $<,>_{j}, j=s, i$, the natural pairing between $X^{* *}$ and $X^{* * *}$ and the functionals on $X^{* *} \times$ $X^{* *}$ mentioned as above.

Let $C \subset X$ and let $T$ be a single-valued operator in $X . T$ is called a contraction on $C$ if $\left\|T u_{1}-T u_{2}\right\| \leqq\left\|u_{1}-u_{2}\right\|$ for $u_{1}, u_{2} \in C$. An operator $A$ in $X$ is said to be dissipative if

$$
<v_{1}-v_{2}, u_{1}-u_{2}>_{i} \leqq 0 \quad \text { for }\left(u_{1}, v_{1}\right),\left(u_{2}, v_{2}\right) \in G(A) .
$$

It is well-known (Kato $[11$; Lemma 1.1]) that $A$ is dissipative if and 
only if

$$
\begin{aligned}
& \left\|u_{1}-u_{2}\right\| \leqq\left\|\left(u_{1}-\lambda v_{1}\right)-\left(u_{2}-\lambda v_{2}\right)\right\| \\
& \qquad \text { for } \lambda>0 \text { and }\left(u_{i}, v_{i}\right) \in G(A) .
\end{aligned}
$$

Note that (1.2) implies that for every $\lambda>0,(I-\lambda A)^{-1}$ exists as a contraction on $R(I-\lambda A)$. If $A$ is a dissipative operator such that $R\left(I-\lambda_{0} A\right)=X$ for some $\lambda_{0}>0$, then we say that $A$ is $m$-dissipative. It is well-known that if $A$ is $m$-dissipative then $R(I-\lambda A)=X$ for $\lambda>0$. If $A$ is dissipative in $X$, then so is $\bar{A}$.

Let $S \subset X$ and $A$ be a dissipative operator in $X$. Then $A$ is said to be maximal dissipative on $S$ if any dissipative extension of $A$ coincides on $S$ with $A$.

Let $C \subset X$. A one-parameter family $\{T(t) ; t \geqq 0\}$ of operators from $C$ into itself is called a semigroup of local type on $C$ if it has the following properties:

(1.3) there is a constant $\omega \geqq 0$ such that $\mathrm{e}^{-\omega t} T(t)$ is a contraction on $C$ for $t \geqq 0$,

(1.4) $T(0)=I \mid C, T(t+s)=T(t) T(s) \quad$ for $t, s \geqq 0$,

(1.5) for each $u \in C, T(t) u$ is strongly continuous in $t \geqq 0$.

If $T$ is a Lipschitz continuous operator from $C$ into itself, then $\bar{T}$ is a Lipschitz continuous operator from $\bar{C}$ into itself. Hence, if $\{T(t) ; t \geqq 0\}$ is a semigroup of local type on $C$, then $\{\overline{T(t)} ; t \geqq 0\}$ forms a semigroup of local type on $\bar{C}$. On the generation of semigroups of local type, the following theorem due to Crandall-Liggett [6; Theorem I] is fundamental:

Theorem 1.2. Let $A-\omega$ be a dissipative operator in $X$ for some $\omega \geqq 0$. Let $A$ satisfy condition (R): $R(I-h A) \supset D(A)$ for $0<h<1 / \omega$. Then there exists a semigroup $\{T(t) ; t \geqq 0\}$ of local type on $D(A)$ such that

$$
T(t) u=\lim _{\substack{k h \rightarrow t \\ h \rightarrow 0}}(I-h A)^{-k} u \quad \text { for } t \geqq 0 \text { and } u \in D(A) .
$$


This theorem was proved in an ingenious way and the proof of our main theorem (which will be given in Section 3) is essentially due to it.

Finally, we prepare some estimates which play a central role in our arguement.

Proposition 1.3. Let $A-\omega$ be dissipative in $X$. Then we have:

(a) for $\left(u_{1}, v_{1}\right),\left(u_{2}, v_{2}\right) \in G(A)$ and for $\lambda$ with $0<\lambda<1 / \omega$,

$$
(1-\lambda \omega)\left\|u_{1}-u_{2}\right\| \leqq\left\|\left(u_{1}-\lambda v_{1}\right)-\left(u_{2}-\lambda v_{2}\right)\right\| ;
$$

(b) for $\left(u_{1}, v_{1}\right),\left(u_{2}, v_{2}\right) \in G(A)$ and for $\lambda, \mu$ with $0<\mu \leqq \lambda<1 / \omega$,

$$
\begin{array}{r}
(1-\mu \omega)\left\|u_{1}-u_{2}\right\| \leqq \\
(\mu / \lambda)\left\|\left(u_{1}-\mu v_{1}\right)-\left(u_{2}-\lambda v_{2}\right)\right\| \\
+(1-\mu / \lambda)\left\|\left(u_{1}-\mu v_{1}\right)-u_{2}\right\|
\end{array}
$$

Proof. (a) Let $\left(u_{1}, v_{1}\right),\left(u_{2}, v_{2}\right) \in G(A)$ and let $0<\lambda<1 / \omega$. Then

$$
\begin{aligned}
\left\|\left(u_{1}-\lambda v_{1}\right)-\left(u_{2}-\lambda v_{2}\right)\right\| \cdot\left\|u_{1}-u_{2}\right\| & \geqq<\left(u_{1}-\lambda v_{1}\right)-\left(u_{2}-\lambda v_{2}\right), f> \\
& \geqq(1-\lambda \omega)\left\|u_{1}-u_{2}\right\|^{2}
\end{aligned}
$$

for some $f \in F\left(u_{1}-u_{2}\right)$. From this (a) follows.

(b) Let $\left(u_{1}, v_{1}\right),\left(u_{2}, v_{2}\right) \in G(A)$ and let $0<\mu \leqq \lambda<1 / \omega$. Since $u_{2}-\mu v_{2}$ $=(\mu / \lambda)\left(u_{2}-\lambda v_{2}\right)+(1-\mu / \lambda) u_{2}$, (a) yields that

$$
\begin{aligned}
& (1-\mu \omega)\left\|u_{1}-u_{2}\right\| \leqq\left\|\left(u_{1}-\mu v_{1}\right)-\left(u_{2}-\mu v_{2}\right)\right\| \\
& \quad \leqq(\mu / \lambda)\left\|\left(u_{1}-\mu v_{1}\right)-\left(u_{2}-\lambda v_{2}\right)\right\|+(1-\mu / \lambda)\left\|\left(u_{1}-\mu v_{1}\right)-u_{2}\right\| .
\end{aligned}
$$

Q.E.D.

\section{§2. Difference Approximation of (DE)}

In this section we introduce a notion of weak solution of (DE) and formulate a Cauchy problem in a weak sense, WCP, for (DE). We then discuss the difference approximation to the WCP and state our main results along with some comments. Moreover, we shall list some 
examples of WCP's in which the relationship between the limit of difference approximation and the solution of WCP is studied.

Henceforth we let $A$ be an operator in $X$ such that $A-\omega$ is dissipative for some $\omega \geqq 0$. Let $J$ be a bounded interval whose end points are $a$ and $b, a<b$, and let us consider a differential equation in $X$

$$
(\mathrm{d} / \mathrm{dt}) u(t) \in A u(t) \quad \text { for } \quad t \in J
$$

For this (DE) Brezis and Pazy [4] have introduced the following type of solution:

Definition 2.1. An $X$-valued function $u(t)$ on $J$ is called a strong solution of (DE) on $J$ if it satisfies

(i) $u(t)$ is Lipschitz continuous on $J$,

(ii) $u(t)$ satisfies (DE) almost everywhere on $J$.

Condition (i) implies that $u(a)=\lim _{t \rightarrow a+0} u(t)$ and $u(b)=\lim _{t \rightarrow b-0} u(t)$ are defined. Hence, $u(t)$ can be regarded as a Lipschitz continuous function on $\bar{J}$ and its derivative is essentially bounded on $\bar{J}$. As was mentioned in the Introduction, the above notion of solution is too strong in the case of non-reflexive Banach spaces. We wish then to introduce other notions of solution. Let $u(t)$ be an $X$-valued function on a closed interval $\bar{J}=[a, b]$. Then we say that $u(t)$ satisfies condition (I) on $\bar{J}$, if the following condition holds:

(I) $u(t)$ is strongly continuous on $\bar{J}$ and there exists a null sequence $\left\{h_{p}\right\}$ of positive numbers and sequences $\left\{u_{p}(t)\right\}$ and $\left\{v_{p}(t)\right\}$ of $X$-valued, strongly measurable functions on $J$ such that

(i ) $u_{p}(t) \in D(A)$ and $v_{p}(t) \in A u_{p}(t)$ almost everywhere on $J$,

(ii) for every $\varepsilon>0, h_{p}^{-1}\left(u_{p}(t)-u_{p}\left(t-h_{p}\right)\right)-v_{p}(t) \rightarrow 0$ in $L^{1}\left(J_{\varepsilon} ; X\right)$ as $p \rightarrow \infty$, where $J_{\varepsilon}=[a+\varepsilon, b]$,

(iii) $u_{p}(t) \rightarrow u(t)$ in $L^{\infty}(J ; X)$ as $p \rightarrow \infty$.

A strong solution of (DE) on $\bar{J}=[a, b]$ satisfies condition (I) mentioned above. In fact, let $h_{p} \downarrow 0, u_{p}(t)=u(t)$ and let $v_{p}(t)=u^{\prime}(t), \quad p=$ 
$1,2,3, \ldots \quad$ Then, $\quad h_{p}^{-1}\left\|u_{p}(t)-u_{p}\left(t-h_{p}\right)\right\| \leqq M \quad$ for $\quad t \in \bar{J} \quad$ with $t>a+h_{p}$, where $M$ is the smallest Lipschitz constant of $u(t)$. Hence $\left\|v_{p}(t)\right\| \leqq M$ almost everywhere on $\bar{J}$ and the dominated convergence theorem yields that for every $\varepsilon>0, h_{p}^{-1}\left(u_{p}(t)-u_{p}\left(t-h_{p}\right)\right)-v_{p}(t) \rightarrow 0$ in $L^{1}\left(J_{\varepsilon} ; X\right)$ as $p \rightarrow \infty$, where $J_{\varepsilon}=[a+\varepsilon, b]$. Also, in a certain case, the function $u(t)$ satisfying condition (I) is closely related to the notion of generalized solution (in the sense of Oleinik) which is treated in the theory of first-order quasilinear equations. See Oharu-Takahashi [22; Section 6].

Condition (I) can be modified as follows: We say that an $X$-valued function $u(t)$ on a closed interval $\bar{J}=[a, b]$ satisfies condition (I) ${ }^{\prime}$ on $\bar{J}$, if it satisfies

(I) $u(t)$ is strongly continuous on $\bar{J}$ and there exists a family $\left\{J_{k} ; k=1,2, \ldots, N\right\}$ of a finite number of closed subintervals of $\bar{J}$ such that

(i) $J_{k}=\left[a_{k}, a_{k+1}\right]$ for $k=1,2, \ldots, N-1$, where $a_{1}=a$ and $a_{N}=b$,

(ii) $)_{s}^{\prime}$ for each $k, u(\cdot) \mid J_{k}$ satisfies condition (I) on the interval $J_{k}$.

Let $J$ be a (not necessarily closed) bounded interval with end points $a$ and $b$, and let $u(t)$ be an $X$-valued function on $J$. Then we say that $u(t)$ satisfies condition (II) on $J$, if the following condition is fulfilled:

(II) $u(t)$ is strongly continuous on $J$ and there exist a family $\left\{J_{k}^{p} ; k=1,2, \ldots, N_{p}, p=1,2,3, \ldots\right\}$ of closed subintervals of $J$ and a sequence $\left\{u_{p}\right\}$ of $X$-valued, strongly measurable functions on $J$ such that

(i ) $J_{k}^{p}=\left[a_{k}^{p}, b_{k}^{p}\right], b_{k}^{p} \leqq a_{k+1}^{p}$ for $k=1,2, \ldots, N_{p}$ and $p=1,2, \ldots$,

(ii) for every $p$ and $k, u_{p}(\cdot) \mid J_{k}^{p}$ satisfies condition (I) on the interval $J_{k}^{p}$,

(iii) $\sum_{k=0}^{N_{p}}\left(a_{k+1}^{p}-b_{k}^{p}\right) \rightarrow 0$ and $\sum_{k=1}^{N_{p}-1}\left\|u_{p}\left(a_{k+1}^{p}\right)-u_{p}\left(b_{k}^{p}\right)\right\| \rightarrow 0$ as $p \rightarrow \infty$, where $b_{0}^{p}=a$ and $a_{N_{p}+1}^{p}=b$,

(iv) $\sup _{t \in J^{p}}\left\|u_{p}(t)-u(t)\right\| \rightarrow 0$ as $p \rightarrow \infty$, where $J^{p}=\bigcup_{k} J_{k}^{p}$. 
Remark. Let $u_{1}(t)$ and $u_{2}(t)$ be $X$-valued functions satisfying condition (II) on intervals $[a, b)$ and $[b, c)$, respectively. If $u_{2}(b)=$ $\lim _{t \uparrow b} u_{1}(t)$, then the function $u(t)$ defined by

$$
u(t)=\left\{\begin{array}{lll}
u_{1}(t) & \text { for } & t \in[a, b) \\
u_{2}(t) & \text { for } & t \in[b, c)
\end{array}\right.
$$

satisfies condition (II) on $[a, c)$, that is, a continuation of $X$-valued functions satisfying condition (II) satisfies condition (II) on its domain.

Moreover, we shall see in Section 4 that an $X$-valued function $u(t)$ satisfying condition (II) on $J$ gives an integral solution which has recently been proposed by Bénilan [1]:

Definition 2.2. Let $u(t)$ be a continuous function on $J . u(t)$ is said to be an integral solution of (DE) on $J$, if for every pair $(s, t) \in$ $J \times J$ with $s \leqq t$,

$$
\mathrm{e}^{-2 \omega t}\|u(t)-x\|^{2}-\mathrm{e}^{-2 \omega s}\|u(s)-x\|^{2} \leqq 2 \int_{s}^{t} \mathrm{e}^{-2 \omega \tau}<y, u(\tau)-x>_{s} d \tau
$$

holds for all $(x, y) \in G(A)$.

We can obtain the following result (Theorem 4.4 in Section 4):

Theorem 2.3. For each $u_{0} \in \overline{D(A)}$, there exists at most one $X$ valued function satisfying condition (II) on $J$ and $\lim _{t \rightarrow a+0} u(t)=u_{0}$.

In view of this we introduce two types of solution of (DE):

Definition 2.4. An $X$-valued function $u(t)$ on a bounded closed interval $\bar{J}=[a, b]$ is called a simple weak solution of (DE) on $\bar{J}$ if it satisfies condition (I)' on $\bar{J}$.

Definition 2.5. An $X$-valued function $u(t)$ on $J$ is called a weak solution of (DE) on $J$ if it satisfies condition (II) on $J$.

By a Cauchy problem in a weak sense, WCP, formulated on $J$ for an operator $A$ we mean the following:

WCP. Given $u_{0}$, find a weak solution $u(t)$ of (DE) on $J$ satisfying 
the initial condition $\lim _{t \downarrow a} u(t)=u_{0}$.

The $u_{0}$ is called the initial-value and a weak solution $u(t)$ of (DE) on $J$ satisfying $\lim _{t \downarrow a} u(t)=u_{0}$ is called the solution of WCP associated with the initial-value $u_{0}$. The initial-value $u_{0}$ of a solution of WCP must belong to $\overline{D(A)}$ and Theorem 2.3 states that a solution of WCP associated with the initial-value $u_{0} \in \overline{D(A)}$ is unique.

By a Cauchy problem, CP, formulated on $J$ for $A$ we mean the following:

CP. Given $u_{0}$, find a strong solution $u(t)$ of (DE) on $J$ satisfying $\lim _{t \downarrow a} u(t)=u_{0}$.

A strong solution $u(t)$ of (DE) on $J$ satisfying $\lim _{t \downarrow a} u(t)=u_{0}$ is called a solution of $\mathrm{CP}$ associated with the initial-value $u_{0}$.

We want to find solutions of WCP by employing the finite-difference method. Here we consider the following type of difference approximation:

$$
\begin{aligned}
& h_{p}^{-1}\left(u_{p, k}-u_{p, k-1}\right)-v_{p, k}=\varepsilon_{p, k}, u_{p, 0}=u_{0}, \\
& v_{p, k} \in A u_{p, k}, k=1,2, \ldots, N_{p}=\left[(b-a) / h_{p}\right],
\end{aligned}
$$

where $\left\{h_{p}\right\}$ is a certain null sequence of positive numbers depending on $u_{0}$. The $\varepsilon_{p, k}$ is the error which may occur at the $k$-th step of the approximation with mesh size $h_{p}$. In this sense (DS) can be regarded as an approximating difference scheme for WCP which permits errors. For this type of difference scheme we introduce two notions of "approximate difference solution".

Definition 2.6. Let $v>0$. We call the family $\left\{\left(u_{p, k}^{v}, v_{p, k}^{v}\right)\right\} \subset G(A)$ $\left(\left\{u_{p, k}^{v}\right\} \subset D(A)\right.$ if $A$ is single-valued) an approximate difference solution of (DS) with error bound $v$ if there exists a $p_{0} \equiv p_{0}\left(u_{0}, J, v\right)$ such that $\left(\alpha_{1}\right)$ for every $p \geqq p_{0}$ and every $k$ with $1 \leqq k \leqq N_{p}=\left[(b-a) / h_{p}\right]$,

$$
h_{p}^{-1}\left(u_{p, k}^{v}-u_{p, k-1}^{v}\right)-v_{p, k}^{v}=\varepsilon_{p, k}^{v}, u_{p, 0}^{v}=u_{0}
$$

$\left(\alpha_{2}\right) \quad h_{p} \sum_{k=1}^{N_{p}}\left\|\varepsilon_{p, k}^{v}\right\| \leqq v$.

Definition 2.7. A family $\left\{\left(u_{p, k}, v_{p, k}\right)\right\} \subset G(A)\left(\left\{u_{p, k}\right\} \subset D(A)\right.$ if $A$ is 
single-valued) is called an asymptotically approximate difference solution of (DS), if

$$
\begin{aligned}
\left(\beta_{1}\right) \quad h_{p}^{-1}\left(u_{p, k}-u_{p, k-1}\right)-v_{p, k}=\varepsilon_{p, k}, \quad u_{p, 0} & =u_{0} \\
\text { for } 1 \leqq k \leqq N_{p}= & {\left[(b-a) / h_{p}\right] \text { and } p=1,2, \ldots, }
\end{aligned}
$$

$\left(\beta_{2}\right) \max _{1 \leqq k \leqq N_{p}}\left\|\varepsilon_{p, k}\right\| \rightarrow 0$ as $p \rightarrow \infty$.

If $\varepsilon_{p, k} \equiv 0$ for all $k$ and $p$, we say that $\left\{\left(u_{p, k}, v_{p, k}\right)\right\}$ is an exact difference solution of (DS).

An asymptotically approximate difference solution is an approximate difference solution with error bound $v$ for every $v>0$. In fact, let $\left\{\left(u_{p, k}, v_{p, k}\right)\right\} \subset G(A)$ be an asymptotically approximate difference solution of (DS). Let $v>0$ and choose a $p_{0}$ such that

$$
\max _{k}\left\|\varepsilon_{p, k}\right\| \leqq v /(b-a) \quad \text { for } p \geqq p_{0} \text {. }
$$

Then, $h_{p} \sum_{k=1}^{N_{p}}\left\|\varepsilon_{p, k}\right\| \leqq v$ for $p \geqq p_{0}$; this means that $\left\{\left(u_{p, k}, v_{p, k}\right)\right\}$ is an approximate difference solution with error bound $v$.

As will be seen in Examples 2.13, 2.14 and 2.15, the range conditions $(R)$ and $\left(R_{\text {loc }}\right)$ are special cases of the condition that (DS) has an approximate difference solution. On the convergence of the difference approximation, we have the following results:

Theorem 2.8. Let $u_{0} \in \overline{D(A)}$.

I. Assume that for every $v>0$, (DS) has an approximate difference solution $\left\{\left(u_{p, k}^{v}, v_{p, k}^{v}\right)\right\}$ with error bound $v$. Suppose that there is a constant $C>0$, independent of $v, p$ and $k$, such that

$$
\left\|u_{p, k}^{v}-u_{p, k-1}^{v}\right\| \leqq C\left(1+\left\|\varepsilon_{p, k}^{v}\right\|\right) h_{p}, \text { for } 1 \leqq k \leqq N_{p} \text { and } p \geqq p_{0} \text {, }
$$

where $p_{0}$ is the integer given in condition $\left(\alpha_{1}\right)$. Then:(a) there exists a unique simple weak solution $u(t)$ of (DE) on $\bar{J}=[a, b]$ such that for every sequence $v_{i} \downarrow 0$, there exists a subsequence $\left\{p_{i}\right\}$ and

$$
u(t)=\lim _{\substack{a+k h_{p_{i}} \rightarrow t \\ i \rightarrow \infty}} u_{p_{i}, k}^{v_{1}}, \quad t \in \bar{J}
$$


(b) $\quad\|u(t)-u(s)\| \leqq C \cdot|t-s|$ for $t, s \in \bar{J}$. (c) $u(a)=u_{0}$.

II. Assume that (DS) has an asymptotically approximate difference solution $\left\{\left(u_{p, k}, v_{p, k}\right)\right\}$ satisfying

$$
\left\|u_{p, k}-u_{p, k-1}\right\| \leqq C \cdot h_{p}, \quad k=1,2, \ldots, N_{p}, \quad p=1,2, \ldots
$$

Then we have:(a)' there exists a unique simple weak solution $u(t)$ of (DE) on $J$ such that

$$
u(t)=\lim _{\substack{a+m h_{p} \rightarrow t \\ p \rightarrow \infty}} u_{p, m}, \quad t \in \bar{J},
$$

together with (b) and (c).

The proof of this theorem will be given in the next two sections.

Remark 2.9. Suppose that (DS) admits an asymptotically approximate difference solution $\left\{\left(u_{p, k}, v_{p, k}\right)\right\}$ satisfying $\left(\beta_{2}\right)^{\prime}$ there exists a constant $M=M\left(u_{0}, J\right)>0$ such that

$$
\left\|\varepsilon_{p, k}\right\| \leqq M h_{p} \quad \text { for } k=1,2, \ldots, N_{p} \text { and } p=1,2, \ldots
$$

If there exists a constant $N>0$ such that $\left\|u_{p, 1}-u_{0}\right\| \leqq N h_{p}$ for $p \geqq 1$, then $(\mathrm{S})^{\prime}$ is satisfied. In fact, $\left\|u_{p, k}-u_{p, k-1}-h_{p} v_{p, k}\right\| \leqq M h_{p}^{2}$ for $1 \leqq k \leqq N_{p}$ and $p \geqq 1$; hence

$$
\begin{aligned}
\| u_{p, k}- & u_{p, k-1}\left\|\leqq \gamma_{p}\right\|\left(u_{p, k}-h_{p} v_{p, k}\right)-\left(u_{p, k-1}-h_{p} v_{p, k-1}\right) \| \\
= & \gamma_{p} \|\left\{u_{p, k}-h_{p} v_{p, k}-u_{p, k-1}\right\}+\left\{h_{p} v_{p, k-1}-u_{p, k-1}+u_{p, k-2}\right\} \\
& +\left\{u_{p, k-1}-u_{p, k-2}\right\} \| \\
\leqq & \gamma_{p}\left\|u_{p, k-1}-u_{p, k-2}\right\|+2 M \gamma_{p} h_{p}^{2} \leqq \gamma_{p}^{k}\left\|u_{p, 1}-u_{p, 0}\right\|+2 M \gamma_{p}^{k}(k-1) h_{p}^{2} \\
\leqq & \gamma_{p}^{k}\{N+2 M(b-a)\} h_{p}, \gamma_{p}=\left(1-h_{p} \omega\right)^{-1} .
\end{aligned}
$$

Theorem 2.8 states that if (DS) formulated for $u_{0} \in \overline{D(A)}$ admits an approximate difference solution satisfying condition (S), then it converges to a Lipschitz continuous function $u(t)$ on $\bar{J}$. Conditions (S) and $(S)^{\prime}$ are closely related to the generalized domain of $A$. The notion of generalized domain was first introduced by Crandall [8]; Bénilan [2] gives a modified version: 
Definition 2.10. Let $X^{* *}$ be the bidual of $X$. Since $D(A) \subset X \subset$ $X^{* *}, A$ can be regarded as an operator in $X^{* *}$. We define an extension $\hat{A}$ of $A$ whose graph is given by

$$
\begin{array}{r}
G(\hat{A})=\left\{(x, y) \in \overline{D(A)} \times X^{* *} ;<y-v, x-u>_{i} \leqq \omega\|u-x\|^{2}\right. \\
\qquad \text { for all }(u, v) \in G(A)\} .
\end{array}
$$

Here, note that $<,>_{i}$ is the functional on $X^{* *} \times X^{* *}$. We also consider the set

$$
\begin{array}{r}
\hat{D}(A)=\left\{x \in \overline{D(A)} ;{ }^{\exists}\left\{\left(x_{n}, y_{n}\right)\right\} \subset G(\widehat{A}): x_{n} \longrightarrow x \text { in } X\right. \\
\text { and } \left.\left\{y_{n}\right\} \text { is bounded in } X^{* *}\right\} .
\end{array}
$$

Clearly, $A \subset \hat{A}$ and $D(A) \subset D(\hat{A}) \subset \hat{D}(A) \subset \overline{D(A)}$. A dissipative operator $A$ in $X$, considered in $X^{* *}$ is still dissipative. Let $A-\omega$ be dissipative in $X$. Let $(x, y) \in G(\hat{A})$ and define an operator $A_{1}$ in $X^{* *}$ by $G\left(A_{1}\right)=G(A) \cup\{(x, y)\}$. Then $A_{1}-\omega$ is dissipative in $X^{* *}$. Moreover, we have the following (see Theorem 4.9):

Theorem 2.11. Let $u(t)$ be a weak solution of (DE) on an interval $J=[a, b)$. Then $u(a) \in \hat{D}(A)$ if and only if $u(t)$ is Lipschitz continuous on $J$.

The function $u(t)$ obtained by Theorem 2.8 was a solution of WCP for $A$. In order for it to be a solution of CP, some additional assumptions are needed:

Theorem 2.12. Let $A-\omega$ be maximal dissipative on $\overline{D(A)}$. Let $u_{0} \in \overline{D(A)}$. Then CP for $A$ and $u_{0}$ has a solution if and only if for every $v>0$, there exists an approximate difference solution of (DS) with error bound $v$ satisfying condition (S) and the limit $u(t)$ obtained by Theorem 2.8 is strongly differentiable almost everywhere on $J$.

Remark. If in the above theorem, condition (R) holds for $A$, then we obtain the convergence $u(t)=\lim _{h \rightarrow+0}(I-h A)^{-[t / h]} u_{0} ;$ in this case the maximal dissipativity of $A-\omega$ is not needed and it is sufficient to assume the closedness of $A$. See Brezis-Pazy [4] and Crandall-Liggett [6; Theorem II]. 
Now, in the remainder of this section we mention some examples of approximate difference solutions.

Example 2.13. Let $J=[0, \tau]$ and let us assume that $A$ satisfies condition (R). If $u_{0} \in D(A)$, then $\left\{\left(u_{p, k}, v_{p, k}\right)\right\}$ defined by

$$
\begin{gathered}
u_{p, k}=\left(I-h_{p} A\right)^{-k} u_{0}, \quad v_{p, k}=h_{p}^{-1}\left(u_{p, k}-u_{p, k-1}\right), \\
k=1,2, \ldots, N_{p}\left(=\left[\tau / h_{p}\right]\right) ; h_{p} \downarrow 0,
\end{gathered}
$$

is an exact difference solution of (DS). Moreover, in this case, condition (S)' with $C=\exp (2 \omega \tau)\left\|A u_{0}\right\| \|$ holds. In fact,

$$
\left\|u_{p, k}-u_{p, k-1}\right\| \leqq \gamma_{p}^{k-1}\left\|A u_{0}\right\| h_{p} \leqq h_{p} \exp (2 \omega \tau)\left\|A u_{0}\right\|
$$

for sufficiently large $p$, where $\gamma_{p}=\left(1-\omega h_{p}\right)^{-1}$.

As mentioned in the above example, condition ( $R$ ) yields that (DS) has an exact difference solution (2.1) for each $u_{0} \in D(A)$. Conversely, in order to check condition ( $R$ ) we must at least find the exact difference solution of (DS), whereas in our argument, it is sufficient to find just an approximate difference solution of (DS). It should be noted that we do not need to consider the "unicity" of the difference solution of (DS); this fact is important for extending the results obtained so far to more general cases. From this point of view, we can say that condition (R) is a special case of the assumption that (DS) has an asymptotically approximate difference solution satisfying condition (S)'.

Example 2.14. Let $A-\omega$ be dissipative. Let $C$ be a subset of $X$ such that $D(A) \subset C \subset \overline{D(A)}$ and let us assume the following condition which is an extended form of $(\mathrm{R})$ :

$\left(\mathrm{R}_{\text {loc }} ; A, C\right)$ For every $x \in C$, there exist a sequence $\left\{h_{p}\right\}$ of positive numbers, a sequence $\left\{z_{p}\right\}$ in $X$ and a neighbourhood $U$ of $x$ such that $h_{p} \rightarrow 0, z_{p} \rightarrow 0$ and

$$
\bigcap_{p} \overline{R\left(I-h_{p}\left(A+z_{p}\right)\right)} \supset C \cap U .
$$

Then for each $u_{0} \in D(\hat{A}) \cap C$, there exists an asymptotically approximate difference solution of (DS) satisfying (S)'. In fact, let $\left\{h_{p}\right\},\left\{z_{p}\right\}$ 
and $U=B\left(u_{0} ; \rho\right)$ be respectively the sequences and neighbourhood such that $\overline{R\left(I-h_{p}\left(A+z_{p}\right)\right)} \supset U \cap C$ for all $p$. We choose a positive number $\tau$ so that

$$
0<\tau<\rho /\left(2 \tau+\exp (2 \omega \tau)\left(\left\|v_{0}\right\|+\sup _{p}\left\|z_{p}\right\|\right)\right)
$$

where $v_{0} \in \hat{A} u_{0}$, and then construct $\left\{\left(u_{p, k}, v_{p, k}\right) ; k=0,1,2, \ldots, N_{p}=\left[\tau / h_{p}\right]\right.$, $p=1,2,3, \ldots\}$ as follows:

First, take an $\left(u_{p, 1}, v_{p, 1}\right) \in G(A)$ so that

$$
\left\|u_{p, 1}-h_{p} v_{p, 1}-h_{p} z_{p}-u_{0}\right\| \leqq \gamma_{p}^{-\left(N_{p}+1\right)} h_{p}^{2}, \quad \gamma_{p}=\left(1-\omega h_{p}\right)^{-1}
$$

and define an operator $A_{1}$ by $G\left(A_{1}\right)=G(A) \cup\left\{\left(u_{0}, v_{0}\right)\right\}$. Since $A_{1}-\omega$ is dissipative in $X^{* *}$, we see employing Proposition 1.3 that

$$
\begin{aligned}
\left\|u_{p, 1}-u_{0}\right\| & \leqq \gamma_{p}\left\|u_{p, 1}-h_{p} v_{p, 1}-u_{0}+h_{p} v_{0}\right\| \\
& \leqq \gamma_{p}^{1-\left(N_{p}+1\right)} h_{p}^{2}+\gamma_{p} h_{p}\left(\left\|v_{0}\right\|+\left\|z_{p}\right\|\right),
\end{aligned}
$$

and so, $u_{p, 1} \in D(A) \cap U$.

Now, assume that $\left(u_{p, j}, v_{p, j}\right) \in G(A), j=1,2, \ldots, k<N_{p}$, are defined such that $u_{p, j} \in D(A) \cap U$ and $\left\|u_{p, j}-h_{p} v_{p, j}-h_{p} z_{p}-u_{p, j-1}\right\| \leqq \gamma_{p}^{j-\left(N_{p}+1\right)} h_{p}^{2}$. We define $\left(u_{p, k+1}, v_{p, k+1}\right) \in G(A)$ as follows: By condition $\left(\mathrm{R}_{\mathrm{loc}} ; A, C\right)$ there exists an $\left(u_{p, k+1}, v_{p, k+1}\right) \in G(A)$ such that

$$
\left\|u_{p, k+1}-h_{p} v_{p, k+1}-h_{p} z_{p}-u_{p, k}\right\| \leqq \gamma_{p}^{k-N_{p}} h_{p}^{2} .
$$

Hence,

$$
\begin{aligned}
& \left\|u_{p, k+1}-u_{p, k}\right\| \leqq \gamma_{p}\left\|\left(u_{p, k+1}-h_{p} v_{p, k+1}\right)-\left(u_{p, k}-h_{p} v_{p, k}\right)\right\| \\
& =\gamma_{p} \|\left\{u_{p, k+1}-h_{p} v_{p, k+1}-h_{p} z_{p}-u_{p, k}\right\}+\left\{h_{p} v_{p, k}+h_{p} z_{p}-u_{p, k}+u_{p, k-1}\right\} \\
& \quad+\left\{u_{p, k}-u_{p, k-1}\right\} \| \\
& \leqq 2 \gamma_{p}^{k+1-N_{p}} h_{p}^{2}+\gamma_{p}\left\|u_{p, k}-u_{p, k-1}\right\| \\
& \leqq 2(k+1) \gamma_{p}^{k+1-N_{p}} h_{p}^{2}+\gamma_{p}^{k+1} h_{p}\left(\left\|z_{p}\right\|+\left\|v_{0}\right\|\right) \\
& \leqq \\
& h_{p}\left\{2 \tau+\left(\left\|v_{0}\right\|+\sup _{p}\left\|z_{p}\right\|\right) \exp (2 \omega \tau)\right\},
\end{aligned}
$$

and furthermore, 


$$
\left\|u_{p, k+1}-u_{0}\right\| \leqq \sum_{j=1}^{k+1}\left\|u_{p, j}-u_{p, j-1}\right\| \leqq \tau\left\{2 \tau+\exp (2 \omega \tau)\left(\left\|v_{0}\right\|+\sup _{p}\left\|z_{p}\right\|\right)\right\}<\rho .
$$

Thus, by induction, $\left\{\left(u_{p, k}, v_{p, k}\right) ; k=0,1,2, \ldots, N_{p}, p=1,2,3, \ldots\right\}$ is defined and simultaneously satisfies condition (S)' with $C=2 \tau+\left(\left\|v_{0}\right\|+\sup _{p}\left\|z_{p}\right\|\right)$ $\exp (2 \omega \tau)$. Moreover, since $\left\|\varepsilon_{p, k}\right\| \leqq \gamma_{p}^{k-N_{p}} h_{p}+\left\|z_{p}\right\| \leqq h_{p}+\left\|z_{p}\right\|$ for ${ }^{p} k=1$, $2, \ldots, N_{p}$, we see that $\max _{1 \leqq k \leqq N}\left\|\varepsilon_{p, k}\right\| \rightarrow 0$ as $p \rightarrow \infty$. Therefore, this system gives an asymptotically approximate difference solution of (DS). Consequently, it follows from Theorem 2.8-II that for each $u_{0} \in D(\widehat{A}) \cap C$ there is a unique (local) simple weak solution of (DE) on $[0, \tau]$ with the initial-value $u_{0}$.

Example 2.15. Let $C$ be a subset of $X$ such that $D(\widehat{A}) \subset C \subset \overline{D(A)}$ and $u_{0} \in C \cap \hat{D}(A)$. Assume condition $\left(\mathrm{R}_{\text {loc }} ; A, C\right)$. Then for every $v>0$, there exists an approximate difference solution of (DS) with error bound $v$ for which (S) holds. In fact, let $\left\{h_{p}\right\},\left\{z_{p}\right\}$ and $U=B\left(u_{0} ; \rho\right)$ be respectively the sequences and open ball such that $h_{p} \rightarrow 0, z_{p} \rightarrow 0$ and $\cap \overline{R\left(I-h_{p}\left(A+z_{p}\right)\right)} \supset C \cap U$. On the other hand, from the definition of $\stackrel{p}{\hat{D}}(A)$ one can find a positive constant $M$ such that for each $v>0$, there is an element $\left(x_{v}, x_{v}^{\prime}\right) \in G(\hat{A})$ with $\left\|x_{v}-u_{0}\right\| \leqq v$ and $\left\|x_{v}^{\prime}\right\| \leqq M$. For these $M$ and $\rho$ we choose a number $\tau$ so that

$$
0<\tau<\rho / 2\left\{2 \tau+\exp (2 \omega \tau)\left(M+\sup _{p}\left\|z_{p}\right\|\right)\right\} .
$$

Now, let $1>v>0, h_{p}+\left\|z_{p}\right\| \leqq v / 2 \tau$ and take an element $\left(x_{v}, x_{v}^{\prime}\right) \in G(\hat{A})$ such that $\left\|x_{v}-u_{0}\right\| \leqq \min \{v / 2, \rho / 2\}$ and $\left\|x_{v}^{\prime}\right\| \leqq M$. Then

$$
\bigcap_{p} \overline{R\left(I-h_{p}\left(A+z_{p}\right)\right)} \supset C \cap B\left(x_{v} ; \rho / 2\right) .
$$

Hence, as was seen in Example 2.14, there is an asymptotically approximate difference solution $\left\{\left(\tilde{u}_{p, k}, \tilde{v}_{p, k}\right)\right\}(\subset G(A))$ of (DS) such that $\tilde{u}_{p, 0}=x_{v}$ and $\left\|\tilde{\varepsilon}_{p, k}\right\|=\left\|h_{p}^{-1}\left(\tilde{u}_{p, k}-\tilde{u}_{p, k-1}\right)-\tilde{v}_{p, k}\right\| \leqq h_{p}+\left\|z_{p}\right\|$ for $k=1,2, \ldots, N_{p}\left(=\left[\tau / h_{p}\right]\right)$. Put $\left(u_{p, k}^{v}, v_{p, k}^{v}\right)=\left(\tilde{u}_{p, k}, \tilde{v}_{p, k}\right)$ for $k=1,2, \ldots, N_{p}$. Then,

$$
\begin{aligned}
\left\|\varepsilon_{p, 1}^{v}\right\| & \leqq\left\|h_{p}^{-1}\left(u_{p, 1}^{v}-x_{v}\right)-v_{p, 1}^{v}\right\|+\left\|h_{p}^{-1}\left(u_{0}-x_{v}\right)\right\| \\
& \leqq h_{p}+\left\|z_{p}\right\|+v / 2 h_{p}
\end{aligned}
$$

and 


$$
\left\|\varepsilon_{p, k}^{v}\right\| \leqq h_{p}+\left\|z_{p}\right\| \quad \text { for } k=2,3, \ldots, N_{p}
$$

hence

$$
h_{p} \sum_{k=1}^{N_{p}}\left\|\varepsilon_{p, k}^{v}\right\| \leqq v / 2+\sum_{k=1}^{N_{p}} h_{p}\left(h_{p}+\left\|z_{p}\right\|\right) \leqq \nu
$$

Moreover, as we have shown in Example 2.14, we have

$$
\left\|u_{p, k}^{v}-u_{p, k-1}^{v}\right\| \leqq h_{p}\left\{2 \tau+\left(M+\sup _{p}\left\|z_{p}\right\|\right) \exp (2 \omega \tau)\right\}
$$

for $2 \leqq k \leqq N_{p}$ and noting that $\left\|\varepsilon_{p, 1}^{v}\right\| \geqq h_{p}^{-1}\left\|x_{v}-u_{0}\right\|-v / 2 \tau$,

$$
\begin{aligned}
\left\|u_{p, 1}^{v}-u_{0}\right\| & \leqq\left\|u_{p, 1}^{v}-x_{v}\right\|+\left\|x_{v}-u_{0}\right\| \\
& \leqq h_{p}\left\{2 \tau+\left(M+\sup _{p}\left\|z_{p}\right\|\right) \exp (2 \omega \tau)\right\}+h_{p}\left\|\varepsilon_{p, 1}^{v}\right\|+v h_{p} / 2 \tau \\
& \leqq h_{p}\left\{2 \tau+1 / 2 \tau+\left(M+\sup _{p}\left\|z_{p}\right\|\right) \exp (2 \omega \tau)+\left\|\varepsilon_{p, 1}^{v}\right\|\right\} .
\end{aligned}
$$

Thus, $\left\{\left(u_{p, k}^{v}, v_{p, k}^{v}\right) ; k=0,1,2, \ldots, N_{p}, p=1,2, \ldots\right\}$ is defined and is an approximate difference solution of (DS) satisfying condition (S) with $C=2 \tau+1 / 2 \tau+\left(M+\sup _{p}\left\|z_{p}\right\|\right) \exp (2 \omega \tau)$. Consequently, it follows from Theorem 2.8-I that for each $u_{0} \in \hat{D}(A) \cap C$, there is a unique local simple weak solution of $(\mathrm{DE})$ on $[0, \tau]$ with the initial-value $u(0)=u_{0}$. In fact, we can continue this local solution to obtain a weak solution in a global sense; we shall mention the continuation in Section 4.

Example 2.16. Recently, Kružkov [16] discussed the problem of unicity and existence of a solution of the Cauchy problem for the following quasilinear equation:

$$
u_{t}+\sum_{i=1}^{d} \phi_{i}(u)_{x_{i}}=\psi(u) \quad t>0, x=\left(x_{1}, x_{2}, \ldots, x_{d}\right) \in R^{d} .
$$

Crandall [7] has treated this problem from the viewpoint of the theory of nonlinear semigroups by restricting $\psi(u) \equiv 0$.

Definition 2.17. Let $A_{0}$ be an operator defined in $X=L^{1}\left(R^{d}\right)$ by the following condition: $(u, v) \in G\left(A_{0}\right)$ if and only if $u, v \in X, \phi_{i}(u) \in X$ for $i=1,2, \ldots, d$ and for every nonnegative $f \in C_{0}^{\infty}\left(R^{d}\right)$ and every $k \in R^{1}$, 


$$
\int_{R^{d}} \operatorname{sign}(u(x)-k)\left\{\sum_{i=1}^{d}\left[\phi_{i}(u(x))-\phi_{i}(k)\right] f_{x_{i}}(x)-v(x) f(x)\right\} d x \geqq 0,
$$

where $\operatorname{sign} r$ is a function on $R^{1}$ such that $\operatorname{sign} r=1$ for $r>0,=0$ for $r=0$ and $=-1$ for $r<0$.

$A_{0}$ should be regarded as a multi-valued operator in general. If $u \in D\left(A_{0}\right) \cap L^{\infty}\left(R^{d}\right)$, then $A_{0} u=-\sum_{i=1}^{d} \phi_{i}(u)_{x_{i}}$ holds in the sense of distributions. Crandall proved that if $\phi_{i}$ is of class $C^{1}\left(R^{1}\right)$ and $\phi_{i}(0)=0$, then $\bar{A}_{0}$ is $m$-dissipative in $X$. Therefore, a semigroup $\{\bar{T}(t) ; t \geqq 0\}$ of nonlinear contractions on $X$ can be constructed by Theorem 1.2. Moreover, if $u_{0} \in X \cap L^{\infty}\left(R^{d}\right)$, then $u(t ; x)=\left[\bar{T}(t) u_{0}\right](x)$ gives a generalized solution in the sense that conditions (G.1), (G.2) and (G.3) (mentioned in Theorem 2.21) hold with $\psi \equiv 0$ and $M=0$.

Oharu and Takahashi [22] obtained the following result by employing a convergence theorem and an approximation theorem for nonlinear semigroups which are given in Brezis-Pazy [5] and MiyaderaOharu [20].

Let $X_{m}=\left\{u \in X \cap L^{\infty}\left(R^{d}\right) ;\|u\|_{\infty} \leqq m\right\}, \quad m=1,2, \ldots \quad$ and $\quad X_{0}=\bigcup_{m \geqq 1} X_{m}=$ $X \cap L^{\infty}\left(R^{d}\right)$.

Theorem 2.18. (i) There exists a single-valued, dissipative operator $A$ with domain $D(A)$ such that $C_{0}^{1}\left(R^{d}\right) \subset D(A) \subset X_{0}$ and for each $u \in D(A)$, $A u=-\sum_{i=1}^{d} \phi_{i}(u)_{x_{i}}$ holds in the sense of distributions.

(ii) For every $h>0, R(I-h A)=X_{0}(\supset D(A))$ and $v=(I-h A)^{-1} u$ satisfies

$$
v+h \sum_{i=1}^{d} \phi_{i}(v)_{x_{i}}=u
$$

(iii) For every $u_{0} \in X_{0}, T(t) u_{0}=\lim _{n \rightarrow \infty}\left(I-\frac{t}{n} A\right)^{-n} u_{0}$ holds for $t \geqq 0$ and the limit operators $T(t) ; t \geqq 0$, form an $L^{1}$-contractive semigroup on $X_{0}$. Moreover, $u(t, x)=\left[T(t) u_{0}\right](x)$ gives a generalized solution of (2.2) with $\psi \equiv 0$ in the sense that conditions (G.1)-(G.3) with $\psi \equiv 0$ and $M=0$ are satisfied.

Remark 2.19. It is proved that $A=A_{0} \mid D(A), D(A)=\left\{u \in D\left(A_{0}\right)\right.$; $\left.u, A_{0} u \in L^{\infty}\left(R^{d}\right)\right\}$. Hence $\bar{A}=\bar{A}_{0}$ and $\bar{A}$ is $m$-dissipative in $X$. Each 
$T(t)$ admits a continuous extension $\bar{T}(t)$ onto $X$ and $\{\bar{T}(t) ; t \geqq 0\}$ coincides with Crandall's semigroup.

If $u_{0} \in X_{0}$, then $T(t) u_{0}$ is a simple weak solution of (DE) for $A$ on every bounded closed subinterval of $[0, \infty)$. Moreover, as is seen from Theorems 4.7 and 4.10 , for each $u_{0} \in X, \bar{T}(t) u_{0}$ gives a unique weak solution of (DE) for $A$ on every bounded subinterval of $[0, \infty)$. This means that the Cauchy problem for (2.2) with $\psi \equiv 0$ has "solutions" for "bad initial data".

Now, let us consider the original equation (2.2). We assume that $\psi=\psi(\xi)$ is a locally Lipschitz continuous function on $R^{1}$ such that

$$
|\psi(\xi)| \leqq M|\xi| \quad \text { for } \quad \xi \in R^{1}
$$

Define an operator $\Psi$ on $X_{0}$ by $[\Psi u](x)=\psi(u(x)), x \in R^{d}$. Then by (2.3), $\Psi$ maps $X_{0}$ into itself;

$$
\|\Psi u\|_{q} \leqq M\|u\|_{q} \quad \text { for } u \in X_{0} \text { and } q=1, \infty,
$$

where $\|u\|_{1}$ and $\left\|u_{\infty}\right\|$ mean $L^{1-}$ and $L^{\infty}$-norm of $u$, respectively. Let $\omega_{m}$ be the smallest Lipschitz constant of $\psi$ on $[-m, m]$ and let $u$, $v \in X_{m}$. Then, since $|\psi(u(x))-\psi(v(x))| \leqq \omega_{m}|u(x)-v(x)|$ almost everywhere on $R^{d}$, we have

$$
\|\Psi u-\Psi v\|_{1} \leqq \omega_{m}\|u-v\|_{1} .
$$

Setting $A_{1}=A+\Psi, D\left(A_{1}\right)=D(A)$, we consider (DE) for $A_{1}$ :

$$
(\mathrm{d} / \mathrm{dt}) u(t)=A_{1} u(t), \quad t>0 ; u(0)=u_{0} .
$$

This is an abstract version in $X$ of (2.2). We then consider (DS) for this (2.6) and demonstrate that the system $\left\{u_{p, k}\right\}$ defined by

$$
\begin{aligned}
& u_{p, k}=\left(I-h_{p} A\right)^{-1}\left(I+h_{p} \Psi\right) u_{p, k-1}, k=1,2, \ldots, \\
& u_{p, 0}=u_{0} \in D\left(A_{1}\right), \quad h_{p} \downarrow 0,
\end{aligned}
$$

gives an asymptotically approximate difference solution of (DS).

Lemma 2.20. Let $\tau>0$. Then $\left\|u_{p, k}\right\|_{q} \leqq e^{M \tau}\left\|u_{0}\right\|$ for $0 \leqq k h_{p} \leqq \tau$ and $q=1, \infty$. 
Proof. First, it is seen from the proof of Theorem 2.18 that $\|(I-$ $\left.h_{p} A\right)^{-1} u\left\|_{q} \leqq\right\| u \|_{q}$ for $u \in X_{0}$ and $q=1, \infty$. Hence, (2.4) yields that $\left\|u_{p, 1}\right\|_{q} \leqq\left(1+h_{p} M\right)\left\|u_{0}\right\|_{q}$ for $q=1, \infty$; and so, $u_{p, 1} \in X_{0}$ and $\left\|u_{p, 1}\right\|_{q} \leqq$ $e^{M \tau}\left\|u_{0}\right\|_{q}$ for $q=1, \infty$. Next, suppose that $u_{p, k-1} \in X_{0}$ and $\left\|u_{p, k-1}\right\|_{q} \leqq$ $\left(1+h_{p} M\right)^{k-1}\left\|u_{0}\right\|_{q}$ for $q=1, \infty$. Then by (2.4) and (2.7), we have

$$
\left\|u_{p, k}\right\|_{q} \leqq\left\|\left(I+h_{p} \Psi\right) u_{p, k-1}\right\|_{q} \leqq\left(1+h_{p} M\right)^{k}\left\|u_{0}\right\|_{q} \leqq \mathrm{e}^{M \tau}\left\|u_{0}\right\|_{q} \quad \text { for } q=1, \infty \text {. }
$$

Let $\tau>0,\left\|u_{0}\right\| \leqq m_{0}, m \geqq \mathrm{e}^{M \tau} m_{0}$, and let $0 \leqq k h_{p} \leqq \tau$. Then $\left\{u_{p, k}\right\} \subset X_{m}$ by Lemma 2.20 and hence (2.5) yields that

$$
\begin{aligned}
& \left\|u_{p, k}-u_{p, k-1}\right\|_{1}=\left\|\left(I-h_{p} A\right)^{-1}\left(I+h_{p} \Psi\right) u_{p, k-1}-u_{p, k-1}\right\|_{1} \\
& \quad \leqq\left\|\left(I+h_{p} \Psi\right) u_{p, k-1}-\left(I+h_{p} \Psi\right) u_{p, k-2}\right\|_{1} \leqq\left(1+h_{p} \omega_{m}\right)\left\|u_{p, k-1}-u_{p, k-2}\right\|_{1} \\
& \quad \leqq\left(1+h_{p} \omega_{m}\right)^{k-1}\left\|u_{p, 1}-u_{p, 0}\right\|_{1} \leqq h_{p}\left(1+h_{p} \omega_{m}\right)^{k-1}\left\|A_{1} u_{0}\right\|_{1} .
\end{aligned}
$$

This means that $\left\{u_{p, k}\right\}$ satisfies condition (S) with $C=\exp \left(\omega_{m} \tau\right)\left\|A_{1} u_{0}\right\|_{1}$. Furthermore, since

$$
\begin{aligned}
u_{p, k}-u_{p, k-1}-h_{p} A_{1} u_{p, k} & =\left(I-h_{p} A\right) u_{p, k}-u_{p, k-1}-h_{p} \Psi u_{p, k} \\
& =h_{p}\left(\Psi u_{p, k-1}-\Psi u_{p, k}\right)
\end{aligned}
$$

(2.5) and (2.8) imply that

$$
\left\|u_{p, k}-u_{p, k-1}-h_{p} A_{1} u_{p, k}\right\|_{1} \leqq h_{p}^{2} \omega_{m} \exp \left(\omega_{m} \tau\right)\left\|A_{1} u_{0}\right\|_{1} .
$$

This means that $\left\{u_{p, k}\right\}$ satisfies conditions $\left(\beta_{1}\right)$ and $\left(\beta_{2}\right)$ and consequently, $\left\{u_{p, k}\right\}$ becomes an asymptotically approximate difference solution of (DS). Therefore by Theorem 2.8-II and a way similar to [22; Theorem 5.1], we obtain

Theorem 2.21. For every $u_{0} \in X_{0}$,

$$
u(t)=\lim _{\substack{k h_{p} \rightarrow t \\ p \rightarrow \infty}}\left[\left(I-h_{p} A\right)^{-1}\left(I+h_{p} \Psi\right)\right]^{k} u_{0}
$$

exists for $t \geqq 0$. $u(t)$ gives a unique weak solution of (2.6) and at the same time, $u(t, x)=[u(t)](x)$ is a unique generalized solution of (2.2) in the following sense: 
(G.1) for every $\tau>0,\|u(t)\|_{q} \leqq e^{M \tau}\left\|u_{0}\right\|_{q}$ for $t \in[0, \tau]$ and $q=1, \infty$,

(G.2) for every $k \in R^{1}$ and every nonnegative $f \in C_{0}^{\infty}\left((0, \infty) \times R^{1}\right)$,

$$
\begin{gathered}
\int_{0}^{\infty} \int_{R^{d}}\left\{|u(t, x)-k| f_{t}(t, x)+\operatorname{sign}(u(t, x)-k) \sum_{i=1}^{d}\left[\phi_{i}(u(t, x))-\phi_{i}(k)\right] f_{x_{i}}(t, x)\right. \\
+\operatorname{sign}(u(t, x)-k) \psi(u(t, x)) f(t, x)\} \mathrm{dxdt} \geqq 0,
\end{gathered}
$$

(G.3) $u(t)$ is strongly continuous in $t \geqq 0$ and $\lim _{t \rightarrow+0}\left\|u(t)-u_{0}\right\|_{1}=0$.

Example 2.22. Let $X$ be a space consisting of real-valued, uniformly continuous and bounded functions on $R^{1}$. $X$ is a Banach space under the supremum norm. Any real number $\alpha$ can be regarded as a constant function $\alpha(x) \equiv \alpha$ which belongs to $X$. We can introduce into $X$ a partial ordering “ $\geqq$ " by letting $u \geqq v$ if $u(x) \geqq v(x)$ for all $x \in R^{1}$; we write $u>v$ if $u \geqq v$ and $u \neq v$. We denote by $X_{0}^{+}$the positive cone, $\{u \in X$; $u>0\}$, of this partially ordered Banach space $X$. Now, let us consider a linear operator $\Delta$ defined by

$$
(\Delta u)(x)=u^{\prime \prime}(x), x \in R^{1} \quad \text { for } u \in D(\Delta)=\left\{u \in X ; u, u^{\prime} \in C^{1}\left(R^{1}\right), u^{\prime \prime} \in X\right\}
$$

$\Delta$ is the infinitesimal generator of a linear contraction semigroup $\left\{\mathrm{e}^{t \Delta}\right.$; $t \geqq 0\}$ defined by $\left[\mathrm{e}^{t \Delta} u\right](x)=(4 \pi t)^{-1 / 2} \int_{R^{1}} \exp \left\{-(x-\sigma)^{2} / 4 t\right\} u(\sigma) \mathrm{d} \sigma$. Also, every positive number $\lambda$ belongs to the resolvent set $\rho(\Delta)$ and (I$\lambda \Delta)^{-1}$ is given by $\left[(I-\lambda . \Delta)^{-1} u\right](x)=(4 \lambda)^{-1 / 2} \int_{R^{1}} \exp \{-|x-\sigma| / \sqrt{\lambda}\} u(\sigma) \mathrm{d} \sigma$. Observe that $(I-\lambda \Delta)^{-1} \alpha=\alpha$ for all $\alpha \in R^{1}$ and the operator $(I-\lambda \Delta)^{-1}$ is order-preserving. Next, we define an operator $R$ on $X_{0}^{+}$by

$$
[R u](x)=(u(x))^{1 / 2}, \quad x \in R^{1} .
$$

$R$ is also order-preserving. Since $\|R u-R v\| \leqq\|u-v\| / 2 \sqrt{\varepsilon}$ for $u, v \geqq$ $\varepsilon>0, R$ is Lipschitz continuous on $X_{\varepsilon}^{+}=\left\{u \in X^{+} ; u \geqq \varepsilon\right\}, \varepsilon>0$.

Now, we define an operator $A$ in $X$ by the relation

$$
A u=\Delta u+R u, \quad u \in X_{0}^{+} \cap D(\Delta),
$$

and consider the Cauchy problem for (DE):

$$
(\mathrm{d} / \mathrm{dt}) u(t)=A u(t), \quad t \geqq 0 ; \quad u(0)=u_{0},
$$


which is an abstract version in $X$ of the Cauchy problem for the semilinear equation $u_{t}=\Delta u+\sqrt{u}$. (For a detailed argument on this kind of problem, see Fujita-Watanabe [9])

Let $u_{0} \in D(A) \cap X^{+}$for an $\varepsilon>0$. We consider (DS) for this (2.12). We shall show that the system $\left\{u_{p, k}\right\}$ defined by

$$
\begin{gathered}
u_{p, k}=\left(I-h_{p} \Delta\right)^{-1}\left(I+h_{p} R\right) u_{p, k-1}, \quad u_{p, 0}=u_{0}, \\
k, p=1,2, \ldots
\end{gathered}
$$

gives an asymptotically approximate difference solution of (DS).

First, $\left(I+h_{p} R\right) u_{p, 0} \geqq\left(I+h_{p} R\right) \varepsilon=\varepsilon+h_{p} \sqrt{\varepsilon}$, and so,

$$
u_{p, 1}=\left(I-h_{p} \Delta\right)^{-1}\left(I+h_{p} R\right) u_{0} \geqq\left(I-h_{p} \Delta\right)^{-1}\left(I+h_{p} R\right) \varepsilon=\left(I+h_{p} R\right) \varepsilon .
$$

Similarly, $u_{p, 2} \geqq\left(I+h_{p} R\right)^{2} \varepsilon$. Inductively, we obtain

$$
u_{p, k} \geqq\left(I+h_{p} R\right)^{k} \varepsilon \geqq \varepsilon \quad \text { and } \quad\left\{u_{p, k}\right\} \subset X_{\varepsilon}^{+} .
$$

Next, since $\left\|u_{p, 1}-u_{p, 0}\right\| \leqq h_{p}\left\|A u_{0}\right\|$, we have

$$
\begin{aligned}
\left\|u_{p, k}-u_{p, k-1}\right\| & \leqq \\
& \left.=\|\left(I+h_{p} R\right) u_{p, k-1} R\right) u_{p, k-1}-\left(I-h_{p} \Delta\right) u_{p, k-1} \| \\
& \leqq\left(1+h_{p} / 2 \sqrt{\varepsilon}\right)^{k-1}\left\|u_{p, 1}-u_{p, 0}\right\| \leqq h_{p} \exp \left(k h_{p} / 2 \sqrt{\varepsilon}\right)\left\|A u_{0}\right\| .
\end{aligned}
$$

Therefore,

$$
\begin{aligned}
& \left\|u_{p, k}-h_{p} \Delta u_{p k}-h_{p} R u_{p, k}-u_{p, k-1}\right\| \\
& \quad=\left\|\left(I+h_{p} R\right) u_{p, k-1}-h_{p} R u_{p, k}-u_{p, k-1}\right\| \\
& \quad \leqq\left(h_{p} / 2 \sqrt{\varepsilon}\right)\left\|u_{p, k}-u_{p, k-1}\right\| \\
& \quad \leqq h_{p}^{2} \exp \left(k h_{p} / 2 \sqrt{\varepsilon}\right)\left\|A u_{0}\right\| / 2 \sqrt{\varepsilon} .
\end{aligned}
$$

Now, let $\tau>0$ and $C=C(\tau)=\left\|A u_{0}\right\| \exp (\tau / 2 \sqrt{\varepsilon})$. Then we have

$$
\begin{aligned}
& \left\|u_{p, k}-u_{p, k-1}\right\| \leqq C h_{p} \\
& \left\|u_{p, k}-h_{p} A u_{p, k}-u_{p, k-1}\right\| \leqq(C / 2 \sqrt{\varepsilon}) h_{p}^{2}, \quad k=1,2, \ldots, N_{p}=\left[\tau / h_{p}\right] .
\end{aligned}
$$


This means that $\left\{u_{p, k} ; k=1,2, \ldots, N_{p} ; p=1,2, \ldots\right\}$ gives an asymptotically approximate difference solution of (DS) satisfying (S)'. Since $\tau$ was arbitrary, Theorem 2.8-II yields the convergence

$$
u\left(t ; u_{0}\right)=\lim _{\substack{k h_{p} \rightarrow t \\ p \rightarrow \infty}} u_{p, k}=\lim _{\substack{k h_{p} \rightarrow t \\ p \rightarrow \infty}}\left[\left(I-h_{p} \Delta\right)^{-1}\left(I+h_{p} R\right)\right]^{k} u_{0}
$$

for $t \geqq 0$. Observe that $u\left(t ; u_{0}\right) \in X_{\varepsilon}^{+}$for $t \geqq 0$. Let $\tilde{u}_{0} \in X_{\varepsilon}^{+} \cap D(\Delta)$ and $\left\{\tilde{u}_{p, k}\right\}$ be the associate asymptotically approximate difference solution (defined as in (2.13)). Then we obtain the limit function $u\left(t ; \tilde{u}_{0}\right)$ in the same way as in (2.16). Moreover, noting that $\left\|u_{p, k}-\tilde{u}_{p, k}\right\| \leqq \|[(I-$ $\left.\left.h_{p} \Delta\right)^{-1}\left(I+h_{p} R\right)\right]^{k} u_{0}-\left[\left(I-h_{p} \Delta\right)^{-1}\left(I+h_{p} R\right)\right]^{k} \tilde{u}_{0}\left\|\leqq\left(1+h_{p} / 2 \sqrt{\varepsilon}\right)^{k}\right\| u_{0}-\tilde{u}_{0} \|$, we have

$$
\left\|u\left(t ; u_{0}\right)-u\left(t ; \tilde{u}_{0}\right)\right\| \leqq \exp (t / 2 \sqrt{\varepsilon})\left\|u_{0}-\tilde{u}_{0}\right\|, \quad t \geqq 0
$$

Set $T_{\varepsilon}(t) u_{0}=u\left(t ; u_{0}\right)$ for $t \geqq 0$ and $u_{0} \in X_{\varepsilon}^{+} \cap D(\Delta)$. Then, in view of (2.17) we can obtain a semigroup $\left\{T_{\varepsilon}(t) ; t \geqq 0\right\}$ of local type on $X_{\varepsilon}^{+}$. As is mentioned in the preceding example, $T_{\varepsilon}(t) u_{0}$ gives a unique weak solution of (DE) on every bounded subinterval of $[0, \infty)$.

On the other hand, we see in a way similar to Webb [25] that for each $u_{0} \in X_{\varepsilon}^{+}, T(t) u_{0}$ gives a unique mild solution of (2.12). In fact, $J_{p} \equiv\left(I-h_{p} \Delta\right)^{-1}\left(I+h_{p} R\right)=\left(I-h_{p} \Delta\right)^{-1}+h_{p}\left(I-h_{p} \Delta\right)^{-1} R$, and so,

$$
J_{p}^{k} u_{0}=\left(1-h_{p} \Delta\right)^{-k} u_{0}+h_{p} \sum_{i=0}^{k-1}\left(I-h_{p} \Delta\right)^{-(k-i)} R J_{p}^{i} u_{0}
$$

Hence,

$$
\begin{gathered}
\left\|J_{p}^{k} u_{0}-\left\{\mathrm{e}^{t \Delta} u_{0}+\sum_{i=0}^{k-1} h_{p} \mathrm{e}^{(k-1)} h_{p} \Delta R T_{\varepsilon}\left(i h_{p}\right) u_{0}\right\}\right\| \\
\leqq\left\|\left(I-h_{p} \Delta\right)^{-k} u_{0}-\mathrm{e}^{t \Delta} u_{0}\right\|+h_{p} \sum_{i=0}^{k-1}\left\|\left(I-h_{p} \Delta\right)^{-(k-i)}\left\{R J_{p}^{i} u_{0}-R T_{\varepsilon}\left(i h_{p}\right) u_{0}\right\}\right\| \\
+h_{p} \sum_{i=0}^{k-1}\left\|\left\{\left(I-h_{p} \Delta\right)^{-(k-i)}-\mathrm{e}^{(k-i) h_{p} \Delta}\right\} R T_{\varepsilon}\left(i h_{p}\right) u_{0}\right\|
\end{gathered}
$$

Set $k=\left[t / h_{p}\right]$. Since $\left(I-h_{p} \Delta\right)^{-\left[s / h_{p}\right]}$ converges to $\mathrm{e}^{s \Delta}$ as $p \rightarrow \infty$, uniformly on bounded subinterval of $[0, \infty)$ as well as on compact subsets of $X$, we have 


$$
u\left(t ; u_{0}\right)=\mathrm{e}^{t \Delta} u_{0}+\int_{0}^{t} \mathrm{e}^{(t-s) \Delta} R u\left(s ; u_{0}\right) \mathrm{ds} \quad \text { for } t \geqq 0
$$

by letting $p \rightarrow \infty$ in the above estimate and applying (2.16). The unicity of a strongly continuous solution of the integral equation (2.18) can be proved in a way similar to Webb [25; Proposition 3.6]. These facts mean that $T_{\varepsilon}(t) u_{0}$ is a unique mild solution.

Finally we note that

$$
\lim _{h \rightarrow+0} h^{-1}\left[T_{\varepsilon}(t) u-u\right]=A u \quad \text { for } u \in X_{\varepsilon}^{+} \cap D(\Delta) .
$$

Example 2.23. (Product Formula)

Let $A$ be the infinitesimal generator of a semigroup of linear contractions on a Banach space $X$ and let $B$ be a nonlinear dissipative operator in $X$ satisfying

(i) there exists a subset $D$ of $X$ with $D \subset D(A) \cap D(B)$,

(ii) $J_{h}=(I-h B)^{-1}(I-h A)^{-1}$ and $J_{h} D \subset D$ for $0<h<h_{0}$,

(iii) for every $u \in D$ and $\tau>0$, there exists a constant $M \equiv M(u, \tau)$ $>0$ such that

$$
\left\|A B J_{h}^{k} u\right\| \leqq M \quad \text { for } 0<h<h_{0} \text { and } 0 \leqq k h \leqq \tau .
$$

We want to consider the Cauchy problem for (DE) formulated to the operator $A+B$ of semilinear form:

$$
(\mathrm{d} / \mathrm{dt}) u(t)=(A+B) u(t), \quad t \geqq 0 ; u(0)=u_{0} .
$$

Webb [26] and Kobayashi [13] give some sufficient conditions guaranteeing the above conditions and some examples of the operators $A$ and $B$ satisfying (i), (ii) and (iii). For instance, let $X=L^{p}(0, \infty)$, where $1 \leqq p<\infty$, and $A u=-u^{\prime}, D(A)=\left\{u \in X ; u^{\prime} \in X\right\}$. Let $b(\cdot)$ be a continuously differentiable, nondecreasing function on $R^{1}$ such that $b(0)$ $=0$ and define an operator $B$ by $[B u](x)=b(u(x))$ for $u \in X$ such that $B u \in X$. Moreover, let $A_{1} u=-u^{\prime}$ for $u \in D\left(A_{1}\right)=\left\{u \in C[0, \infty) ; u^{\prime} \in C[0\right.$, $\infty)\}$, where $C[0, \infty)$ is the space of bounded uniformly continuous functions on $[0, \infty)$, and let $D=D(A) \cap D\left(A_{1}\right) \cap D(A B)$. Then, it is proved that (i), (ii) and (iii) hold for these $A, B$ and $D$.

Now, let $u_{0} \in D$ and $\tau>0$. We consider (DS) for (DE) on $[0, \tau]$ 
and show that the system $\left\{u_{p, k}\right\}$ defined by

$$
\begin{aligned}
& u_{p, k}=\left(I-h_{p} B\right)^{-1}\left(I-h_{p} A\right)^{-1} u_{p, k-1}, \quad k=1,2, \ldots, N_{p}=\left[\tau / h_{p}\right], \\
& u_{p, 0}=u_{0}, \quad h_{p} \downarrow 0,
\end{aligned}
$$

gives an asymptotically approximate difference solution of (DS) satisfying condition (S)'.

In fact, $(I-h A)(I-h B)=I-h(A+B)+h^{2} A B$ on $D(A B) \cap D(A)$, and so, $\left(I-h_{p}(A+B)+h_{p}^{2} A B\right) u_{p, k}=u_{p, k-1}$ for $1 \leqq k \leqq N_{p}$ and $p \geqq 1$. Hence,

$$
\begin{gathered}
\left\|u_{p, k}-h_{p}(A+B) u_{p, k}-u_{p, k-1}\right\|=\left\|-h_{p}^{2} A B u_{p, k}\right\| \leqq M h_{p}^{2} \text { for } 1 \leqq k \leqq N_{p} \\
\text { and } p \geqq 1
\end{gathered}
$$

Moreover, in view of Remark 2.9 we have

$$
\left\|u_{p, k}-u_{p, k-1}\right\| \leqq\left(2 M \tau+\left\|(A+B) u_{0}\right\|\right) h_{p} \text { for } 1 \leqq k \leqq N_{p} \text { and } p \geqq 1
$$

Consequently, we obtain the following result by employing Theorem 2.8:

Theorem 2.24. Under conditions (i), (ii) and (iii), we have:

(a) For each $u \in D$,

$$
T(t) u=\lim _{p \rightarrow \infty}\left[\left(I-h_{p} B\right)^{-1}\left(I-h_{p} A\right)^{-1}\right]^{\left[t / h_{p}\right]} u
$$

holds uniformly on bounded subintervals of $[0, \infty)$.

(b) Let $\bar{T}(t)$ be the continuous extension onto $\bar{D}$ of $T(t)$, for each $t>0$. Then $\{\bar{T}(t) ; t \geqq 0\}$ forms a semigroup of contractions on $\bar{D}$. Moreover, for each $u \in \bar{D}, \bar{T}(t) u$ gives a unique simple weak solution of (DE) on each bounded subinterval of $[0, \infty)$.

Remark. A converse of Theorem 2.24 holds: Assume that conditions (i) and (ii) hold. Let $u(t)$ be an $X$-valued function on $[0, \infty)$ such that $u(0)=u_{0}$. If for every $\tau>0, u(t)$ restricted to $[0, \tau]$ is a strong solution of $(2.20)$ on $[0, \tau]$ such that $\|A B u(t)\| \leqq M_{\tau}$ for almost every $t \in[0, \tau]$ and for some constant $M_{\tau}>0$, then $u(t)$ is represented as the product formula (2.22). See [13; Theorem 1.1]. 


\section{§3. Convergence of Difference Approximation}

In this section we prove Theorem 2.8 in several steps.

Throughout this section $A$ denotes a fixed operator in $X$ such that $A-\omega$ is dissipative for some $\omega \geqq 0$ and we suppose all of the assumptions of Theorem 2.8-I.

Let $v, v^{\prime}>0$ and let $h_{p}$ and $h_{q}$ be some fixed elements of the null sequence $\left\{h_{p}\right\}$ such that $h_{q} \leqq h_{p}$. Write $h=h_{p}$ and $h^{\prime}=h_{q}$ for simplicity, until before Theorem 3.9. Set

$$
\alpha=h^{\prime} / h, \beta=1-\alpha, \gamma=(1-\omega h)^{-1}, \alpha_{1}=\alpha \gamma \text { and } \beta_{1}=\beta \gamma \text {. }
$$

By $\left\{\left(u_{p, k}, v_{p, k}\right) ; k=0,1, \ldots, N_{p}, p=1,2, \ldots\right\} \quad$ and $\quad\left\{\left(u_{q, j}^{\prime}, v_{q, j}^{\prime}\right) ; j=0,1, \ldots\right.$, $\left.N_{q}, q=1,2, \ldots\right\}$, where $N_{p}=\left[(b-a) / h_{p}\right]$ and $N_{q}=\left[(b-a) / h_{q}\right]$, we denote the approximate difference solutions of (DS) with error bounds $v$ and $v^{\prime}$, respectively. Moreover, we write

$$
v_{p, k}=h_{p}\left\|\varepsilon_{p, k}^{v}\right\| \text { and } v_{q, j}^{\prime}=h_{q}\left\|\varepsilon_{q, j}^{v^{\prime}}\right\|,
$$

where $\varepsilon_{p, k}^{v}$ and $\varepsilon_{q, j}^{v^{\prime}}$ denote the errors at the $k$-th and $j$-th steps, respectively, and set

$$
a_{k, j}=\left\|u_{q, j}^{\prime}-u_{p, k}\right\| \quad \text { for } k, j \geqq 0 \text {. }
$$

First of all, condition (S) implies the following:

Lemma 3.1. Let $m \leqq n, a+m h \leqq b$ and let $a+n h^{\prime} \leqq b$. Then

$$
\begin{aligned}
& a_{m, 0} \leqq \sum_{k=1}^{m}\left\|u_{p, k}-u_{p, k-1}\right\| \leqq C \cdot\left(m h+\sum_{k=1}^{m} v_{p, k}\right), \\
& a_{0, n} \leqq \sum_{j=1}^{n}\left\|u_{q, j}^{\prime}-u_{q, j-1}^{\prime}\right\| \leqq C \cdot\left(n h^{\prime}+\sum_{j=1}^{n} v_{q, j}^{\prime}\right)
\end{aligned}
$$

Lemma 3.2. Let $1 \leqq k \leqq m$ and $1 \leqq j \leqq n$ for the same $m$ and $n$ as in Lemma 3.1. Then we have

$$
a_{k, j} \leqq \alpha_{1} a_{k-1, j-1}+\beta_{1} a_{k, j-1}+\gamma\left\{\alpha v_{p, k}+v_{q, j}^{\prime}\right\} .
$$

Proof. Applying Proposition 1.3 we have 


$$
\begin{aligned}
a_{k, j} \leqq & \alpha_{1}\left\|\left(u_{q, j}^{\prime}-h^{\prime} v_{q, j}^{\prime}\right)-\left(u_{p, k}-h v_{p, k}\right)\right\|+\beta_{1}\left\|\left(u_{q, j}^{\prime}-h^{\prime} v_{q, j}^{\prime}\right)-u_{p, k}\right\| \\
\leqq & \alpha_{1}\left\{\left\|\left(u_{q, j}^{\prime}-h^{\prime} v_{q, j}^{\prime}\right)-u_{q, j-1}^{\prime}\right\|+\left\|u_{q, j-1}^{\prime}-u_{p, k-1}\right\|+\| u_{p, k-1}\right. \\
& \left.-\left(u_{p, k}-h v_{p, k}\right) \|\right\}+\beta_{1}\left\{\left\|\left(u_{q, j}^{\prime}-h^{\prime} v_{q, j}^{\prime}\right)-u_{q, j-1}^{\prime}\right\|+\left\|u_{q, j-1}^{\prime}-u_{p, k}\right\|\right\} \\
\leqq & \alpha_{1} a_{k-1, j-1}+\beta_{1} a_{k, j-1}+\gamma\left\{\alpha v_{p, k}+v_{q, j}^{\prime}\right\} .
\end{aligned}
$$

Lemma 3.3. Let $a+n h^{\prime} \leqq b$. Then we have

$$
a_{1, n} \leqq \sum_{j=1}^{n} \alpha_{1} \beta_{1}^{j-1}\left(\begin{array}{c}
j-1 \\
1-1
\end{array}\right) a_{0, n-j}+\beta_{1}^{n}\left(\begin{array}{c}
n \\
0
\end{array}\right) a_{1,0}+\gamma^{n}\left\{v_{p, 1}+\sum_{j=0}^{n-1} v_{q, n-j}^{\prime}\right\}
$$

Proof. First, Lemma 3.2 yields that

$$
\begin{aligned}
a_{1, n} \leqq & \alpha_{1} a_{0, n-1}+\beta_{1}\left[\alpha_{1} a_{0, n-2}+\beta_{1} a_{1, n-2}+\gamma\left\{\alpha v_{p, 1}+v_{q, n-1}^{\prime}\right\}\right] \\
& +\gamma\left\{\alpha v_{p, 1}+v_{q, n}^{\prime}\right\} \\
\leqq & \alpha_{1} a_{0, n-1}+\alpha_{1} \beta_{1} a_{0, n-2}+\beta_{1}^{2} a_{1, n-2}+\gamma^{2} \cdot v_{p, 1} \alpha(1+\beta) \\
& +\gamma^{2} \cdot\left(v_{q, n}^{\prime}+v_{q, n-1}^{\prime}\right) .
\end{aligned}
$$

Suppose now that for $2 \leqq k<n$,

$$
\begin{aligned}
a_{1, n} \leqq & \sum_{j=1}^{k} \alpha_{1} \beta_{1}^{j-1}\left(\begin{array}{c}
j-1 \\
1-1
\end{array}\right) a_{0, n-j}+\beta_{1}^{k} a_{1, n-k}+\gamma^{k} \cdot v_{p, 1} \alpha\left(1+\beta+\cdots+\beta^{k-1}\right) \\
& +\gamma^{k} \cdot \sum_{j=0}^{k-1} v_{q, n-j}^{\prime} .
\end{aligned}
$$

Then, since

$$
\beta_{1}^{k} a_{1, n-k} \leqq \alpha_{1} \beta_{1}^{k}\left(\begin{array}{c}
k \\
1-1
\end{array}\right) a_{0, n-(k+1)}+\beta_{1}^{k+1} a_{1, n-(k+1)}+\gamma^{k+1} \cdot\left\{\alpha \beta^{k} v_{p, 1}+v_{q, n-k}^{\prime}\right\},
$$

we obtain

$$
\begin{aligned}
a_{1, n} \leqq & \sum_{j=1}^{k+1} \alpha_{1} \beta_{1}^{j-1}\left(\begin{array}{c}
j-1 \\
1-1
\end{array}\right) a_{0, n-j}+\beta_{1}^{k+1} a_{1, n-(k+1)}+\gamma^{k+1} \cdot v_{p, 1} \alpha \sum_{j=0}^{k} \beta^{j} \\
& +\gamma^{k+1} \cdot \sum_{j=0}^{k} v_{q, n-j}^{\prime} .
\end{aligned}
$$

Since $\alpha \sum_{j=0}^{k} \beta^{j} \leqq 1$, we have the assertion by induction. 
Difference Approximation

175

Lemma 3.4. Let $a+m h \leqq b$. Then,

$$
a_{m, m} \leqq \sum_{j=0}^{m} \alpha_{1}^{j} \beta_{1}^{m-j}\left(\begin{array}{c}
m \\
j
\end{array}\right) a_{m-j, 0}+\gamma^{m}\left\{\sum_{j=0}^{m-1} v_{p, m-j}+\sum_{j=0}^{m-1} v_{q, m-j}^{\prime}\right\}
$$

Proof. By Lemma 3.2,

$$
a_{m, m} \leqq \alpha_{1} a_{m-1, m-1}+\beta_{1} a_{m, m-1}+\gamma\left\{\alpha v_{p, m}+v_{q, m}^{\prime}\right\} .
$$

Suppose that for $2 \leqq k<m$,

$$
a_{m, m} \leqq \sum_{j=0}^{k}\left(\begin{array}{c}
k \\
j
\end{array}\right) \alpha_{1}^{j} \beta_{1}^{k-j} a_{m-j, m-k}+\gamma^{k}\left\{\sum_{j=0}^{k-1} v_{q, m-j}^{\prime}+\alpha \sum_{l=0}^{k-1} \sum_{j=0}^{l}\left(\begin{array}{l}
l \\
j
\end{array}\right) \alpha^{j} \beta^{l-j} v_{p, m-j}\right\} .
$$

Since

$$
a_{m-j, m-k} \leqq \alpha_{1} a_{m-j-1, m-k-1}+\beta_{1} a_{m-j, m-k-1}+\gamma\left\{\alpha v_{p, m-j}+v_{q, m-k}^{\prime}\right\},
$$

it follows that

$$
\begin{aligned}
a_{m, m} \leqq & \sum_{j=0}^{k}\left(\begin{array}{c}
k \\
j
\end{array}\right) \alpha_{1}^{j} \beta_{1}^{k-j}\left\{\alpha_{1} a_{m-j-1, m-k-1}+\beta_{1} a_{m-j, m-k-1}\right\} \\
& +\sum_{j=0}^{k}\left(\begin{array}{c}
k \\
j
\end{array}\right) \alpha_{1}^{j} \beta_{1}^{k-j} \gamma v_{q, m-k}^{\prime}+\gamma^{k} \cdot \sum_{j=0}^{k-1} v_{q, m-j}^{\prime} \\
& +\gamma \cdot \alpha \sum_{j=0}^{k}\left(\begin{array}{c}
k \\
j
\end{array}\right) \alpha_{1}^{j} \beta_{1}^{k-j} v_{p, m-j}+\gamma^{k} \cdot \alpha \sum_{l=0}^{k-1} \sum_{j=0}^{l}\left(\begin{array}{l}
l \\
j
\end{array}\right) \alpha^{j} \beta^{l-j} v_{p, m-j} \\
\leqq & \sum_{j=0}^{k+1}\left(\begin{array}{c}
k+1 \\
j
\end{array}\right) \alpha_{1}^{j} \beta_{1}^{k+1-j} a_{m-j, m-(k+1)} \\
& +\gamma^{k+1}\left\{\sum_{j=0}^{k} v_{q, m-j}^{\prime}+\alpha \sum_{l=0}^{h} \sum_{j=0}^{l}\left(\begin{array}{l}
l \\
j
\end{array}\right) \alpha^{j} \beta^{l-j} v_{p, m-j}\right\} .
\end{aligned}
$$

Therefore, by induction

$$
a_{m, m} \leqq \sum_{j=0}^{m} \alpha^{j} \beta^{m-j}\left(\begin{array}{c}
m \\
j
\end{array}\right) a_{m-j, 0}+\gamma^{m}\left\{\sum_{j=0}^{m-1} v_{q, m-j}^{\prime}+\alpha \sum_{l=0}^{m-1} \sum_{j=0}^{l}\left(\begin{array}{l}
l \\
j
\end{array}\right) \alpha^{j} \beta^{l-j} v_{p, m-j}\right\} .
$$

Now, we estimate the last term of the right side. First we observe that

$$
\alpha \sum_{l=0}^{m-1} \sum_{j=0}^{l}\left(\begin{array}{l}
l \\
j
\end{array}\right) \alpha^{j} \beta^{l-j} v_{p, m-j} \leqq \sum_{j=0}^{m-1} v_{p, m-j} \alpha^{j+1} \sum_{l=j}^{\infty}\left(\begin{array}{l}
l \\
j
\end{array}\right) \beta^{l-j} .
$$


If $\beta=1$, then $\alpha=0$ and hence we have the desired estimate. We then assume that $0<\beta<1$. Then,

$$
(1-\beta)^{-j-1}=\sum_{n=0}^{\infty}\left(\begin{array}{c}
-j-1 \\
n
\end{array}\right)(-\beta)^{n}=\sum_{n=0}^{\infty}\left(\begin{array}{c}
n+j \\
n
\end{array}\right) \beta^{n}
$$

Setting $n=l-j$, we obtain $\left(\begin{array}{c}n+j \\ n\end{array}\right)=\left(\begin{array}{l}l \\ j\end{array}\right)$ : hence $\sum_{l=j}^{\infty}\left(\begin{array}{l}l \\ j\end{array}\right) \beta^{l-j}=(1-\beta)^{-j-1}$ $=\alpha^{-j-1}$. This completes the proof.

Q.E.D.

Lemma 3.5. Let $1 \leqq m \leqq n, a+m h \leqq b$ and let $a+n h^{\prime} \leqq b$. Then

$$
\begin{aligned}
a_{m, n} \leqq & \sum_{j=0}^{m}\left(\begin{array}{c}
n \\
j
\end{array}\right) \alpha_{1}^{j} \beta_{1}^{n-j} a_{m-j, 0}+\sum_{j=m}^{n}\left(\begin{array}{c}
j-1 \\
m-1
\end{array}\right) \alpha_{1}^{m} \beta_{1}^{j-m} a_{0, n-j} \\
& +\gamma^{n}\left\{\sum_{j=0}^{m-1} v_{p, m-j}+\sum_{j=0}^{n-1} v_{q, n-j}^{\prime}\right\} .
\end{aligned}
$$

Proof. In view of Lemmas 3.2, 3.3 and 3.4, it is sufficient to show that $a_{m+1, n+1}$ satisfies the inequality with $m$ and $n$ replaced by $m+1$ and $n+1$ respectively, under the assumption that $a_{m, n}$ and $a_{m+1, n}$ satisfy the corresponding inequalities. By Lemma 3.2, we have

$$
\begin{aligned}
& a_{m+1, n+1} \leqq \alpha_{1} a_{m, n}+\beta_{1} a_{m+1, n}+\gamma\left\{\alpha v_{p, m+1}+v_{q, n+1}^{\prime}\right\} \\
& \leqq \\
& \quad \sum_{j=0}^{m} \alpha_{1}^{j+1} \beta_{1}^{n-j}\left(\begin{array}{c}
n \\
j
\end{array}\right) a_{m-j, 0}+\sum_{j=0}^{m+1} \alpha_{1}^{j} \beta_{1}^{n+1-j}\left(\begin{array}{c}
n \\
j
\end{array}\right) a_{m+1-j, 0} \\
& \quad+\sum_{j=m}^{n} \alpha_{1}^{m+1} \beta_{1}^{j-m}\left(\begin{array}{c}
j-1 \\
m-1
\end{array}\right) a_{0, n-j}+\sum_{j=m+1}^{n} \alpha_{1}^{m+1} \beta_{1}^{j-(m+1)+1}\left(\begin{array}{c}
j-1 \\
m
\end{array}\right) a_{0, n-J} \\
& \quad+\alpha_{1} \gamma \cdot \sum_{j=0}^{n-1} v_{q, n-j}^{\prime}+\beta_{1} \gamma^{n} \cdot \sum_{j=0}^{n-1} v_{q, n-j}^{\prime}+\gamma v_{q, n+1}^{\prime} \\
& \quad+\alpha_{1} \gamma^{n} \cdot \sum_{j=0}^{m-1} v_{p, m-j}+\beta_{1} \gamma^{n} \cdot \sum_{j=0}^{m} v_{p, m+1-j}+\gamma \alpha v_{p, m+1} \\
& \leqq \\
& \quad \sum_{j=0}^{m+1} \alpha_{1}^{j} \beta_{1}^{n+1-j}\left(\begin{array}{c}
n+1 \\
j
\end{array}\right) a_{m+1-j, 0}+\sum_{j=m+1}^{n+1} \alpha_{1}^{m+1} \beta_{1}^{j-(m+1)}\left(\begin{array}{c}
j-1 \\
(m+1)-1
\end{array}\right) a_{0, n+1-j} \\
& \quad+\gamma^{n+1} \cdot \sum_{j=0}^{(n+1)-1} v_{q, n+1-j}^{\prime}+\gamma^{n+1}\left\{\alpha \sum_{j=1}^{m} v_{p, m+1-j}+\beta \sum_{j=1}^{m} v_{p, m+1-j}\right. \\
& \left.\quad+\beta v_{p, m+1}+\alpha v_{p, m+1}\right\} .
\end{aligned}
$$


This means that $a_{m+1, n+1}$ satisfies the inequality in question with $m$ and $n$ replaced by $m+1$ and $n+1$ respectively.

Q.E.D.

Lemma 3.6. Let $1 \leqq m \leqq n, a+m h \leqq b$ and let $a+n h^{\prime} \leqq b$. Then we have
(a) $\sum_{j=0}^{m}\left(\begin{array}{c}n \\ j\end{array}\right) \alpha^{j} \beta^{n-j} \leqq 1$,
(b) $\sum_{j=m}^{n}\left(\begin{array}{c}j-1 \\ m-1\end{array}\right) \alpha^{m} \beta^{j-m} \leqq 1$
(c) $\sum_{j=0}^{m}\left(\begin{array}{c}n \\ j\end{array}\right) \alpha^{j} \beta^{n-j}(m-j) \leqq\left\{(n \alpha-m)^{2}+n \alpha \beta\right\}^{1 / 2}$,
(d) $\sum_{j=m}^{n}\left(\begin{array}{r}j-1 \\ m-1\end{array}\right) x^{m} \beta^{j-m}(n-j) \leqq\left\{m \beta / \alpha^{2}+(m \beta / \alpha+m-n)^{2}\right\}^{1 / 2}$.

Proof. (a) and (b) are easily seen. The proofs of (c) and (d) are stated in Crandall-Liggett [6; Lemma 1.4].

Combining this lemma with the estimate given in Lemma 3.5, we have the following:

Lemma 3.7. Let $1 \leqq m \leqq n, a+m h \leqq b$ and let $a+n h^{\prime} \leqq b$. Then,

$$
\begin{aligned}
\left\|u_{q, n}^{\prime}-u_{p, m}\right\| & \leqq \exp \left(2 \omega n h^{\prime}\right)\left[C\left\{\left(n h^{\prime}-m h\right)^{2}+n h^{\prime}\left(h-h^{\prime}\right)\right\}^{1 / 2}\right. \\
& \left.+C\left\{m h\left(h-h^{\prime}\right)+\left(m h-n h^{\prime}\right)^{2}\right\}^{1 / 2}+(1+C)\left(v+v^{\prime}\right)\right] .
\end{aligned}
$$

Proof. In view of Lemmas 3.5 and 3.1, we have

$$
\begin{aligned}
a_{m, n} \leqq & \sum_{j=0}^{m}\left(\begin{array}{c}
n \\
j
\end{array}\right) \alpha_{1}^{j} \beta_{1}^{n-j} C\left\{(m-j) h+\sum_{k=1}^{m-j} v_{p, k}\right\}+\sum_{j=m}^{n}\left(\begin{array}{r}
j-1 \\
m-1
\end{array}\right) \alpha_{1}^{m} \beta_{1}^{j-m} C\left\{(n-j) h^{\prime}\right. \\
& \left.+\sum_{k=1}^{n-j} v_{, q, k}\right\}+\gamma^{n} C\left\{\sum_{j=0}^{m-1} v_{p, m-j}+\sum_{j=0}^{n-1} v^{\prime}{ }_{q, n-j}\right\} \\
\leqq & \gamma^{n} \sum_{j=0}^{m}\left(\begin{array}{c}
n \\
j
\end{array}\right) \alpha^{j} \beta^{n-j}(m-j) C+\sum_{j=m}^{n} \gamma^{j}\left(\begin{array}{c}
j-1 \\
m-1
\end{array}\right) \alpha^{m} \beta^{j-m}(n-j) C_{2} h^{\prime} \\
& +C v \gamma^{n} \sum_{j=0}^{m}\left(\begin{array}{c}
n \\
j
\end{array}\right) \alpha^{j} \beta^{n-j}+C v^{\prime} \sum_{j=m}^{n} \gamma^{j}\left(\begin{array}{c}
j-1 \\
m-1
\end{array}\right) \alpha^{m} \beta^{j-m}+\gamma^{n}\left\{v+v^{\prime}\right\}
\end{aligned}
$$


Since $\gamma^{n} \leqq \exp \left(2 \omega n h^{\prime}\right)$, we have the assertion by applying Lemma 3.6 to this estimate.

Q.E.D.

Remark 3.8. It should be noted that the right side of the estimate given in Lemma 3.7 is independent of the choice of approximate solutions $\left\{u_{p, k}\right\}$ and $\left\{u_{q, j}^{\prime}\right\}$ of (DS).

Now, we are in position to prove the first half of Theorem 2.8.

Theorem 3.9. Let $A$ be an operator in $X$ such that $A-\omega$ is dissipative for some $\omega \geqq 0$. Let $J$ be a bounded interval whose end points are $a$ and $b$ and let $u_{0} \in \overline{D(A)}$. Assume that for every $v>0$, the approximating difference scheme (DS) has an approximate difference solution $\left\{\left(u_{p, k}, v_{p, k}\right)\right\}$ with error bound $v$. If condition (S) is satisfied, then we have:

(a) For every null sequence $v_{i} \downarrow 0$ there exists a subsequence $\left\{p_{i}\right\}$ and

$$
u(t)=\lim _{\substack{a+k h_{p_{i}} \rightarrow t \\ i \rightarrow \infty}} u_{p_{i}, k}^{v_{i}}
$$

exists for $t \in \bar{J}=[a, b]$.

(b) Given a $v>0$, let $\left\{\left(\tilde{u}_{p, k}, \tilde{v}_{p, k}\right)\right\} \subset G(A)$ be an approximate difference solution of (DS) with error bound $v$ satisfying (S), then for every sequence $\left\{m_{p} \tilde{h}_{p}\right\}$ converging to $t \in \bar{J}$,

$$
\varlimsup_{a+m_{p} \tilde{h}_{p} \rightarrow t}\left\|u(t)-\tilde{u}_{p, m_{p}}\right\| \leqq \exp (2 \omega(b-a))(1+C) v .
$$

(c) $u(t)$ is Lipschitz continuous on $\bar{J}$, that is, $\|u(t)-u(s)\| \leqq$ $C|t-s|$ for $t, s \in \bar{J}$.

Proof. (a) Let $v_{i} \downarrow 0$. For each $v_{i}$, let $\left\{\left(u_{p, k}^{i}, v_{p, k}^{i}\right)\right\}_{p \geqq p_{i}}$ be an approximate difference solution with error bound $v_{i}$, where $p_{i}$ is the integer associated with $v_{i}$ through condition $\left(\alpha_{1}\right)$. Let $a, b(a<b)$ be the end points of $J$ and let

$$
u_{i}(t)=u_{p_{i}, k_{i}(t)}^{i}, k_{i}(t)=\left[(t-a) / h_{p_{i}}\right] \text { for } t \in J
$$

Then $k_{i}(t) h_{p_{i}} \uparrow t-a$ as $i \rightarrow \infty$ and Lemma 3.7 yields that 


$$
\begin{aligned}
& \left\|u_{i}(t)-u_{j}(t)\right\| \cdot \exp (2 \omega(a-t)) \\
& \leqq \\
& \quad C\left(\left\{k_{i}(t) h_{p_{i}}-k_{j}(t) h_{p_{j}}\right\}^{2}+h_{p_{i}} k_{i}(t)\left(h_{p_{j}}-h_{p_{i}}\right)\right)^{1 / 2} \\
& \quad+C\left(h_{p_{j}} k_{j}(t)\left(h_{p_{j}}-h_{p_{i}}\right)+\left\{k_{j}(t) h_{p_{J}}-k_{i}(t) h_{p_{i}}\right\}^{2}\right)^{1 / 2} \\
& \quad+(1+C)\left(v_{i}+v_{j}\right) \text { for } i \geqq j .
\end{aligned}
$$

Hence, $\lim _{i, j \rightarrow \infty}\left\|u_{i}(t)-u_{j}(t)\right\|=0$ uniformly for $t \in \bar{J}$. We set

$$
u(t)=\lim _{i \rightarrow \infty} u_{i}(t), \quad t \in \bar{J} .
$$

(b) Let $\left\{\left(\tilde{u}_{p, k}, \tilde{v}_{p, k}\right)\right\}$ be any approximate difference solution of (DS) with error bound $v$ satisfying conditions $\left(\alpha_{2}\right)$ and (S) for the same constant $C$ as in (a). Then by Lemma 3.7,

$$
\begin{aligned}
& \exp (2 \omega(a-t))\left\|u_{i}(t)-\tilde{u}_{p, m}\right\| \\
& \leqq \\
& \quad C\left(\left\{k_{i}(t) h_{p_{i}}-m h_{p}\right\}^{2}+(t-a)\left(h_{p}-h_{p_{i}}\right)\right)^{1 / 2} \\
& \quad+C\left(m h_{p}\left(h_{p}-h_{p_{i}}\right)+\left\{m h_{p}-k_{i}(t) h_{p_{i}}\right\}^{2}\right)^{1 / 2} \\
& \quad+(1+C)\left(v_{i}+v\right)
\end{aligned}
$$

for $i$ sufficiently large. Letting $i \rightarrow \infty$, we obtain

$$
\begin{aligned}
& \exp (2 \omega(a-b))\left\|u(t)-\tilde{u}_{p, m}\right\| \leqq 2 C\left\{\left(t-a-m h_{p}\right)^{2}+(b-a) h_{p}\right\}^{1 / 2} \\
& \quad+(1+C) v .
\end{aligned}
$$

From this it follows that $\varlimsup_{a+m_{p} h_{p} \rightarrow t}\left\|u(t)-\tilde{u}_{p, m_{p}}\right\| \leqq \exp (2 \omega(b-a))(1+C) v$ for every sequence $\left\{m_{p} h_{p}\right\}$ converging to $t \in \bar{J}$.

(c) Let $s, t \in \bar{J}, s<t$. Then, condition (S) yields that

$$
\begin{aligned}
\left\|u_{i}(t)-u_{i}(s)\right\| & \leqq \sum_{k=k_{i}(s)}^{k_{i}(t)-1}\left\|u_{p_{i}, k+1}^{i}-u_{p_{i}, k}^{i}\right\| \\
& \leqq C \sum_{k_{i}(s)}^{k_{i}(t)-1}\left(1+\left\|\varepsilon_{p_{i}, k}^{v_{i}}\right\|\right) h_{p_{i}} \\
& \leqq C\left(k_{i}(t)-k_{i}(s)\right) h_{p_{i}}+C \nu_{i} .
\end{aligned}
$$

$\begin{array}{ll}\text { Letting } i \rightarrow \infty \text {, we have that }\|u(t)-u(s)\| \leqq C|t-s| \text {. } & \text { Q.E.D. }\end{array}$ 
Theorem 3.10. Let $A$ be an operator in $X$ such that $A-\omega$ is dissipative for some $\omega \geqq 0$. Let $J$ be a bounded interval whose end points are $a$ and $b$ and let $u_{0} \in \overline{D(A)}$. Assume that (DS) admits an asymptotically approximate difference solution $\left\{\left(u_{p, k}, v_{p, k}\right)\right\}$ satisfying condition (S)'. Then we have:

(a)

$$
u(t)=\lim _{\substack{a+m h_{p} \rightarrow t \\ p \rightarrow \infty}} u_{p, m}
$$

holds for every $t \in \bar{J}$ and $u(t)$ does not depend on the choice of the approximate solutions $\left\{\left(u_{p, k}, v_{p, k}\right)\right\}$ of (DS).

(b) $\|u(t)-u(s)\| \leqq C|t-s|$ for $t, s \in \bar{J}$, where $C$ is the constant given by condition $(\mathrm{S})^{\prime}$.

Proof. (a) Let $h_{q} \leqq h_{p}$ and $1 \leqq m \leqq n$. Then by Lemmas 3.5 and 3.6,

$$
\begin{aligned}
& \left\|u_{q, n}-u_{p, m}\right\| \leqq \sum_{j=0}^{m}\left(\begin{array}{c}
n \\
j
\end{array}\right) \alpha_{1}^{j} \beta_{1}^{n-j} C\left\{(m-j) h_{p}+(b-a) \max _{1 \leqq k \leqq m}\left\|\varepsilon_{p, k}\right\|\right\} \\
& \quad+\sum_{j=m}^{n}\left(\begin{array}{c}
j-1 \\
m-1
\end{array}\right) \alpha_{1}^{m} \beta_{1}^{j-m} C\left\{(n-j) h_{q}+(b-a) \max _{1 \leqq j \leqq n}\left\|\varepsilon_{q, j}\right\|\right\} \\
& \quad+\gamma \cdot(b-a)\left\{\max _{1 \leqq k \leqq m}\left\|\varepsilon_{p, k}\right\|+\max _{1 \leqq j \leqq n}\left\|\varepsilon_{q, j}\right\|\right\} \\
& \leqq \\
& \quad \gamma^{n} \sum_{j=0}^{m}\left(\begin{array}{c}
n \\
j
\end{array}\right) \alpha^{j} \beta^{n-j}(m-j) C h_{p}+\sum_{j=m}^{n} \gamma^{j}\left(\begin{array}{c}
j-1 \\
m-1
\end{array}\right) \alpha^{m} \beta^{j-m}(n-j) C h_{q} \\
& \quad+C(b-a) \max _{1 \leqq k \leqq m}\left\|\varepsilon_{p, k}\right\| \gamma^{n} \sum_{j=0}^{m}\left(\begin{array}{c}
n \\
j
\end{array}\right) \alpha^{j} \beta^{n-j} \\
& \quad+C(b-a) \max _{1 \leqq j \leqq n}\left\|\varepsilon_{q, j}\right\| \sum_{j=m}^{n} \gamma^{j}\left(\begin{array}{c}
j-1 \\
m-1
\end{array}\right) \alpha^{m} \beta^{j-m} \\
& \quad+\gamma^{n} \cdot(b-a)\left\{\max _{1 \leqq k \leqq m}\left\|\varepsilon_{p, k}\right\|+\max _{1 \leqq j \leqq n}\left\|\varepsilon_{q, j}\right\|\right\} .
\end{aligned}
$$

Now, we set $u_{p}(t)=u_{p, k_{p}(t)}, k_{p}(t)=\left[(t-a) / h_{p}\right]$ for $t \in \bar{J}$. Then, $k_{p}(t) h_{p}$ $\uparrow t-a$ and

$$
\begin{aligned}
& \left\|u_{p}(t)-u_{q}(t)\right\| \exp (2 \omega(a-t)) \\
& \quad \leqq C\left(\left\{k_{p}(t) h_{p}-k_{p}(t) h_{q}\right\}^{2}+h_{p} k_{p}(t)\left(h_{p}-h_{q}\right)\right)^{1 / 2}
\end{aligned}
$$




$$
\begin{aligned}
& +C\left(h_{q} k_{q}(t)\left(h_{p}-h_{q}\right)+\left\{k_{q}(t) h_{q}-k_{p}(t) h_{p}\right\}^{2}\right)^{1 / 2} \\
& +(b-a)(1+C)\left\{\max _{1 \leqq k \leqq N_{p}}\left\|\varepsilon_{p, k}\right\|+\max _{1 \leqq j \leqq N_{q}}\left\|\varepsilon_{q, i}\right\|\right\} .
\end{aligned}
$$

Hence, $\lim _{p, q \rightarrow \infty}\left\|u_{p}(t)-u_{q}(t)\right\|=0$ holds uniformly for $t \in \bar{J}$. Set

$$
u(t)=\lim _{p \rightarrow \infty} u_{p}(t), \quad t \in \bar{J}
$$

Then we have

$$
\begin{aligned}
&\left\|u(t)-u_{p, m}\right\| \exp (2 \omega(a-t)) \\
& \leqq C\left\{\left(t-a-m h_{p}\right)^{2}+(t-a) h_{p}\right\}^{1 / 2}+C\left\{m h_{p}^{2}+\left(m h_{p}-t+a\right)^{2}\right\}^{1 / 2} \\
&+(b-a)(1+C) \max _{1 \leqq k \leqq m}\left\|\varepsilon_{p, k}\right\| .
\end{aligned}
$$

Thus, (a) is proved. (b) follows from Theorem 3.9 (c). Q.E.D.

\section{§4. Abstract Cauchy Problem}

In this section we investigate some properties of weak solutions of (DE) and discuss the uniqueness of a solution of WCP. Moreover, we consider the relationship between the generalized domain $\hat{D}(A)$ and the Lipschitz continuity of weak solutions of (DE).

Throughout this section we assume that $A$ is an operator in a Banach space $X$ such that $A-\omega$ is dissipative for some $\omega \geqq 0$.

We start with the following:

Lemma 4.1. Let $u(t)$ be a simple weak solution of (DE) on a closed interval $J=[a, b]$. Then for every pair $s, t \in J$ with $s \leqq t$ and every $(x, y) \in G(A)$, the following inequality holds:

$$
\mathrm{e}^{-2 \omega t}\|u(t)-x\|^{2}-\mathrm{e}^{-2 \omega s}\|u(s)-x\|^{2} \leqq 2 \int_{s}^{t} \mathrm{e}^{-2 \omega \tau}<y, u(\tau)-x>_{s} \mathrm{~d} \tau
$$

Moreover, (4.1) holds for $s, t \in J$ with $s \leqq t$ and $(x, y) \in G(\hat{A})$ by letting $<,>_{s}$ be the functional on $X^{* *} \times X^{* *}$. 
Proof. Let $\left\{h_{p}\right\},\left\{u_{p}(t)\right\}$ and $\left\{v_{p}(t)\right\}$ be the corresponding null sequence and sequences of $X$-valued, strongly measurable functions satisfying conditions $(\mathrm{i})_{s}-(\text { iii })_{s}$. Put

$$
\psi_{p}(t)=h_{p}^{-1}\left\{u_{p}(t)-u_{p}\left(t-h_{p}\right)\right\}-v_{p}(t) .
$$

First, let $(x, y) \in G(\hat{A})$. Define an operator $A_{1}$ in $X^{* *}$ by $G\left(A_{1}\right)=$ $G(A) \cup\{(x, y)\}$. Take an $\varepsilon>0$ sufficiently small. Since $A_{1}-\omega$ is dissipative,

$$
<v_{p}(\tau)-y, u_{p}(\tau)-x>_{i} \leqq \omega\left\|u_{p}(\tau)-x\right\|^{2} \quad \text { for a.a. } \tau \in J .
$$

Since $v_{p}(\tau)-y=h_{p}^{-1}\left\{u_{p}(\tau)-x\right\}-h_{p}^{-1}\left\{u_{p}\left(\tau-h_{p}\right)-x\right\}-y-\psi_{p}(\tau)$ for a.a. $\tau \in$ $J_{\varepsilon}=[a+\varepsilon, b]$ and $h_{p}<\varepsilon,(4.3)$ and Proposition 1.1 imply the following estimate:

$$
\begin{aligned}
& \omega\left\|u_{p}(\tau)-x\right\|^{2} \\
& \geqq h_{p}^{-1}\left\|u_{p}(\tau)-x\right\|^{2}-<h_{p}^{-1}\left\{u_{p}\left(\tau-h_{p}\right)-x\right\}+y+\psi_{p}(\tau), u_{p}(\tau)-x>_{s} \\
& \geqq h_{p}^{-1}\left\|u_{p}(\tau)-x\right\|^{2}-h_{p}^{-1}\left\|u_{p}\left(\tau-h_{p}\right)-x\right\| \cdot\left\|u_{p}(\tau)-x\right\| \\
& \quad \quad-<y, u_{p}(\tau)-x>_{s}-\left\|\psi_{p}(\tau)\right\| \cdot\left\|u_{p}(\tau)-x\right\| \\
& \geqq\left(2 h_{p}\right)^{-1}\left\|u_{p}(\tau)-x\right\|^{2}-\left(2 h_{p}\right)^{-1}\left\|u_{p}\left(\tau-h_{p}\right)-x\right\|^{2}-<y, u_{p}(\tau)-x>_{s} \\
& \quad-\left\|\psi_{p}(\tau)\right\| \cdot\left\|u_{p}(\tau)-x\right\|
\end{aligned}
$$

for almost all $\tau \in J_{\varepsilon}$. On the other hand, $\left\|u_{p}(\tau)-x\right\|$ is uniformly essentially bounded on $J$, and so, a constant $M>0$ can be found such that

$$
\left\|u_{p}(\tau)-x\right\| \leqq M \quad \text { for almost all } \tau \in J \text { and all } n \text {. }
$$

Let $s, t \in J_{\varepsilon}, s \leqq t$, and set

$$
t_{k}^{p}=a+k h_{p}, \quad k=0,1, \ldots, N_{p}=\left[(b-a) / h_{p}\right] ; h_{p}<\varepsilon .
$$

Let $m$ and $n$ be such that $s \in\left[t_{m-1}^{p}, t_{m}^{p}\right]$ and $t \in\left[t_{n}^{p}, t_{n+1}^{p}\right]$. Then by (4.4),

$$
\begin{aligned}
2 \omega \int_{t_{m}^{p}}^{t_{n}^{p}} \mathrm{e}^{-2 \omega \tau}\left\|u_{p}(\tau)-x\right\|^{2} \mathrm{~d} \tau+2 \int_{t_{m}^{p}}^{t_{n}^{p}} \mathrm{e}^{-2 \omega \tau}<y, u_{p}(\tau)-x>_{s} \mathrm{~d} \tau \\
+2 M \int_{t_{m}^{p}}^{t_{n}^{p}}\left\|\psi_{p}(\tau)\right\| \mathrm{d} \tau
\end{aligned}
$$




$$
\begin{aligned}
\geqq & h_{p}^{-1} \int_{t_{n-1}^{p}}^{t_{n}^{p}} \mathrm{e}^{-2 \omega \tau}\left\|u_{p}(\tau)-x\right\|^{2} \mathrm{~d} \tau-h_{p}^{-1} \int_{t_{m-1}^{p}}^{t_{m}^{p}} \mathrm{e}^{-2 \omega\left(\tau+h_{p}\right)}\left\|u_{p}(\tau)-x\right\|^{2} \mathrm{~d} \tau \\
& +h_{p}^{-1} \int_{t_{m}^{p}}^{t_{n-1}^{p}}\left(\mathrm{e}^{-2 \omega \tau}-\mathrm{e}^{-2 \omega\left(\tau+h_{p}\right)}\right)\left\|u_{p}(\tau)-x\right\|^{2} \mathrm{~d} \tau
\end{aligned}
$$

Since $\int_{t_{m}^{p}}^{t_{n}^{p}}\left\|\psi_{p}(\tau)\right\| d \tau \leqq \int_{J_{\varepsilon}}\left\|\psi_{p}(\tau)\right\| d \tau \rightarrow 0$ and $u_{p}(\cdot) \rightarrow u(\cdot)$ in $L^{\infty}\left(J_{\varepsilon} ; X\right)$ (and hence, in $\left.L^{\infty}\left(J_{\varepsilon} ; X^{* *}\right)\right)$ as $p \rightarrow \infty$, and since $<,>_{s}: X^{* *} \times X^{* *} \rightarrow R^{1}$ is upper-semicontinuous, the limit superior of (4.6) is not greater than

$$
2 \omega \int_{s}^{t} \mathrm{e}^{-2 \omega \tau}\|u(\tau)-x\|^{2} d \tau+2 \int_{s}^{t} \mathrm{e}^{-2 \omega \tau}<y, u(\tau)-x>_{s} \mathrm{~d} \tau
$$

On the other hand, $h_{p}^{-1}\left(\mathrm{e}^{-2 \omega \tau}-\mathrm{e}^{-2 \omega\left(\tau+h_{p}\right)}\right) \rightarrow 2 \omega \mathrm{e}^{-2 \omega \tau}$ as $p \rightarrow \infty$, uniformly on $J_{\varepsilon}$, and so, the right side of (4.6) tends to

$$
\mathrm{e}^{-2 \omega t}\|u(t)-x\|^{2}-\mathrm{e}^{-2 \omega s}\|u(s)-x\|^{2}+2 \omega \int_{s}^{t} \mathrm{e}^{-2 \omega \tau}\|u(\tau)-x\|^{2} \mathrm{~d} \tau
$$

From this we obtain the inequality (4.1) by letting $p \rightarrow \infty$ in (4.6). Since $\varepsilon>0$ was arbitrary and $u(t)$ is strongly continuous on $[a, b]$, we have the required inequality (4.1) for every pair $s, t \in J$ with $s \leqq t$. Next, the proof of (4.1) in the case of $(x, y) \in G(A)$ is similarly proved; note that in this case we can take $A$ itself, instead of $A_{1}$. E.D.

Remark. Let $u(t)$ be as in Lemma 4.1. Then we can prove the following inequality in a way similar to the above: For every $s, t \in J$ with $s \leqq t$ and $(x, y) \in G(A)$,

$$
\begin{aligned}
\|u(t)-x\|^{2}-\|u(s)-x\|^{2} \leqq & 2 \omega \int_{s}^{t}\|u(\tau)-x\|^{2} \mathrm{~d} \tau \\
& +2 \int_{s}^{t}<y, u(\tau)-x>_{s} \mathrm{~d} \tau .
\end{aligned}
$$

Note that (4.7) holds for $s, t \in J$ with $s \leqq t$ and $(x, y) \in G(\hat{A})$ by letting $<,>_{s}$ be the functional on $X^{* *} \times X^{* *}$.

Lemma 4.2. Let $u(t)$ and $\tilde{u}(t)$ be simple weak solutions of (DE) on intervals $J=[a, b]$ and $\tilde{J}=[\tilde{a}, \tilde{b}]$, respectively. Then 


$$
\begin{aligned}
& \int_{\alpha}^{\beta}\left\{\|\tilde{u}(t)-u(\sigma)\|^{2}-\|\tilde{u}(s)-u(\sigma)\|^{2}\right\} \mathrm{d} \sigma \\
& \quad+\int_{s}^{t}\left\{\|\tilde{u}(\tau)-u(\beta)\|^{2}+\|\tilde{u}(\tau)-u(\alpha)\|^{2}\right\} \mathrm{d} \tau \\
& \leqq 2 \omega \int_{s}^{t} \mathrm{~d} \tau \int_{\alpha}^{\beta}\|\tilde{u}(\tau)-u(\sigma)\|^{2} \mathrm{~d} \sigma
\end{aligned}
$$

holds for every $\alpha, \beta \in J$ with $\alpha \leqq \beta$ and every $s, t \in \tilde{J}$ with $s \leqq t$.

Proof. Let $\left\{h_{p}\right\},\left\{u_{p}(t)\right\}$ and $\left\{v_{p}(t)\right\}$ be the corresponding null sequence and sequences of $X$-valued, strongly measurable functions on $J$ satisfying conditions (i) $-(\text { iii) })_{s}$. Let $\left\{\psi_{p}(t)\right\}$ be the functions defined by (4.2). Since $\left(u_{p}(\sigma), \quad v_{p}(\sigma)\right) \in G(A) \quad(\subset G(\hat{A}))$ for almost all $\sigma \in J$, (4.7) in the Remark after Lemma 4.1 implies that

$$
\begin{aligned}
\| \tilde{u}(t)- & u_{p}(\sigma)\left\|^{2}-\right\| \tilde{u}(s)-u_{p}(\sigma) \|^{2} \\
& \leqq 2 \int_{s}^{t}<v_{p}(\sigma), \tilde{u}(\tau)-u_{p}(\sigma)>_{s} \mathrm{~d} \tau+2 \omega \int_{s}^{t}\left\|\tilde{u}(\tau)-u_{p}(\sigma)\right\|^{2} \mathrm{~d} \tau
\end{aligned}
$$

for $s, t \in \tilde{J}$ with $s \leqq t$ and for almost all $\sigma \in J$. Let $0<\varepsilon<b-a$ and $h_{p}<$ $\varepsilon$. Then, it follows from Proposition 1.1 that

$$
\begin{aligned}
&<v_{p}(\sigma), \tilde{u}(\tau)-u_{p}(\sigma)>_{s} \\
& \leqq<h_{p}^{-1}\left\{\tilde{u}(\tau)-u_{p}\left(\sigma-h_{p}\right)\right\}-h_{p}^{-1}\left\{\tilde{u}(\tau)-u_{p}(\sigma)\right\}, \tilde{u}(\tau)-u_{p}(\sigma)>_{s} \\
&+<-\psi_{p}(\sigma), \tilde{u}(\tau)-u_{p}(\sigma)>_{s} \\
& \leqq-\left(2 h_{p}\right)^{-1}\left\|\tilde{u}(\tau)-u_{p}(\sigma)\right\|^{2}+\left(2 h_{p}\right)^{-1}\left\|\tilde{u}(\tau)-u_{p}\left(\sigma-h_{p}\right)\right\|^{2} \\
&+\left\|-\psi_{p}(\sigma)\right\| \cdot\left\|\tilde{u}(\tau)-u_{p}(\sigma)\right\|
\end{aligned}
$$

for a.a. $\sigma \in J_{\varepsilon}=[a+\varepsilon, b]$ and a.a. $\tau \in \tilde{J}$. Let $\alpha, \beta \in J_{\varepsilon}, \alpha \leqq \beta$. Let $t_{k}^{p}$ be the points of $J$ defined by (4.5) and let $m$ and $n$ be such that $\alpha \in\left[t_{m-1}^{p}, t_{m}^{p}\right]$ and $\beta \in\left[t_{n}^{p}, t_{n+1}^{p}\right]$. Then, applying (4.10) produces

$$
\begin{aligned}
2 \int_{t_{m}^{p}}^{t_{n}^{p}} & <v_{p}(\sigma), \tilde{u}(\tau)-u(\sigma)>_{s} \mathrm{~d} \sigma \\
& \leqq-h_{p}^{-1} \int_{t_{n-1}^{p}}^{t_{n}^{p}}\left\|\tilde{u}(\tau)-u_{p}(\sigma)\right\|^{2} \mathrm{~d} \sigma+h_{p}^{-1} \int_{t_{m-1}^{p}}^{t_{m}^{p}}\left\|\tilde{u}(\tau)-u_{p}(\sigma)\right\|^{2} \mathrm{~d} \sigma
\end{aligned}
$$




$$
+2 \int_{t_{m}^{p}}^{t_{n}^{p}}\left\|\psi_{p}(\sigma)\right\| \cdot\left\|\tilde{u}(\tau)-u_{p}(\sigma)\right\| \mathrm{d} \sigma
$$

Combining this with (4.9), we obtain

$$
\begin{aligned}
& \int_{t_{m}^{p}}^{t_{n}^{p}}\left\{\left\|\tilde{u}(t)-u_{p}(\sigma)\right\|^{2}-\left\|\tilde{u}(s)-u_{p}(\sigma)\right\|^{2}\right\} \mathrm{d} \sigma \\
\leqq & -\int_{s}^{t} \mathrm{~d} \tau \int_{t_{n-1}^{p}}^{t_{n}^{p}} h_{p}^{-1}\left\|\tilde{u}(\tau)-u_{p}(\sigma)\right\|^{2} \mathrm{~d} \sigma+\int_{s}^{t} \mathrm{~d} \tau \int_{t_{m-1}^{p}}^{t_{m}^{p}} h_{p}^{-1}\left\|\tilde{u}(\tau)-u_{p}(\sigma)\right\|^{2} \mathrm{~d} \sigma \\
& +2 \omega \int_{s}^{t} \mathrm{~d} \tau \int_{t_{m}^{p}}^{t_{n}^{p}}\left\|\tilde{u}(\tau)-u_{p}(\sigma)\right\|^{2} \mathrm{~d} \sigma+2 \int_{s}^{t} \mathrm{~d} \tau \int_{t_{m}^{p}}^{t_{n}^{p}}\left\|\psi_{p}(\sigma)\right\| \cdot\left\|\tilde{u}(\tau)-u_{p}(\sigma)\right\| \mathrm{d} \sigma .
\end{aligned}
$$

Therefore, letting $p \rightarrow \infty$, we have the inequality (4.8) for $\alpha, \beta \in J_{\varepsilon}$ with $\alpha \leqq \beta$. Since $\varepsilon>0$ was arbitrary and $u(t)$ is strongly continuous on $J$, we have the assertion.

Q.E.D.

Lemma 4.3. Let $u(t)$ and $\hat{u}(t)$ be two weak solutions of (DE) on bounded intervals $J$ and $\tilde{J}$, respectively. Then, the inequality (4.8) holds for every $\alpha, \beta \in J$ with $\alpha \leqq \beta$ and every $s, t \in \tilde{J}$ with $s \leqq t$.

Proof. Let $\quad\left\{J_{k}^{p} ; J_{k}^{p}=\left[a_{k}^{p}, b_{k}^{p}\right], k=1,2, \ldots, N_{p}, p=1,2, \ldots\right\}, \quad\left\{\tilde{J}_{k}^{q} ; \tilde{J}_{k}^{q}=\right.$ $\left.\left[\tilde{a}_{k}^{q}, \tilde{b}_{k}^{q}\right], k=1,2, \ldots, N_{q}, q=1,2, \ldots\right\}, \quad\left\{u_{p}(t)\right\}$ and $\left\{\tilde{u}_{q}(t)\right\}$ be the corresponding families of closed subsets of $J$ and $\tilde{J}$ and sequences of $X$ valued strongly measurable functions on $J$ and $\tilde{J}$ satisfying (i)-(iv) in condition (II), respectively. Let $\alpha, \beta \in J, \alpha \leqq \beta$ and for each $p$, choose $\alpha^{p}$ in some $\left[a_{m}^{p}, b_{m}^{p}\right]$ and $\beta^{p}$ in some $\left[a_{n}^{p}, b_{n}^{p}\right]$ so that $\alpha^{p}-\alpha=\inf \{t-\alpha$; $\left.t \in J^{p}=\cup J_{k}^{p}, t \geqq \alpha\right\}$ and $\beta-\beta^{p}=\inf \left\{\beta-t ; t \in J^{p}, t \leqq \beta\right\}$. First, we fix a $q$ and assume that both $s$ and $t$ belong to an interval $\tilde{J}_{l}^{q}=\left[\tilde{a}_{l}^{q}, \tilde{b}_{l}^{q}\right]$. Then by Lemma 4.2,

$$
\begin{aligned}
& \int_{a_{k}^{p}}^{b_{k}^{p}}\left\{\left\|\tilde{u}_{q}(t)-u_{p}(\sigma)\right\|^{2}-\left\|\tilde{u}_{q}(s)-u_{p}(\sigma)\right\|^{2}\right\} \mathrm{d} \sigma \\
& \quad+\int_{s}^{t}\left\{\left\|\tilde{u}_{q}(\tau)-u_{p}\left(b_{k}^{p}\right)\right\|^{2}-\left\|\tilde{u}_{q}(\tau)-u_{p}\left(a_{k}^{p}\right)\right\|^{2}\right\} \mathrm{d} \tau \\
& \leqq 2 \omega \int_{s}^{t} \mathrm{~d} \tau \int_{a_{k}^{p}}^{b_{k}^{p}}\left\|\tilde{u}_{q}(\tau)-u_{p}(\sigma)\right\|^{2} \mathrm{~d} \sigma, \quad k=m, m+1, \ldots, n,
\end{aligned}
$$


where we set $a_{m}^{p}=\alpha^{p}$ and $b_{n}^{p}=\beta^{p}$. Therefore, we have

$$
\begin{aligned}
& \sum_{k=m}^{n} \int_{a_{k}^{p}}^{b_{k}^{p}}\left\{\left\|\tilde{u}_{q}(t)-u_{p}(\sigma)\right\|^{2}-\left\|\tilde{u}_{q}(s)-u_{p}(\sigma)\right\|^{2}\right\} \mathrm{d} \sigma \\
& \quad+\int_{s}^{t}\left\{\left\|\tilde{u}_{q}(\tau)-u_{p}\left(\beta^{p}\right)\right\|^{2}-\left\|\tilde{u}_{q}(\tau)-u_{p}\left(\alpha^{p}\right)\right\|^{2}\right\} \mathrm{d} \tau \\
& \leqq \\
& 2 \omega \int_{s}^{t} \mathrm{~d} \tau \sum_{k=m}^{n} \int_{a_{k}^{p}}^{b_{k}^{p}}\left\|\tilde{u}(\tau)-u_{p}(\sigma)\right\|^{2} \mathrm{~d} \sigma \\
& \quad-\sum_{k=m}^{n-1} \int_{s}^{t}\left\{\left\|\tilde{u}_{q}(\tau)-u_{p}\left(b_{k}^{p}\right)\right\|^{2}-\left\|\tilde{u}_{q}(\tau)-u_{p}\left(a_{k+1}^{p}\right)\right\|^{2}\right\} \mathrm{d} \tau
\end{aligned}
$$

Since $\left\|u_{p}(\sigma)\right\|$ and $\left\|\tilde{u}_{q}(\tau)\right\|$ are uniformly bounded with respect to $(\sigma, \tau) \in$ $J^{p} \times \tilde{J}^{q}$ and $p, q=1,2, \ldots$, the second term on the right side of (4.13) is majorized by

$$
M|t-s| \sum_{k=m}^{n-1}\left\|u_{p}\left(b_{k}^{p}\right)-u_{p}\left(a_{k+1}^{p}\right)\right\|
$$

for some constant $M>0$. Since $u(\sigma)$ is strongly continuous on the closed interval $[\alpha, \beta]$, a constant $M^{\prime}>0$ can be found such that

$$
\begin{aligned}
& \left|\sum_{k=m}^{n} \int_{a_{k}^{p}}^{b_{k}^{p}}\left\|\tilde{u}_{q}(\tau)-u_{p}(\sigma)\right\|^{2} \mathrm{~d} \sigma-\int_{\alpha}^{\beta}\left\|\tilde{u}_{q}(\tau)-u(\sigma)\right\|^{2} \mathrm{~d} \sigma\right| \\
& \leqq M^{\prime}\left\{\sup _{\sigma \in J^{p}}\left\|u_{p}(\sigma)-u(\sigma)\right\|+\sum_{k=0}^{N_{p}}\left(a_{k+1}^{p}-b_{k}^{p}\right)+\left(\alpha-\alpha^{p}\right)+\left(\beta-\beta^{p}\right)\right\} \\
& \equiv M_{p}^{\prime}, \text { for all } \tau \in[s, t] .
\end{aligned}
$$

Similarly, the difference between the first term on the left side of (4.13) and

$$
\int_{\alpha}^{\beta}\left\{\left\|\tilde{u}_{q}(t)-u(\sigma)\right\|^{2}-\left\|\tilde{u}_{q}(s)-u(\sigma)\right\|^{2}\right\} \mathrm{d} \sigma
$$

is majorized by $2 M_{p}^{\prime}$.

Note that $\alpha^{p} \rightarrow \alpha$ and $\beta^{p} \rightarrow \beta$ as $p \rightarrow \infty$. Hence, it follows from the definition that $u_{p}\left(\alpha^{p}\right) \rightarrow u(\alpha)$ and $u_{p}\left(\beta^{p}\right) \rightarrow u(\beta)$ as $p \rightarrow \infty$. Consequently, letting $p \rightarrow \infty$ in (4.13) we see that 


$$
\begin{aligned}
& \int_{\alpha}^{\beta}\left\{\left\|\tilde{u}_{q}(t)-u(\sigma)\right\|^{2}-\left\|\tilde{u}_{q}(s)-u(\sigma)\right\|^{2}\right\} \mathrm{d} \sigma \\
& \quad+\int_{s}^{t}\left\{\left\|\tilde{u}_{q}(\tau)-u(\beta)\right\|^{2}-\left\|\tilde{u}_{q}(\tau)-u(\alpha)\right\|^{2}\right\} \mathrm{d} \tau \\
& \leqq 2 \omega \int_{s}^{t} \mathrm{~d} \tau \int_{\alpha}^{\beta}\left\|\tilde{u}_{q}(\tau)-u(\sigma)\right\|^{2} \mathrm{~d} \sigma .
\end{aligned}
$$

This inequality always holds if the $s$ and $t$ belong to the same interval $\tilde{J}_{k}^{q}$. Therefore, we can prove by applying this inequality, instead of (4.12), and by a routine argument that the inequality (4.8) holds for every pair $s, t \in \tilde{J}$ with $s \leqq t$.

Q.E.D.

Now, applying a method due to Bénilan [2; Lemma 1.2], we obtain the following result:

Theorem 4.4. Let $u(t)$ and $\tilde{u}(t)$ be weak solutions of (DE) on a bounded interval $J$. Then

$$
e^{-\omega t}\|u(t)-\tilde{u}(t)\| \leqq e^{-\omega s}\|u(s)-\tilde{u}(s)\| \quad \text { for } s, t \in J \text { with } s \leqq t .
$$

Proof. Let $a$ and $b$ be the left and right end points of $J$, respectively. Set

$$
\phi(\tau, \sigma)= \begin{cases}\|\hat{u}(\tau)-u(\sigma)\|^{2} & \text { if }(\tau, \sigma) \in J \times J, \\ 0 & \text { otherwise }\end{cases}
$$

and then consider the following regularization of $\phi$ :

$$
\phi_{\varepsilon}(\tau, \sigma)=\left(\rho_{\varepsilon} * \phi\right)(\tau, \sigma)=\iint_{R^{2}} \rho_{\varepsilon}(\tau-\bar{\tau}, \sigma-\bar{\sigma}) \phi(\bar{\tau}, \bar{\sigma}) \mathrm{d} \bar{\tau} \mathrm{d} \bar{\sigma}
$$

where $\rho_{\varepsilon}(\xi, \eta)=\varepsilon^{2} \rho(\varepsilon \xi) \rho(\varepsilon \eta), \quad(\xi, \eta) \in R^{2}$, and $\rho$ is a modifier such that $\rho \in C_{0}^{\infty}\left(R^{1}\right), \quad \rho \geqq 0, \quad \operatorname{supp}[\rho] \subset[-1,1], \quad \int_{R^{1}} \rho \mathrm{d} \xi=1$ and $\rho$ is symmetric. Let $0<\varepsilon_{0}<(b-a) / 2$. Let $a+\varepsilon_{0} \leqq s \leqq t \leqq b-\varepsilon_{0}$ and $a+\varepsilon_{0} \leqq \alpha \leqq \beta \leqq b-\varepsilon_{0}$. Then applying Lemma 4.3, we have

$$
\int_{\alpha}^{\beta}\left\{\phi_{\varepsilon}(t, \sigma)-\phi_{\varepsilon}(s, \sigma)\right\} \mathrm{d} \sigma+\int_{s}^{t}\left\{\phi_{\varepsilon}(\tau, \beta)-\phi_{\varepsilon}(\tau, \alpha)\right\} \mathrm{d} \tau
$$




$$
\leqq 2 \omega \int_{s}^{t} \int_{\alpha}^{\beta} \phi_{\varepsilon}(\tau, \sigma) \mathrm{d} \sigma \mathrm{d} \tau
$$

Thus, in a way similar to Bénilan [2; Lemma 1.2], we have

$$
\phi_{\varepsilon}(t, t)-\phi_{\varepsilon}(s, s) \leqq 2 \omega \int_{s}^{t} \phi_{\varepsilon}(\tau, \tau) d \tau
$$

This implies that $\mathrm{e}^{-2 \omega t} \phi_{\varepsilon}(t, t) \leqq \mathrm{e}^{-2 \omega s} \phi_{\varepsilon}(s, s)$. Therefore, letting $\varepsilon \downarrow 0$, we have $\mathrm{e}^{-2 \omega t} \phi(t, t) \leqq \mathrm{e}^{-2 \omega s} \phi(s, s)$ for $a+\varepsilon_{0} \leqq s \leqq t \leqq b-\varepsilon_{0}$. Since $\varepsilon_{0}$ was arbitrary and since $u(t)$ and $\tilde{u}(t)$ are strongly continuous on $J$, we have the assertion.

Q.E.D.

Remarks. (1) Theorem 4.4 guarantees the uniqueness of a solution of WCP. Hence Theorem 2.3 is valid.

(2) Theorem 4.4 states that the $u(t)$ mentioned in a remark after the statement of condition (II) gives a unique weak solution of (DE) on $[a, c)$.

The proof of Theorem 2.8 is given by Theorems 3.9, 3.10 and the following:

Corollary 4.5. Each of the limit function $u(t)$ obtained by Theorems 3.9 and 3.10 gives a unique simple weak solution of (DE) associated with the initial-value $u_{0}$.

Proof. Let $u(t)$ be the limit function obtained by Theorem 3.9 (a). Let $u_{i}(t)$ be the functions defined by (3.1) and let

$$
v_{i}(t)=v_{p_{\imath}, h_{\imath}(t), h_{\imath}(t)}^{i}=\left[(t-a) / h_{p_{i}}\right], \quad a+h_{p_{i}} \leqq t \leqq b, \quad i=1,2, \ldots
$$

Then, $u_{i}(t)$ and $v_{i}(t)$ are step functions and $v_{i}(t) \in A u_{i}(t)$ for all $t$. Theorem 3.9 (a) states that $u(t)=\lim _{i \rightarrow \infty} u_{i}(t)$ holds uniformly on $J$, so $u_{i}(\cdot) \rightarrow u(\cdot)$ in $L^{\infty}(J ; X)$ as $i \rightarrow \infty$. Since

$$
h_{p_{i}}^{-1}\left\{u_{i}(t)-u_{i}\left(t-h_{p_{\imath}}\right)\right\}-v_{i}(t)=\varepsilon_{p_{\imath}, k_{\imath}(t)}^{i}, a+h_{p_{i}} \leqq t \leqq b,
$$

we have

$$
\int_{t_{k}^{i}}^{t_{k+1}^{i}}\left\|h_{p_{\imath}}^{-1}\left\{u_{i}(t)-u_{i}\left(t-h_{p_{\imath}}\right)\right\}-v_{i}(t)\right\| \mathrm{d} t=h_{p_{\imath}}\left\|\varepsilon_{p_{\imath}, k}^{i}\right\| \quad 1 \leqq k \leqq N_{p_{\imath}}-1
$$


where $t_{k}^{i}=a+k h_{p_{i}}$. Therefore, for every $\varepsilon>0$,

$$
\int_{a+\varepsilon}^{b}\left\|h_{p_{i}}^{-1}\left\{u_{i}(t)-u_{i}\left(t-h_{p_{\imath}}\right)\right\}-v_{i}(t)\right\| \mathrm{d} t \leqq v_{i} \longrightarrow 0 \quad \text { as } \quad i \longrightarrow \infty
$$

From this and Theorem 3.9 (a) it follows that $u(t)$ is a unique weak solution of (DE) associated with the initial-value $u_{0}$ Q.E.D.

Since $u(t)$ gives a unique weak solution, the limit $u(t)$ does not depend on the choice of the sequence $\left\{v_{i}\right\}$, and hence, Theorem 2.8 is valid.

Now, we investigate some properties of weak solutions in the sequel. First we show that a weak solution is an integral solution in the sense of Definition 2.2.

Theorem 4.6. Let $u(t)$ be a weak solution of (DE) on a bounded interval $J$. Then for every pair $s, t \in J$ with $s \leqq t$ and every $(x, y) \in$ $G(A)$, the inequality (4.1) holds:

$$
\mathrm{e}^{-2 \omega t}\|u(t)-x\|^{2}-\mathrm{e}^{-2 \omega s}\|u(s)-x\|^{2} \leqq 2 \int_{s}^{t} \mathrm{e}^{-2 \omega \sigma}<y, u(\sigma)-x>_{s} \mathrm{~d} \sigma .
$$

Moreover, (4.1) holds for $s, t \in J$ with $s \leqq t$ and $(x, y) \in G(\hat{A})$ by letting $<,>_{s}$ be the functional from $X^{* *} \times X^{* *}$ to $R^{1}$.

Proof. Let $\left\{J_{k}^{p} ; J_{k}^{p}=\left[a_{k}^{p}, b_{k}^{p}\right], k=1,2, \ldots, N_{p}, p=1,2, \ldots\right\}$ and $\left\{u_{p}(t)\right\}$ be the corresponding family of closed subsets of $J$ and sequence of $X$ valued, strongly measurable functions on $J$ satisfying (i)-(iv) in condition (II), respectively. Let $s, t \in J, s \leqq t$ and for each $p$, choose $s^{p}$ and $t^{p}$ in $J^{p}=\cup_{k} J_{k}^{p}$ so that $s^{p}-s=\inf \left\{\sigma-s ; \sigma \geqq s, \sigma \in J^{p}\right\}$ and $t-t^{p}=\inf \{t-\sigma$; $\left.\sigma \leqq t, \sigma \in J^{p}\right\}$. Let $s^{p} \in\left[a_{m}^{p}, b_{m}^{p}\right]$ and $t^{p} \in\left[a_{n}^{p}, b_{n}^{p}\right]$. First, let $(x, y) \in G(\hat{A})$. Then making use of Lemma 4.1, we have

$$
\begin{aligned}
& \mathrm{e}^{-2 \omega t^{p}}\left\|u_{p}\left(t^{p}\right)-x\right\|^{2}-\mathrm{e}^{-2 \omega s^{p}}\left\|u_{p}\left(s^{p}\right)-x\right\|^{2} \\
& \leqq 2 \sum_{k=m}^{n} \int_{\alpha_{k}^{p}}^{\beta_{k}^{p}} \mathrm{e}^{-2 \omega \sigma}<y, u_{p}(\sigma)-x>_{s} \mathrm{~d} \sigma \\
& \quad-\sum_{k=m}^{n-1}\left\{\mathrm{e}^{\left.-2 \omega \beta_{k}^{p}\left\|u_{p}\left(\beta_{k}^{p}\right)-x\right\|^{2}-\mathrm{e}^{-2 \omega \alpha_{k+1}^{p}}\left\|u_{p}\left(\alpha_{k+1}^{p}\right)-x\right\|^{2}\right\},}\right.
\end{aligned}
$$


where $\alpha_{m}^{p}=s^{p}, \quad \beta_{n}^{p}=t^{p}, \quad \alpha_{k}^{p}=a_{k}^{p} \quad$ for $k=m+1, \quad m+2, \ldots, n$, and $\beta_{k}^{p}=b_{k}^{p}$ for $k=m, m+1, \ldots, n-1$. Moreover,

$$
\begin{aligned}
& \sum_{k=m}^{n-1}\left|\mathrm{e}^{-2 \omega b_{k}^{p}}\left\|u_{p}\left(b_{k}^{p}\right)-x\right\|^{2}-\mathrm{e}^{-2 \omega a_{k+1}^{p}}\left\|u_{p}\left(a_{k+1}^{p}\right)-x\right\|^{2}\right| \\
& \leqq \sum_{k=1}^{N_{p}-1}\left|\left\|u_{p}\left(b_{k}^{p}\right)-x\right\|^{2}-\left\|u_{p}\left(a_{k+1}^{p}\right)-x\right\|^{2}\right| \\
& +\sum_{k=1}^{N_{p}-1}\left(1-\mathrm{e}^{-2 \omega\left(a_{k+1}^{p}-b_{k}^{p}\right)}\right)\left\|u_{p}\left(a_{k+1}^{p}\right)-x\right\|^{2} \\
& \leqq \text { const. }\left(\sum_{k=1}^{N_{p}-1}\left\|u_{p}\left(b_{k}^{p}\right)-u_{p}\left(a_{k+1}^{p}\right)\right\|+\sum_{k=1}^{N_{p}-1}\left(a_{k+1}^{p}-b_{k}^{p}\right)\right) .
\end{aligned}
$$

The right side goes to 0 as $p \rightarrow \infty$ by (iii) in condition (II). Since $s^{p} \rightarrow s$ and $t^{p} \rightarrow t$ as $p \rightarrow \infty$, we have the required inequality by letting $p \rightarrow \infty$ in (4.15). Next, the proof of (4.1) in the case of $(x, y) \in G(A)$ is quite similar.

Q.E.D.

Remarks. (1) We have shown in the above theorem that a weak solution of (DE) satisfies the inequality (4.1). We can also prove by a way similar to the proofs mentioned above that a weak solution $u(t)$ of (DE) on $J$ satisfies the inequality (4.7):

$$
\|u(t)-x\|^{2}-\|u(s)-x\|^{2} \leqq 2 \int_{s}^{t}<y, u(\sigma)-x>_{s} d \sigma+2 \omega \int_{s}^{t}\|u(\sigma)-x\|^{2} \mathrm{~d} \sigma
$$

for $s, t \in J$ with $s \leqq t$ and $(x, y) \in G(A)$, and at the same time that for every $s, t \in J$ with $s \leqq t$ and every $(x, y) \in G(\hat{A})$ by letting $<,>_{s}$ be the functional on $X^{* *} \times X^{* *}$.

(2) Let $u(t)$ be a strongly absolutely continuous function on $J$. Then, $u(t)$ satisfies (4.1) if and only if it satisfies (4.7). In fact, suppose (4.1) holds. Then we have for $t \in J$ and $h>0$ sufficiently small

$$
\begin{gathered}
h^{-1}\left(\mathrm{e}^{-2 \omega(t+h)}-\mathrm{e}^{-2 \omega t}\right)\|u(t+h)-x\|^{2} \\
\quad+\mathrm{e}^{-2 \omega t} h^{-1}\left\{\|u(t+h)-x\|^{2}-\|u(t)-x\|^{2}\right\} \\
\leqq h^{-1} \int_{t}^{t+h} \mathrm{e}^{-2 \omega \sigma}<y, u(\sigma)-x>{ }_{s} \mathrm{~d} \sigma .
\end{gathered}
$$


Since $\|u(t)-x\|$ is absolutely continuous on $J$, we have

$$
\frac{\mathrm{d}}{\mathrm{d} t}\|u(t)-x\|^{2} \leqq<y, u(t)-x>_{s}+2 \omega\|u(t)-x\|^{2}, \quad \text { a.a. } t \in J,
$$

by letting $h \downarrow 0$, from which (4.7) follows. The converse is similarly proved. As is seen from this fact, we can employ (4.7) to give a definition of integral solution, instead of (4.1).

Theorem 4.7. Let $\left\{u^{(l)}(t)\right\}$ be a sequence of weak solutions of (DE) on a bounded interval $J$. Suppose that $u^{(l)}(t)$ converges to $u(t)$ as $l \rightarrow \infty$, uniformly on $J$. Then the limit $u(t)$ on $J$ gives a weak solution of (DE) on $J$.

Proof. For each $u^{(l)}(t)$, let $\left\{u_{p}^{(l)}(t)\right\}$ and $\left\{J_{k}^{p}(l)\right\}$ be the corresponding sequence of functions on $J$ and family of closed subintervals $J_{k}^{p}(l)$ satisfying conditions (i)-(iv). Let $J_{k}^{p}(l)=\left[a_{k}^{p}(l), b_{k}^{p}(l)\right], k=1,2, \ldots, N_{p}(l), p, l=$ $1,2, \ldots$. Then there exist subsequences $\left\{l_{i}\right\}$ and $\left\{p_{i}\right\}$ such that

$$
\begin{aligned}
& \sup _{t \in J}\left\|u^{\left(l_{i}\right)}(t)-u(t)\right\| \leqq 1 / i \\
& \sup _{t \in J^{p_{i}\left(l_{i}\right)}}\left\|u_{p_{i}}^{\left(l_{i}\right)}(t)-u^{\left(l_{i}\right)}(t)\right\| \leqq 1 / i, \quad J^{p_{i}}\left(l_{i}\right)=\bigcup_{k} J_{k}^{p_{i}}\left(l_{i}\right) \\
& \sum_{k=0}^{N_{p_{i}\left(l_{i}\right)}}\left(a_{k+1}^{p_{i}}\left(l_{i}\right)-b_{k}^{p_{i}}\left(l_{i}\right)\right) \leqq 1 / i \text { and } \sum_{k=1}^{N_{p_{i}}\left(l_{i}\right)-1}\left\|u_{p_{i}}\left(a_{k+1}^{p_{i}}\left(l_{i}\right)\right)-u_{p_{i}}\left(b_{k}^{p_{i}}\left(l_{i}\right)\right)\right\| \\
& \leqq 1 / i
\end{aligned}
$$

Clearly, $u(t)$ is strongly continuous on $J$ and $u_{p_{i}}^{\left(l_{i}\right)} \mid J_{k}^{p_{i}}\left(l_{i}\right)$ is a simple weak solution of (DE) on $J_{k}^{p_{i}}\left(l_{\imath}\right)$ for each $k=1,2, \ldots, N_{p_{i}}\left(l_{i}\right)$ and $i=$ $1,2,3, \ldots$ Hence, $u(t)$ gives a weak solution of (DE) on $J$. Q.E.D.

Corollary 4.8. Suppose that $A-\omega$ is dissipative for some $\omega \geqq 0$. Let $S \subset \overline{D(A)}$ and $J=[a, b)$. If for every $v \in S$, (DE) on $J$ has a weak solution $u(t ; v)$ with the initial-value $v$, then for every $w \in \bar{S}$, there exists a unique weak solution $u(t ; w)$ of (DE) on $J$ such that $u(a ; w)=$ $w$. 
Proof. Let $w \in \bar{S}$ and let $\left\{w_{p}\right\}$ be a sequence in $S$ such that $w_{p} \rightarrow w$. Then, Theorem 4.4 yields that $\left\|u\left(t ; w_{q}\right)-u\left(t ; w_{p}\right)\right\| \leqq \mathrm{e}^{\omega(b-a)}\left\|w_{q}-w_{p}\right\| \rightarrow 0$ as $p, q \rightarrow \infty$. Hence, $u(t ; w)=\lim _{p \rightarrow \infty} u\left(t ; w_{p}\right)$ exists uniformly for $t \in J$. Therefore, it follows from Theorem 4.7 that $u(t ; w)$ becomes a unique weak solution of (DE) on $J$.

Q.E.D.

Next, we give some necessary and sufficient conditions for a weak solution to be Lipschitz continuous.

Theorem 4.9. Let $J=[a, b)$ and $u(t)$ be an $X$-valued function on $J$ satisfying (4.7) and $u(t) \in \overline{D(A)}$ for all $t \in J$, and let $u$ sonsider the following conditions:

(L.1) $u(t)$ is Lipschitz continuous on $J$;

(L.2) $u(t) \in D(\hat{A})$ for all $t \in J$ and $\lim _{t \uparrow b} u(t) \in \hat{D}(A)$;

(L.3) $u(a) \in D(\widehat{A})$;

(L.4) $u(a) \in \hat{D}(A)$.

Then (L.1) $\Rightarrow(\mathrm{L} .2) \Rightarrow(\mathrm{L} .3) \Rightarrow(\mathrm{L} .4)$. If $u(t)$ is a weak solution of (DE) on $J$, then the above conditions together with the following condition are equivalent:

(L.5) $u(t) \in D(\hat{A})$ for all $t \in J$ and

$\lim _{h \downarrow 0} h^{-1}\|u(t+h)-u(t)\|=\|\widehat{A} u(t)\| \leqq \mathrm{e}^{\omega(t-a)}\|\widehat{A} u(a)\| \quad$ for all $t \in J$.

Moreover, in this case, we have

$$
\|u(t)-u(s)\| \leqq \mathrm{e}^{\omega(b-a)}\|\hat{A} u(a)\||\cdot| t-s \mid \quad \text { for } t, s \in J .
$$

Proof. First, let $u(t)$ be an $X$-valued function on $J$ satisfying (4.7) and $u(t) \in \overline{D(A)}$ for all $t \in J$. Assume that (L.1) holds, i.e., there exists a constant $M>0$ with

$$
\|u(t)-u(s)\| \leqq M|t-s| \quad \text { for } \quad t, s \in J
$$

Fix any $t_{0} \in J$ and set

$$
\alpha\left(t_{0}\right)=\lim _{h \downarrow 0} h^{-1}\left\|u\left(t_{0}+h\right)-u\left(t_{0}\right)\right\| .
$$


Then by assumption, $\alpha\left(t_{0}\right) \leqq M$. Let $z\left(t_{0}\right) \in X^{* *}$ be any weak-star limit point of the set $\left\{h^{-1}\left(u\left(t_{0}+h\right)-u\left(t_{0}\right)\right)\right\}$. Then $\left\|z\left(t_{0}\right)\right\| \leqq \alpha\left(t_{0}\right)$. By the way, (4.7) and Proposition 1.1 yield that for every $(x, y) \in G(A)$,

$$
\begin{aligned}
& <h^{-1}\left(u\left(t_{0}+h\right)-u\left(t_{0}\right)\right), w> \\
& \leqq(2 h)^{-1}\left\{\left\|u\left(t_{0}+h\right)-x\right\|^{2}-\left\|u\left(t_{0}\right)-x\right\|^{2}\right\} \\
& \leqq h^{-1} \int_{t_{0}}^{t_{0}+h}<y, u(\tau)-x>_{s} \mathrm{~d} \tau+\omega h^{-1} \int_{t_{0}}^{t_{0}+h}\|u(\tau)-x\|^{2} \mathrm{~d} \tau
\end{aligned}
$$

for $w \in F\left(u\left(t_{0}\right)-x\right)$ and $h>0$. Therefore, we have

$$
<z\left(t_{0}\right)-y, u\left(t_{0}\right)-x>_{i} \leqq \omega\left\|u\left(t_{0}\right)-x\right\|^{2} .
$$

This states that $\left(u\left(t_{0}\right), z\left(t_{0}\right)\right) \in G(\hat{A})$, and so,

$$
M \geqq \alpha\left(t_{0}\right) \geqq\left\|\hat{A} u\left(t_{0}\right)\right\|
$$

Since $u(t)$ is Lipschitz continuous on $J, \lim _{t \uparrow b} u(t)=u(b)$ exists. Since $t_{0}$ was arbitrary in $J$, it follows fiom (4.17) that $u(b) \in \hat{D}(A)$, and so, (L.2) follows. It is clear that (L.2) $\Rightarrow($ L.3 $\Rightarrow$ (L.4). Next, suppose $u(t)$ be a weak solution of (DE) on $J$ satisfying (4.16). Then, we have shown that condition (L.2) holds. Let $t_{0} \in J$ and take a $z \in \hat{A} u\left(t_{0}\right)$. Let $\left(u\left(t_{0}\right), z\left(t_{0}\right)\right)=(x, y)$ in (4.1). Then Theorem 4.6 implies

$$
\mathrm{e}^{-2 \omega t}\left\|u(t)-u\left(t_{0}\right)\right\|^{2}-\mathrm{e}^{-2 \omega s}\left\|u(s)-u\left(t_{0}\right)\right\|^{2} \leqq 2 \int_{s}^{t} \mathrm{e}^{-2 \omega \sigma}<z, u(\sigma)-u\left(t_{0}\right)>_{s} \mathrm{~d} \sigma
$$

for $s, t \in J$ with $s \leqq t$. From this it follows (Brezis [3; Lemma 51]) that

$$
\mathrm{e}^{-\omega t}\left\|u(t)-u\left(t_{0}\right)\right\|-\mathrm{e}^{-\omega s}\left\|u(s)-u\left(t_{0}\right)\right\| \leqq\|z\| \int_{s}^{t} \mathrm{e}^{-\omega \sigma} \mathrm{d} \sigma
$$

for $s, t \in J$ with $s \leqq t$. Hence, we have

$$
\varlimsup_{h \downarrow 0} h^{-1}\left\|u\left(t_{0}+h\right)-u\left(t_{0}\right)\right\| \leqq\|z\|
$$

this is valid for any $z \in \widehat{A} u\left(t_{0}\right)$. Combining this with (4.17), we have

$$
\lim _{h \downarrow 0} h^{-1}\left\|u\left(t_{0}+h\right)-u\left(t_{0}\right)\right\|=\left\|\hat{A} u\left(t_{0}\right)\right\|,
$$


and in particular, $\lim _{h \downarrow 0} h^{-1}\|u(a+h)-u(a)\|=\|\hat{A} u(a)\|$. Thus, applying Theorem 4.4,

$$
\begin{aligned}
\left\|\hat{A} u\left(t_{0}\right)\right\| & =\lim _{h \downarrow 0} h^{-1}\left\|u\left(t_{0}+h\right)-u\left(t_{0}\right)\right\| \\
& \leqq \lim _{h \downarrow 0} \mathrm{e}^{\omega\left(t_{0}-a\right)} h^{-1}\|u(a+h)-u(a)\|=\mathrm{e}^{\omega\left(t_{0}-a\right)}\|\hat{A} u(a)\|,
\end{aligned}
$$

and hence condition (L.5) is obtained. Finally, assume that (L.4) holds. Then by definition, a sequence $\left\{\left(x_{n}, y_{n}\right)\right\} \subset G(\hat{A})$ can be found such that $x_{n} \rightarrow u(a)$ in $X$ and $\left\{y_{n}\right\}$ is bounded in $X^{* *}$. Therefore, Theorem 4.6 implies that

$$
\mathrm{e}^{-2 \omega t}\left\|u(t)-x_{n}\right\|^{2}-\mathrm{e}^{-2 \omega s}\left\|u(s)-x_{n}\right\|^{2} \leqq 2\left\|y_{n}\right\| \int_{s}^{t} \mathrm{e}^{-2 \omega \sigma}\left\|u(\sigma)-x_{n}\right\| \mathrm{d} \sigma
$$

for every $n$ and every pair $s, t \in J$ with $s \leqq t$; hence

$$
\mathrm{e}^{-\omega t}\left\|u(t)-x_{n}\right\|-\mathrm{e}^{-\omega a}\left\|u(a)-x_{n}\right\| \leqq\left\|y_{n}\right\| \int_{a}^{t} \mathrm{e}^{-\omega \sigma} \mathrm{d} \sigma, \quad t \in J, n \geqq 1 .
$$

Now, taking a constant $K>0$ with $\left\|y_{n}\right\| \leqq K$ and letting $n \rightarrow \infty$, we have $\|u(t)-u(a)\| \leqq K \int_{a}^{t} \mathrm{e}^{-\omega \sigma} \mathrm{d} \sigma$ for $t \in J$. From this and Theorem 4.4 it follows that $u(t)$ is Lipschitz continuous on $J$, that is, (L.1) is satisfied. The last Lipschitz condition in the statement of the theorem follows from condition (L.5) and Theorem 4.4.

Q.E.D.

Remark. In view of Remark after Theorem 4.6, we can obtain the same assertion by assuming in Theorem 4.9 that $u(t)$ be an integral solution of (DE) on $J$ such that $u(t) \in \overline{D(A)}$ for $t \in J$. Conditions (L.1) -(L.4) are introduced in Bénilan [2; Chapitre I] as the properties of an integral solution and the crucial step of the proof of Theorem 4.9 is due to him. Also, Theorem 4.9 is closely related to Crandall [8; Corollary 1$]$.

Condition $\left(\mathrm{R}_{\text {loc }} ; A, C\right)$ mentioned in Example 2.14 is a modified version of a condition $\mathrm{S}(A ; C)$ proposed by Bénilan $[1 ; \S 3]$. As a corollary to Theorem 4.9 we can obtain an existence theorem of weak solutions which is closely related to [2; Theorem 1.3$]$ : 
Theorem 4.10. Let $A-\omega$ be dissipative for some $\omega \geqq 0$. Assume that condition $\left(\mathrm{R}_{\mathrm{loc}} ; A, \hat{D}(A)\right)$ holds. Then we have:

(a) For every $x \in \hat{D}(A)$ and $\tau>0$, there exists a unique simple weak solution of (DE) on $[0, \tau]$ with the initial-value $x$.

(b) For every $x \in \overline{D(A)}$ and $\tau>0$, there exists a unique weak solution of $(\mathrm{DE})$ on $[0, \tau]$ with the initial-value $x$.

Proof. (a) Let $x \in \hat{D}(A)$ and $\tau>0$. As already seen in Example 2.15 , there is a positive number $\tau_{1}$ and there is a simple weak solution $u_{1}(t)$ of (DE) on $\left[0, \tau_{1}\right]$ satisfying $u_{1}(0)=x$ together with a Lipschitz condition. Hence, we see from Theorem 4.9 that $\left\|u_{1}(t)-u_{1}(s)\right\| \leqq \mathrm{e}^{\omega \tau_{1}} \mid t-$ $s|\cdot\|\hat{A} x\||$ for $t, s \in\left[0, \tau_{1}\right]$ and $u_{1}\left(\tau_{1}\right) \in \hat{D}(A)$. So, by a routine argument, a positive number $\tau_{2}\left(>\tau_{1}\right)$ exists and (DE) has a simple weak solution $u_{2}(t)$ on $\left[\tau_{1}, \tau_{2}\right]$ such that $u_{2}\left(\tau_{1}\right)=u_{1}\left(\tau_{1}\right)$. Here, we note that $u_{1}\left(\tau_{1}\right) \in$ $D(\hat{A})$ and $\left\|u_{2}(t)-u_{2}(s)\right\| \leqq \mathrm{e}^{\omega\left(\tau_{1}-\tau_{2}\right)}|t-s| \cdot|| \hat{A} u\left(\tau_{1}\right)\left|\left\|\leqq \mathrm{e}^{\omega \tau_{2}}|t-s| \cdot|\|\hat{A} u(0)\||\right.\right.$ for $t, s \in\left[\tau_{1}, \tau_{2}\right]$. In fact, under condition $\left(\mathrm{R}_{\text {loc }} ; A, \hat{D}(A)\right)$, " $x \in \hat{D}(A)$ " is equivalent to " $x \in D(\hat{A})$ " by Theorem 4.9. Now, it is clear that the function $u(t)$, defined on $\left[0, \tau_{2}\right]$ by setting $u(t)=u_{1}(t)$ for $t \in\left[0, \tau_{1}\right]$ and $u(t)=u_{2}(t)$ for $t \in\left[\tau_{1}, \tau_{2}\right]$, gives a simple weak solution of (DE) such that $\|u(t)-u(s)\| \leqq \mathrm{e}^{\omega \tau_{2}}|t-s| \cdot\|\hat{A} u(0)\|$ for $t, s \in\left[0, \tau_{2}\right]$. In this way, there exist a sequence $\left\{\tau_{n}\right\}$ and a sequence of simple weak solutions $u_{n}(t)$ of (DE) on $\left[\tau_{n-1}, \tau_{n}\right]$ such that $u_{n}\left(\tau_{n}\right)=u_{n-1}\left(\tau_{n}\right)$, and a function $u(t)$ obtained by continuing $u_{n}(t), n=1,2, \ldots$ gives a simple weak solution of (DE) on each finite interval $\left[0, \tau_{n_{0}}\right]$ such that $\|u(t)-u(s)\| \leqq \mathrm{e}^{\omega \tau_{n_{0}}} \mid t-$ $s \mid \cdot\|\hat{A} u(0)\|$ for $t, s \in\left[0, \tau_{n_{0}}\right]$. We want to show that we can let $\tau_{n} \rightarrow$ $+\infty$ as $n \rightarrow \infty$. To this end, assume that $\tau_{n} \rightarrow \tau_{+}$for some $\tau_{+}>0$. Then, $u(t)$ is defined on $\left[0, \tau_{+}\right)$and $\|u(t)-u(s)\| \leqq \mathrm{e}^{\omega \tau}|t-s| \cdot\|\hat{A} u(0)\|$ for $t, s \in$ $\left[0, \tau_{+}\right)$. Since $u(t)$ is a weak solution on $\left[0, \tau_{+}\right), u\left(\tau_{+}\right)=\lim _{t \uparrow \tau_{+}} u(t)=$ $\lim _{n \rightarrow \infty} u\left(\tau_{n}\right)$ exists and $u\left(\tau_{+}\right) \in \hat{D}(A)$ (and hence $u\left(\tau_{+}\right) \in D(\hat{A})$ ) by Theorem 4.9. So that there exist sequences $\left\{h_{p}\right\}$ and $\left\{z_{p}\right\}$ and an open ball $B\left(u\left(\tau_{+}\right), \rho\right)$ such that $h_{p} \rightarrow 0, z_{p} \rightarrow 0$ and $\left.\cap \overline{R\left(I-h_{p}\left(A+z_{p}\right)\right.}\right) \supset B\left(u\left(\tau_{+}\right), \rho\right) \cap$ $\hat{D}(A)$. Let $M=\mathrm{e}^{\omega \tau}+\|\hat{A} x\|, \sup _{p}\left\|z_{p}\right\| \leqq 1$ and take a $\tau_{0}>0$ such that $\tau_{0}<$ $\rho / 4\left\{2 \tau_{0}+\exp \left(2 \omega \tau_{0}\right)(M+1)\right\}$. Choose an $n_{0}$ so that $\left|\tau_{n_{0}}-\tau_{+}\right|<\tau_{0} / 2$ and $\left\|u\left(\tau_{n_{0}}\right)-u\left(\tau_{+}\right)\right\|<\rho / 2$. Then, $\bigcap_{p} \overline{R\left(I-h_{p}\left(A+z_{p}\right)\right)} \supset B\left(u\left(\tau_{n_{0}}\right), \rho / 2\right) \cap \hat{D}(A)$, and so, there exists a simple weak solution $u_{n_{0}}(t)$ of (DE) on $\left[\tau_{n_{0}}, \tau_{n_{0}}+\tau_{0}\right]$ 
$\left(\nsupseteq\left[\tau_{n_{0}}, \tau_{+}\right)\right)$. This means that the sequence $\left\{\tau_{n}\right\}$ can be taken such that $\tau_{n} \rightarrow+\infty$. Consequently, we have the assertion (a).

(b) follows from (a) and Corollary 4.8 .

Q.E.D.

Finally, we give some results on the strong solution of (DE).

Theorem 4.11. Let $A-\omega$ be dissipative for some $\omega \geqq 0$ and let $\tilde{A}$ be an extension of $A$ such that $\tilde{A}-\omega$ is maximal dissipative on $D(\hat{A})$. Let $u(t)$ be an $X$-valued continuous function satisfying (4.7) on $J$ and such that $u(t) \in \overline{D(A)}$ for $t \in J$. If $u(t)$ is weakly right-differentiable at a point $t_{0} \in J$ with $t_{0}+h \in J$ for $h>0$ sufficiently small, then $u\left(t_{0}\right) \in$ $D(\tilde{A})$ and $\left(\mathrm{w}-\mathrm{D}^{+}\right) u\left(t_{0}\right) \in \tilde{A} u\left(t_{0}\right)$, where $\left(\mathrm{w}-\mathrm{D}^{+}\right) u\left(t_{0}\right)=\mathrm{w}-\lim _{h \downarrow 0} h^{-1}\left(u\left(t_{0}+\right.\right.$ $\left.h)-u\left(t_{0}\right)\right)$. Therefore, if $u(t)$ is an integral solution of (DE) formulated on $J$ to $A$ and if $u(t)$ is Lipschitz continuous and strongly differentiable almost everywhere on $J$, then $u(t)$ is a strong solution of

$(\mathrm{DE})^{\sim}$

$$
(\mathrm{d} / \mathrm{d} t) u(t) \in \tilde{A} u(t), \quad \text { a.a. } t \in J .
$$

Proof. Let $(x, y) \in G(A)$. Then by the same argument as in the proof of "(L.1) $\rightarrow($ L.2)" part of Theorem 4.9, we have

$$
\begin{aligned}
<h^{-1}\left(u\left(t_{0}+h\right)-u\left(t_{0}\right)\right), w>\leqq h^{-1} \int_{t_{0}}^{t_{0}+h}<y, u(\tau)-x>_{s} \mathrm{~d} \tau & \\
& +\omega h^{-1} \int_{t_{0}}^{t_{0}+h}\|u(\tau)-x\|^{2} \mathrm{~d} \tau
\end{aligned}
$$

for $w \in F\left(u\left(t_{0}\right)-x\right)$ and $h>0$ small. Since $F\left(u\left(t_{0}\right)-x\right)$ is star-weakly compact in $X^{*}$, there exists a $\zeta^{*} \in F\left(u\left(t_{0}\right)-x\right)$ such that $\left\langle y, u\left(t_{0}\right)-x\right\rangle_{s}=$ $\left.<y, \zeta^{*}\right\rangle$. Noting that $<,>_{s}: X \times X \rightarrow R^{1}$ is upper semicontinuous and letting $h \rightarrow+0$, we have

$$
\begin{aligned}
<\left(\mathrm{w}-\mathrm{D}^{+}\right) u\left(t_{0}\right)-y, u\left(t_{0}\right)-x>_{i} & \leqq<\left(\mathrm{w}-\mathrm{D}^{+}\right) u\left(t_{0}\right)-y, \zeta^{*}> \\
& \leqq \omega\left\|u\left(t_{0}\right)-x\right\|^{2}
\end{aligned}
$$

for all $(x, y) \in G(A)$. Now, we set

$$
A_{1} x= \begin{cases}A u\left(t_{0}\right) \cup\left\{\left(\mathrm{w}-\mathrm{D}^{+}\right) u\left(t_{0}\right)\right\} & \text { if } x=u\left(t_{0}\right), \\ A x & \text { if } x \neq u\left(t_{0}\right) .\end{cases}
$$


Then (4.18) states that $A_{1}-\omega$ is a dissipative operator with domain contained in $D(\hat{A})$. Hence $A_{1} \subset \tilde{A}$. Therefore, $u\left(t_{0}\right) \in D(\tilde{A})$ and (w$\left.\mathrm{D}^{+}\right) u\left(t_{0}\right) \in \tilde{A} u\left(t_{0}\right)$.

Q.E.D.

Theorem 4.12. Let $A-\omega$ be dissipative for some $\omega \geqq 0$. Let $u(t)$ be a strong solution of (DE) on an interval $J=[a, b]$, satisfying $u(a)=$ $u_{0} \in \overline{D(A)}$. Let $\left\{h_{p}\right\}$ be any null sequence of positive numbers. Then for every $v>0$, there exists an approximate difference solution $\left\{\left(u_{p, k}^{v}\right.\right.$, $\left.\left.v_{p, k}^{v}\right)\right\}$ of (DS) with error bound $v$ for which condition (S) holds.

Proof. Since $u(t)$ is Lipschitz continuous on $J$, there exists a constant $C>0$ such that $\|u(t)-u(s)\| \leqq C|t-s|$ for $t, s \in J$. Let $h_{p} \rightarrow 0$ and for each $p$, set

$$
w_{p}(t)= \begin{cases}h_{p}^{-1}\left\{u(t)-u\left(t-h_{p}\right)\right\}, & t \in\left[a+h_{p}, b\right] \\ 0 & t \in\left[a, a+h_{p}\right],\end{cases}
$$

and

$$
v(t)=(\mathrm{d} / \mathrm{dt}) u(t) \quad(\in A u(t)) \quad \text { for almost all } t \in J
$$

Since $\left\|w_{p}(t)-v(t)\right\| \rightarrow 0$ as $p \rightarrow \infty$ for almost all $t \in J$, it follows from Egoroff's theorem that for every $v>0$, there exists a closed, measurable set $E_{v}$ in $J$ such that $\mu\left(J \backslash E_{v}\right)<v$ and $\sup _{t \in E_{v}}\left\|w_{p}(t)-v(t)\right\| \rightarrow 0$ as $p \rightarrow \infty$, where $\mu$ denotes the ordinary Lebesgue measure on $R^{1}$. Fix any $v$ with $1>v>0$. Then a $p_{0} \equiv p_{0}(v)$ can be found such that

$$
0<h_{p}<v \text { and }\left\|w_{p}(t)-v(t)\right\| \leqq v \text { for } p \geqq p_{0} \text { and } t \in E_{v} .
$$

Now, we set

$$
u_{p, 0}^{v}=u_{0}, \quad t_{p, 0}=a, \quad \text { for } \quad p \geqq p_{0} .
$$

and then define sequences $\left\{\left(u_{p, k}^{v}, v_{p, k}^{v}\right)\right\} \subset G(A),\left\{v_{p, k}\right\}$ and $\left\{t_{p, k}=t_{p, k-1}+\right.$ $\left.h_{p}+v_{p, k}\right\}$ as follows:

First, define

$$
v_{p, 1}= \begin{cases}0 & \text { if } h_{p}+t_{p, 0} \in E_{v} \\ \operatorname{dis}\left(a+h_{p}, E_{v} \backslash\left[a, a+h_{p}\right)\right) & \text { if } h_{p}+t_{p, 0} \notin E_{v} .\end{cases}
$$


Here, note that $E_{v} \backslash\left[a, a+h_{p}\right)$ is a non-empty closed set if $v$ is sufficiently small. Then $t_{p, 0}+h_{p}+v_{p, 1} \in E_{v}$. We then set

$$
t_{p, 1}=t_{p, 0}+h_{p}+v_{p, 1}, \quad u_{p, 1}^{v}=u\left(t_{p, 1}\right) \text { and } v_{p, 1}^{v}=v\left(t_{p, 1}\right) .
$$

Then, (4.19) implies that

$$
\begin{aligned}
\left\|u_{p, 1}^{v}-h_{p} v_{p, 1}^{v}-u_{p, 0}^{v}\right\| & \leqq\left\|u\left(t_{p, 1}\right)-h_{p} v\left(t_{p, 1}\right)-u\left(a+v_{p, 1}\right)\right\| \\
& +\left\|u\left(a+v_{p, 1}\right)-u_{0}\right\| \leqq h_{p} v+C v_{p, 1} .
\end{aligned}
$$

Assume that $\left(u_{p, l}^{v}, v_{p, l}^{v}\right) \in D(A), v_{p, l}, t_{p, l}, l=1,2, \ldots, k$ are defined as above. If $h_{p}+t_{p, k} \geqq b$, then we set

$$
\left(u_{p, k+1}^{v}, v_{p, k+1}^{v}\right)=\left(u_{p, k}^{v}, v_{p, k}^{v}\right) \text { and } t_{p, k+1}=b .
$$

If $h_{p}+t_{p, k}<b$, then we set

$$
v_{p, k+1}= \begin{cases}0 & \text { if } t_{p, k}+h_{p} \in E_{v}, \\ \operatorname{dis}\left(t_{p, k}+h_{p}, E_{v} \backslash\left[a, t_{p, k}+h_{p}\right)\right) & \text { if } t_{p, k}+h_{p} \notin E_{v},\end{cases}
$$

and

$$
\begin{aligned}
& t_{p, k+1}=t_{p, k}+h_{p}+v_{p, k+1}, \\
& u_{p, k+1}^{v}=u\left(t_{p, k+1}\right), \\
& v_{p, k+1}^{v}=v\left(t_{p, k+1}\right) .
\end{aligned}
$$

In this way, we can define $\left\{\left(u_{p, k}^{v}, v_{p, k}^{v}\right)\right\} \subset G(A),\left\{v_{p, k}\right\}$ and $\left\{t_{p, k}\right\}, k=$ $1,2, \ldots, N_{p}=\left[(b-a) / h_{p}\right]$. Let

$$
\varepsilon_{p, k}^{v}=h_{p}^{-1}\left\{u_{p, k}^{v}-u_{p, k-1}^{v}\right\}-v_{p, k}^{v}, \quad k=1,2, \ldots, N_{p} .
$$

If $t_{p, k-1}+h_{p} \geqq b$, then $h_{p}\left\|\varepsilon_{p, k}^{v}\right\|=h_{p}\left\|v_{p, k-1}^{v}\right\|=h_{p}\left\|v\left(t_{p, k-1}\right)\right\| \leqq C h_{p}$. If $t_{p, k-1}+$ $h_{p}<b$, then

$$
\begin{aligned}
h_{p}\left\|\varepsilon_{p, k}^{v}\right\| & \leqq\left\|u\left(t_{p, k}\right)-u\left(t_{p, k}-h_{p}\right)-h_{p} v\left(t_{p, k}\right)\right\| \\
& \leqq\left\|u\left(t_{p, k}-h_{p}\right)-u\left(t_{p, k-1}\right)\right\| \\
& \leqq h_{p} v+C v_{p, k},
\end{aligned}
$$


and similarly $h_{p}\left\|\varepsilon_{p, k}^{v}\right\| \geqq C v_{p, k}-h_{p} v \geqq C v_{p, k}-h_{p}$.

If $t_{p, k_{0}-1}<b \leqq t_{p, k_{0}}$, then $t_{p, k_{0}-1}=a+\sum_{i=1}^{k_{0}-1}\left(h_{p}+v_{p, i}\right)=a+\left(k_{0}-1\right) h_{p}+$ $\sum_{i=1}^{k_{0}-1} v_{p, i}$ and so $b \leqq t_{p, k_{0}-1}+h_{p}=a+k_{0} h_{p}+\sum_{i=1}^{k_{0}-1} v_{p, i}$. Therefore, setting $N_{p}=$ $\left[(b-a) / h_{p}\right]$, we have $N_{p} h_{p} \leqq b-a \leqq k_{0} h_{p}+v$ and

$$
\sum_{h=k_{0}}^{N_{p}} h_{p}\left\|\varepsilon_{p, k}^{v}\right\| \leqq C h_{p}\left(N_{p}-k_{0}+1\right) \leqq C h_{p}\left(N_{p}-k_{0}\right)+C h_{p} \leqq C v+C h_{p} .
$$

Consequently,

$$
h_{p} \sum_{k=1}^{N_{p}}\left\|\varepsilon_{p, k}^{v}\right\| \leqq v_{p} h_{p}+C \sum_{k=1}^{N_{p}} v_{p, k}+C v+C h_{p} \leqq(b-a+3 C) v .
$$

This means that conditions $\left(\alpha_{1}\right)$ and $\left(\alpha_{2}\right)$ are satisfied for $\left\{\left(u_{p, k}^{v}, v_{p, k}^{v}\right)\right\}$ constructed above. Since $\left\|u_{p, k}^{v}-u_{p, k-1}^{v}\right\|=\left\|u\left(t_{p, k}\right)-u\left(t_{p, k-1}\right)\right\|=\| u\left(t_{p, k-1}+\right.$ $\left.h_{p}+v_{p, k}\right)-u\left(t_{p, k-1}\right) \| \leqq C\left(h_{p}+v_{p, k}\right) \leqq C\left(\left\|\varepsilon_{p, k}^{v}\right\| h_{p}+2 h_{p}\right)$ for $1 \leqq k \leqq N_{p}$ and $p \geqq$ $p_{0}$, condition (S) is also satisfied.

Q.E.D.

Combining the above-mentioned results, we have the following result which is closely related to Crandall-Liggett [6: Theorem II]:

Theorem 4.13. Let $A-\omega$ be maximal dissipative on $D(\hat{A})$ for some $\omega \geqq 0$. Let $u_{0} \in \overline{D(A)}$ and $J=[a, b]$. Then conditions (a) and (b) below on a function $u(t)$ on $J$ are equivalent:

(a) $u(t)$ is a strong solution of (DE) on $J$ with $u(a)=u_{0}$.

(b) For every $v>0$, there exists an approximate difference solution of (DS) (formulated to $A, J$ and $u_{0}$ ) with error bound $v$ for which condition (S) holds, and $u(t)$ is the limit of the approximate difference solutions (in the sense of Theorem 2.8). Moreover $u(t)$ is strongly differentiable almost everywhere on $J$.

Proof. If (a) is satisfied, then (b) follows from Theorem 4.12. Conversely, suppose (b). Then the approximate difference solution converges to a unique simple weak solution $u(t)$ of (DE) by Theorem 2.8. Thus, Theorem 4.6 states that $u(t)$ is an integral solution of (DE) on $J$. Since $u(t)$ is strongly differentiable almost everywhere on $J$ and since $u(t)$ is Lipschitz continuous on $J$ by condition (S), Theorem 4.11 
yields that $u(t)$ becomes a strong solution of (DE) on $J$ and hence condition (a) is satisfied.

Q.E.D.

Corollary 4.14. Let $X$ be reflexive. Let $A-\omega$ be maximal dissipative on $D(\hat{A})$ for some $\omega \geqq 0$. Let $u_{0} \in \overline{D(A)}$ and $J=[a, b]$. Then CP for $A$ and $u_{0}$ has a solution on $J$ if and only if for every $v>0$, there exists an approximate difference solution of (DS) with error bound $v$ for which condition (S) holds. Moreover, in this case $u_{0} \in D(\widehat{A})$.

The proof is obtained by combining Theorems 4.13 and 4.9 with the fact that a Lipschitz continuous function on $J$ is strongly differentiable almost everywhere on $J$, provided that $X$ is reflexive.

Let $z \in X$ and let us consider a differential equation

$$
(\mathrm{d} / \mathrm{dt}) u(t) \in A u(t)+z=(A+z) u(t), \quad t \in[0, \tau] .
$$

We then consider the following type of difference scheme approximating the $(\mathrm{DE} ; z)$ :

$(\mathrm{DS} ; z)$

$$
\left\{\begin{array}{l}
h_{p}^{-1}\left(u_{p, k}-u_{p, k-1}\right)-v_{p, k}-z=\varepsilon_{p, k}, \quad u_{p, 0}=x \\
v_{p, k} \in A u_{p, k}, \quad k=1,2,3, \ldots, h_{p} \downarrow 0 .
\end{array}\right.
$$

Corollary 4.15. Let $X$ be reflexive and let $A$ be dissipative. Then the following conditions are equivalent:

(M.1) $A$ is m-dissipative.

(M.2) For every $x \in \hat{D}(A), z \in X$ and $\tau>0$, there is a strong solution $u(t)$ of $(\mathrm{DE} ; z)$ on $[0, \tau]$ with $u(0)=x$.

(M.3) $D(A)=\hat{D}(A)$ and for every $x \in D(A), z \in X$ and $\tau>0$, there is a strong solution $u(t)$ of $(\mathrm{DE} ; z)$ on $[0, \tau]$ with $u(0)=x$.

(M.4) $A$ is maximal dissipative on $D(\hat{A})$ and for every $x \in D(\hat{A}), z \in$ $X$ and for every $v>0$, there exists an approximate difference solution of $(\mathrm{DS} ; z)$ on $[0, \tau]$ with error bound $v$ for which condition (S) holds.

Proof. It is shown in [12] that (M.1)-(M.3) are equivalent. The equivalency between (M.2) and (M.4) follows from Corollary 4.14.

Q.E.D. 
Corollary 4.15 states that a range condition (R) for $A$ follows from the existence of approximate difference solutions with error bound $v$.

\section{§5. Nonlinear Semigroups of Local Type}

In this section we review the results obtained so far from the viewpoint of the theory of nonlinear semigroups.

Let $A$ be an operator in $X$ such that $A-\omega$ be dissipative for some $\omega \geqq 0$. By WCP for $A$ and $u_{0}$ on a half-real line $R^{+}=[0, \infty)$ we mean a problem to find a strongly continuous function $u(t)$ on $R^{+}$satisfying a given initial condition $u(0)=u_{0}$ such that $u(t)$ restricted to any bounded interval $[0, \tau]$ is a weak solution of $(\mathrm{DE})$ on $[0, \tau]$.

Suppose that there exists a one-parameter family $\{U(t) ; t \geqq 0\}$ of operators from a subset $D$ of $X$ into $X$ such that for every $u_{0} \in D$, $u(t)=U(t) u_{0}$ is a unique solution of WCP for $A$ and $u_{0}$ on $[0, \infty)$. Then we call the operator $U(t)$ a solution operator of WCP for $A$. From the definition of weak solution we see that $D \subset \overline{D(A)}$ and each $U(t)$ maps $D$ into $\overline{D(A)}$. Moreover, Theorem 4.4 states that each $U(t)$ is Lipschitz continuous on $D$ with Lipschitz constant $\mathrm{e}^{\omega t}$. The following result is a nonlinear version of Takahashi-Oharu [24; Theorem 4.1] and at the same time an extension of Miyadera-Oharu [20; Theorem 3]:

Theorem 5.1. Let $A-\omega$ be dissipative. Suppose that there exists a set $D$ such that $\bar{D}=\overline{D(A)}$ and for every $x \in D$, there exists a solution $u(t ; x)$ of WCP for $A$ and $[0, \infty)$ with $u(0, x)=x$. Then there exists a unique semigroup $\{T(t) ; t \geqq 0\}$ of local type on $\overline{D(A)}$ such that $T(t) x=$ $u(t ; x)$ for $x \in D$ and $\{T(t) ; t \geqq 0\}$ forms a semigroup of solution operators of WCP for $A$ on $[0, \infty)$.

Proof. From assumptions, Theorem 4.4 and Corollary 4.8, it follows that for each $x \in \bar{D}=\overline{D(A)}$ there exists a unique weak solution $u(t ; x)$ with $u(0 ; x)=x$. Hence, we can define solution operators $T(t), t \geqq 0$, by setting $T(t) x=u(t ; x)$ for $t \geqq 0$ and $x \in \overline{D(A)}$. We also set $T(0)=$ $I \mid \overline{D(A)}$. Then Theorem 4.4 states that for every pair $x, y \in \overline{D(A)}, \| T(t) x-$ $T(t) y\left\|\leqq \mathrm{e}^{\omega t}\right\| x-y \|$ for all $t \geqq 0$. Now, it remains to prove the semigroup property of $\{T(t) ; t \geqq 0\}$. Let $x \in \overline{D(A)}$ and let $\left\{u_{n}(t ; x)\right\}$ be a sequence 
of $X$-valued, strongly measurable functions satisfying (i)-(iv) in condition (II) and converging to $u(t ; x)$. Let $s \geqq 0$ and set $\tilde{u}_{n}(t ; x)=u_{n}(t+s ; x)$ and $\hat{u}(t ; x)=u(t+s ; x)$. Then $\hat{u}(0 ; x)=u(s ; x)=T(s) x$ and it is clear that conditions (i)-(iii) hold for $\hat{u}_{n}(t ; x)$ and $\hat{u}(t ; x)$ restricted to every bounded interval $[0, \tau]$. Hence, by the unicity of weak solution, $T(t+s) x=$ $u(t+s ; x)$ coincides with $T(t) T(s) x$. This means that $T(t+s)=T(t) T(s)$ on $\overline{D(A)}$ for $t, s \geqq 0$.

Q.E.D.

Combining this theorem with Theorem 4.4 we have the following:

Theorem 5.2. Let $A-\omega$ be dissipative for some $\omega \geqq 0$. Then there is at most one semigroup $\{T(t) ; t \geqq 0\}$ on $\overline{D(A)}$ such that for each $x \in$ $\overline{D(A)}, \quad T(t) x$ is a solution of WCP formulated to A. Moreover, such a semigroup is of local type on $\overline{D(A)}$.

The semigroup $\{T(t) ; t \geqq 0\}$ mentioned in the above theorem will be called a semigroup of solution operators of WCP for $A$. Summarizing the above-mentioned with Theorems 3.9 and 3.10 , we obtain the following:

Theorem 5.3. Let $A-\omega$ be dissipative. Suppose that there exists a set $D \subset X$ with $\bar{D}=\overline{D(A)}$ such that for every $x \in D, \tau>0$ and $v>0$, the approximating difference scheme (DS) has an approximate difference solution $\left\{\left(u_{p, h}^{v}, v_{p, k}^{v}\right)\right\}$ with error bound $v$ satisfying condition (S). Then there exists a unique semigroup $\{T(t)\}$ of local type on $\overline{D(A)}$ such that

(a) for every $\tau>0$ and every null sequence $v_{i} \downarrow 0$, there exists a subsequence $\left\{p_{i}\right\}$ of $\{p\}$ and

$$
T(t) x=\lim _{k h_{p_{i}} \rightarrow t, i \rightarrow \infty} u_{p_{i}, k}^{v_{i}}, \quad t \in[0, \tau], \quad x \in D,
$$

(b) $\{T(t)\}$ is a semigroup of solution operators of WCP for $A$. Moreover, if for every $x \in D$ and $\tau>0$, (DS) has an asymptotically approximate difference solution $\left\{\left(u_{p, k}, v_{p, k}\right)\right\}$ satisfying condition (S)', then we have

(a) for every $\tau>0, T(t) x=\lim _{m h_{p} \rightarrow t, p \rightarrow \infty} u_{p, m}, t \in[0, \tau], x \in D$, instead of (a). 
Proof. Let $x \in D$ and $\tau>0$. Then by Theorem 3.9, $u_{p_{l}, k}^{v_{1}}$ converges to $u(t)$ as $k h_{p_{i}} \rightarrow t$ and $i \rightarrow \infty$ for $t \in[0, \tau]$ and $u(t)$ gives a unique weak solution on $[0, \tau]$. Since $\tau$ is arbitrary, we obtain a unique solution $u(t ; x)$ of WCP for $A$ on $[0, \infty)$. Therefore, by Theorem 5.1, there exists a unique semigroup $\{T(t)\}$ of local type on $\overline{D(A)}$ which is a semigroup of solution operators of WCP for $A$. The last assertion is similarly obtained by employing Theorems 3.10 and 5.1 .

Q.E.D.

Now, in the remainder of this section we restrict ourselves to the case of reflexive Banach spaces and give a characterization of the infinitesimal generator of a semigroup of local type. By the infinitesimal generator of a semigroup $\{T(t) ; t \geqq 0\}$ of local type on a subset $X_{0}$ of $X$ we mean an operator $A_{0}$ which is defined by

$$
A_{0} x=\lim _{h \rightarrow+0} h^{-1}(T(h) x-x)
$$

whenever the limit exists. Note that $A_{0}-\omega$ is dissipative if $\mathrm{e}^{-\omega t} T(t)$ is a contraction on $X_{0}$ for $t \geqq 0$.

Recently, Martin [17] has given a characterization of the infinitesimal generator of a contraction semigroup on $X_{0}$ with $\overline{D\left(A_{0}\right)}=X_{0}$ in the case in which both $X$ and $X^{*}$ are uniformly convex. Miyadera [19] has extended the result to the case in which only $X^{*}$ is uniformly convex. Here we refer to [19].

Definition 5.4. Let $\{T(t) ; t \geqq 0\}$ be a semigroup of local type on $X_{0}$ with the infinitesimal generator $A_{0}$. We define a set $\hat{D}$ by

$$
\hat{D}=\left\{x \in X_{0} ;\|T(h) x-x\|=0(h) \text { as } h \rightarrow+0\right\} .
$$

Let $A$ be an operator in $X$ with domain $D(A)$ contained in $\hat{D}$. If $A$ is an extension of $A_{0}$ (note that $D\left(A_{0}\right) \subset \hat{D}$ ) and if $A-\omega$ is maximal dissipative on $\hat{D}$, then $A$ is called a (g)-operator of $\{T(t) ; t \geqq 0\}$.

If $D\left(A_{0}\right) \neq \emptyset$, then $\hat{D} \supset D\left(A_{0}\right)$ and the $(g)$-operator is well-defined by the maximal principle.

Lemma 5.5. Let $X$ be reflexive and $A$ be an operator in $X$ such 
that $A-\omega$ is maximal dissipative on $D(\hat{A})$ for some $\omega \geqq 0$. Assume that there exists a semigroup $\{T(t) ; t \geqq 0\}$ on $\overline{D(A)}$ of solution operators of WCP for $A$. Then $A=\hat{A}, D(A)=\hat{D}$ and $A$ is a $(g)$-operator of $\{T(t)$; $t \geqq 0\}$.

Proof. Since $\{T(t) ; t \geqq 0\}$ is a semigroup of solution operators of WCP for $A$, Theorem 4.9 yields that $\hat{D}=D(\hat{A})$. Since $X^{* *}=X, \hat{A}$ is an operator in $X$ and at the same time $\hat{A} \supset A$. Let $(x, y) \in G(\hat{A})$ and define an operator $A_{1}$ by putting $A_{1} u=A u \cup\{y\}$ for $u=x$ and $A_{1} u=A u$ otherwise. Then $A_{1}$ is dissipative and $D\left(A_{1}\right) \subset D(\hat{A})$. Since $A$ is maximal dissipative on $\hat{D}=D(\hat{A})$, we have $A_{1} \subset A$; hence $(x, y) \in G\left(A_{1}\right) \subset G(A)$. This means that $G(\hat{A}) \subset G(A)$, that is, $A=\widehat{A}$. Finally, it follows from Theorem 4.11 that $A_{0} \subset A$.

Q.E.D.

Lemma 5.6. Let $X$ be reflexive and $X_{0} \subset X$. Let $\{T(t) ; t \geqq 0\}$ be a semigroup of local type on $X_{0}$ with the infinitesimal generator $A_{0}$ and let $A$ be a (g)-operator of $\{T(t) ; t \geqq 0\}$. Then we have:

(a) Let $X_{1}=\overline{D\left(A_{0}\right)} \cap X_{0}$ and $T_{1}(t) \equiv T(t) \mid X_{1}$ for $t \geqq 0$. If $B$ is an operator in $X$ such that $A_{0} \subset B, D(B) \subset \overline{D\left(A_{0}\right)}$ and $B-\omega$ is dissipative, then $D(B) \subset \hat{D}$ and $\left\{T_{1}(t) ; t \geqq 0\right\}$ is a semigroup of solution operators of WCP for $B$ on $[0, \infty)$. In particular, $\left\{T_{1}(t) ; t \geqq 0\right\}$ is a semigroup of solution operators of WCP for a (g)-operator $A$.

(b) $\overline{D\left(A_{0}\right)} \supset \hat{D}$; and $\overline{D(A)}=X_{0}$ if and only if $\overline{D\left(A_{0}\right)}=X_{0}$.

(c) $A=\hat{A}$ and $D(A)=\hat{D}$.

(d) $A-\omega$ is maximal dissipative on $\overline{D(A)}$.

Proof. (a): If $x \in \hat{D}$, then $T(t) x$ is a strong solution of (DE) on every bounded interval for $A_{0}$ and hence it is a strong solution of (DE) for $B$. Therefore, it follows from Theorem 5.1 that $\left\{T_{1}(t)\right\}$ forms a semigroup of solution operators of WCP for $B$. In view of this and Theorem 4.9 , we see that $D(\hat{B})=\hat{D}$, so $D(B) \subset \hat{D}$. A $(g)$-operator is a special one of those $B$ in question; hence we have the last assertion of (a).

(b) is easily seen from the above-mentioned.

(c) follows from (a) and Lemma 5.5 .

(d); Let $\tilde{A}$ be an extension of $A$ such that $D(\tilde{A}) \subset \overline{D(A)}$ and $\tilde{A}-\omega$ 
is dissipative. Since $\overline{D\left(A_{0}\right)}=\overline{D(A)}$, it follows from (a) that $D(\tilde{A}) \subset \hat{D}$. But, $A-\omega$ is maximal dissipative on $\hat{D}$; hence $\tilde{A} \subset A$. This means that $A-\omega$ is maximal dissipative on $\overline{D(A)}$.

Q.E.D.

Remark. The assertion (d) is due to Miyadera [19; Lemma 2.2].

Definition 5.7. Let $A$ be an operator in $X$. We say that $A$ has the property (D), if for every $x \in D(A), \tau>0$ and every $v$, (DS) formulated on $[0, \tau]$ to $A$ and $x$ has an approximate difference solution $\left\{\left(u_{p, k}^{v}, v_{p, k}^{v}\right)\right\}$ with error bound $v$ for which condition (S) is satisfied.

Using this property, we can characterize a $(g)$-operator as follows:

Theorem 5.8. Let $A$ be an operator in a reflexive Banach space $X$ and let $X_{0}$ be a closed subset of $X$. Then conditions (i)-(iii) below on the operator $A$ are equivalent:

(i) $A$ is a (g)-operator of a semigroup $\{T(t) ; t \geqq 0\}$ of local type on $X_{0}$ such that $X_{0}=\overline{\hat{D}}$.

(ii) $\overline{D(A)}=X_{0}, A$ has the property (D) and $A-\omega$ is maximal dissipative on $X_{0}$.

(iii) $\overline{D(A)}=X_{0}, A-\omega$ is maximal dissipative on $D(\hat{A})$ and there exists a semigroup $\{T(t) ; t \geqq 0\}$ on $\overline{D(A)}$ of solution operators of WCP for $A$.

Moreover, the semigroup in (i) coincides with that in (iii).

Proof. First, assume (i) holds. Since $\hat{D}$ is dense in $X_{0}$, Lemma 5.6 implies that $\overline{D(A)}=X_{0}$ and $A-\omega$ is maximal dissipative on $X_{0}$. Moreover, $D(A)=\hat{D}$ and for every $x \in D(A)$ and $\tau>0, T(t) x$ is a strong solution of $(\mathrm{DE})$ on $[0, \tau]$ for the operator $A$. Therefore, Theorem 4.11 states that for every null sequence $\left\{h_{p}\right\}$ of positive numbers and $v>0$, there exists an approximate difference solution of the (DS) with error bound $v$. This means that $A$ has the property (D) and hence condition (ii) holds. Next, suppose (ii) holds. Then, it follows from Corollary 4.14 that for every $x \in D(A)$ and $\tau>0$, there exist a strong solution of (DE) on $[0, \tau]$ for $A$. Here we note that $A-\omega$ is maximal dissipative on $\left.D(\hat{A})(\subset \overline{D(A})=X_{0}\right)$. Hence, by Theorem 5.1 , there exists a semi- 
group $\{T(t) ; t \geqq 0\}$ of local type on $\overline{D(A)}=X_{0}$ which is also a family of solution operators of WCP for the operator $A$. Hence, (iii) follows.

Finally, it is proved by employing Lemma 5.5 that (i) follows from (iii). The last assertion is clear from the unicity of a weak solution.

Q.E.D.

Miyadera [19] has shown that if $X^{*}$ is uniformly convex, then $A$ is a $(g)$-operator of a semigroup $\{T(t)\}$ of local type on $X_{0}$ such that $\hat{D}$ is dense in $X_{0}$ if and only if $\overline{D(A)}=X_{0}, A$ has the property (G) and $A-\omega$ is maximal dissipative on $X_{0}$. Hence, if $X^{*}$ is uniformly convex

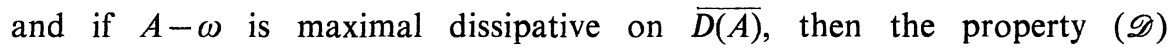
is equivalent to the property $(\mathscr{G})$.

\section{References}

[1] Bénilan, P., Solutions intégrales d'équations d'évolution dans un espace de Banach, C. R. Acad. Sci. Paris 274 (1972), 47-50.

[2] —úquations d'évolution dans un espace de Banach quelconque et applications, Thèse Orsay, Université Paris XI, U. E. R. Mathématique, 91Orsay (France) (1972).

[3] Brezis, H., Équations et inéquations non linéaires dans les espace vectoriels en dualité, Ann. Inst. Fourier, Grenoble, 18, 1 (1968), 115-175.

[4] Brezis, H. and Pazy, A., Accretive sets and differential equations in Banach spaces, Israel J. Math. 8 (1970), 367-383.

[5] —, Convergence and approximation of semigroups of nonlinear operators in Banach spaces, J. Func. Anal. 9 (1972), 63-74.

[6] Crandall, M. and Liggett, T., Generation of semigroups of nonlinear transformations on general Banach spaces, Amer. J. Math. 93 (1971), 265-298.

[7] Crandall, M., The semigroup approach to first order quasilinear equations in several space variables, Israel J. Math. 12 (1972), 108-132.

[8] —, A generalized domain for semigroup generators, Proc. Amer. Math. Soc. 37 (1973), 434-440.

[9] Fujita, H. and Watanabe, S., On the uniqueness and non-uniqueness of solutions of initial-value problems for some quasilinear parabolic equations, C. P. A. M. 21 (1968), 631-652.

[10] Kato, T., Nonlinear evolution equations in Banach spaces, Proc. Symp. Appl. Math. XVII, Amer. Math. Soc., Providence, R. I. (1965), 50-67.

[11] - Nonlinear semigroups and evolution equations, J. Math. Soc. Japan 19 (1967), 503-520.

[12] Kenmochi, N., Remarks on the m-accretiveness of nonlinear operators, Hiroshima Math. J. 3 (1973), 61-68.

[13] Kobayashi, Y., A note on Cauchy problems of semi-linear equations and semi- 
groups in Banach spaces, Proc. Japan Acad. 49 (1973), 514-519.

[14] Kōmura, Y., Nonlinear semigroups in Hilbert space, J. Math. Soc. Japan, 19 (1967), 493-507.

[15] Kōmura, Y., Differentiability of nonlinear semigroups, J. Math. Soc. Japan, 21 (1969), 375-402.

[16] Kružkov, S., First-order quasi-linear equations in several independent variables (in Russian), Math. L'SSR Sbornik 10 (1970) 217-243.

[17] Martin, R. Jr., Generating an evolution system in a class of uniformly convex Banach spaces, J. Func. Anal. 11 (1972), 62-76.

[18] Miyadera, I., Some remarks on semigroups of nonlinear operators, Tôhoku Math. J. 23 (1971), 245-258.

[19] - Generation of semigroups of nonlinear contractions, to appear.

[20] Miyadera, I. and Oharu, S., Approximation of semigroups of nonlinear operators, Tôhoku Math. J. 22 (1970), 24-47.

[21] Oharu, S., On the generation of semigroups of nonlinear contractions, J. Math. Soc. Japan, 22 (4), (1970), 526-550.

[22] Oharu, S. and Takahashi, T., A convergence theorem of nonlinear semigroups and its application to first-order quasilinear equations, to appear in J. Math. Soc. Japan.

[23] Richtmyer, R. and Morton, K., Difference methods for initial-value problems, 2nd. ed., Int. Sci. Publisher, (1967).

[24] Takahashi, T. and Oharu, S., Approximation of operator semigroups in a Banach space, Tôhoku Math. J. 24 (4), (1972), 505-528.

[25] Webb, G., Continuous nonlinear perturbations of linear accretive operators in Banach spaces, J. Func. Anal. 10 (1972), 191-203.

[26] - Nonlinear perturbations of linear accretive operators in Banach spaces, Israel J. Math. 12 (1972), 237-248. 
